Publ. Mat. 59 (2015), 137-233

DOI: 10.5565/PUBLMAT_59115_07

\title{
ON DETERMINANT FUNCTORS AND $K$-THEORY
}

\author{
Fernando Muro, Andrew Tonks, and Malte Witte
}

\begin{abstract}
We extend Deligne's notion of determinant functor to Waldhausen categories and (strongly) triangulated categories. We construct explicit universal determinant functors in each case, whose target is an algebraic model for the 1-type of the corresponding $K$-theory spectrum. As applications, we answer open questions by Maltsiniotis and Neeman on the $K$-theory of (strongly) triangulated categories and a question of Grothendieck to Knudsen on determinant functors. We also prove additivity theorems for low-dimensional $K$-theory of (strongly) triangulated categories and obtain generators and (some) relations for various $K_{1}$-groups. This is achieved via a unified theory of determinant functors which can be applied in further contexts, such as derivators.
\end{abstract}

2010 Mathematics Subject Classification: 19A99, 19B99, 18F25, 18G50, 18G55, 18E10, 18E30.

Key words: Determinant functor, $K$-theory, exact category, Waldhausen category, triangulated category, Grothendieck derivator.

\section{Contents}

Introduction 138

1. Determinant functors 143

1.1. Picard groupoids and categorical groups 143

1.2. For Waldhausen categories 144

1.3. For triangulated categories 149

1.4. Stable quadratic modules 154

1.5. Universal determinant functors 159

1.6. The connection to $K$-theory 166

1.7. Comparison morphisms 167

2. Applications 170

The first and second authors were partially supported by the Spanish Ministry of Economy and Competitiveness under the grants MTM2010-15831 and MTM201342178-P, and by the Government of Catalonia under the grant SGR-119-2009. The first author was also partially supported by the Spanish Ministry of Science and Innovation under a Ramón y Cajal research contract and by the Andalusian Ministry of Economy, Innovation and Science under the grant FQM-5713. 
2.1. Derived and non-derived determinant functors on a Waldhausen category $\quad 170$

2.2. Generators and (some) relations for $K_{1} \quad 171$

2.3. On additivity for low-dimensional $K$-theory of triangulated categories

2.4. Low-dimensional $K$-theory of a triangulated category with a $t$-structure $\quad 177$

2.5. The $K$-theory of some unusual triangulated categories $\quad 186$

2.6. A counterexample to two conjectures by Maltsiniotis 192

3. Strict Picard groupoids 193

3.1. Categorical groups 193

3.2. Crossed modules 195

3.3. Crossed modules and strict categorical groups 196

3.4. Strictifying tensor functors 197

4. A unified approach to determinant functors 201

4.1. Determinant functors for $S_{\text {. }}$-categories 201

4.2. The groupoid of determinant functors 208

4.3. The existence of universal determinant functors 210

4.4. Non-commutative determinant functors 215

4.5. The connection with homotopy theory 219

4.6. Generators and (some) relations for $\pi_{1} \quad 220$

References 229

\section{Introduction}

Determinant functors, considered first by Knudsen and Mumford $[\mathbf{K M}]$, categorify the usual notion of determinant of invertible matrices. The most elementary instance of such a functor sends a finite-dimensional vector space $V$ to the pair

$$
\operatorname{det} V=\left(\operatorname{dim} V, \wedge^{\operatorname{dim} V} V\right) .
$$

The highest exterior power of an automorphism $f: V \cong V$ with matrix $A$ with respect to some basis is multiplication by the determinant, $\wedge^{\operatorname{dim} V} f=\operatorname{det} A$.

Deligne $[\mathbf{D e l}]$ axiomatized the properties of this functor in his definition of determinant functor det: $\mathscr{E} \rightarrow \mathscr{P}$ on an exact category $\mathscr{E}$ with values in a Picard groupoid $\mathscr{P}$. As a functor, det is only defined on isomorphisms, det: iso $(\mathscr{E}) \rightarrow \mathscr{P}$, but short exact sequences $X \mapsto Y \rightarrow C$ 
induce natural isomorphisms

$$
\operatorname{det}(C) \otimes \operatorname{det}(X) \cong \operatorname{det}(Y),
$$

called additivity data, which must satisfy some coherence laws.

Deligne constructed a Picard groupoid of virtual objects $V(\mathscr{E})$ which is the target of a universal determinant functor det: $\mathscr{E} \rightarrow V(\mathscr{E})$, in the sense that any other determinant functor factors through this one in an essentially unique way. The group of isomorphism classes of objects in $V(\mathscr{E})$ is Quillen's $K_{0}(\mathscr{E})$ and the automorphism group of the tensor unit is $K_{1}(\mathscr{E})$. This shows that any interesting exact category has highly non-trivial determinant functors.

Knudsen [Knu1, Knu2] showed by elementary methods that determinant functors on an exact category $\mathscr{E}$ extend to the category of bounded complexes $C^{b}(\mathscr{E})$ in an essentially unique way, generalizing results with Mumford $[\mathbf{K M}]$. This extension is not only defined on isomorphisms, but on quasi-isomorphisms in $C^{b}(\mathscr{E})$.

Quasi-isomorphisms are the weak equivalences of a Waldhausen category structure on $C^{b}(\mathscr{E})$. Therefore, Knudsen's extension theorem hints at the existence of a theory of determinant functors det: $\mathscr{W} \rightarrow \mathscr{P}$ for Waldhausen categories $\mathscr{W}$. This theory is developed in this paper. In particular, we construct a universal determinant functor det: $\mathscr{W} \rightarrow$ $V(\mathscr{W})$ where Waldhausen's $K_{0}(\mathscr{W})$ is the group of isomorphism classes of objects in $V(\mathscr{W})$ and $K_{1}(\mathscr{W})$ is the automorphism group of the tensor unit. Knudsen's results follow then from the Gillet-Waldhausen isomorphism $K_{*}(\mathscr{E}) \cong K_{*}\left(C^{b}(\mathscr{E})\right)$. A theory of determinant functors for Waldhausen categories with values in strict categorical groups has also been developed in [Wit1].

It is a common practice to pass from $C^{b}(\mathscr{E})$ to the derived category $D^{b}(\mathscr{E})$, inverting quasi-isomorphisms. The underlying functor det: we $\left(C^{b}(\mathscr{E})\right) \rightarrow \mathscr{P}$ of a determinant functor det: $C^{b}(\mathscr{E}) \rightarrow \mathscr{P}$ factors uniquely through det: iso $\left(D^{b}\right)(\mathscr{E}) \rightarrow \mathscr{P}$. It would be desirable to enrich this functor with additivity data associated to distinguished triangles $X \rightarrow Y \rightarrow C \rightarrow \Sigma X$ fitting into an appropriate notion of determinant functor for triangulated categories.

Breuning defined recently a notion of determinant functor for triangulated categories. He showed that any triangulated category $\mathscr{T}$ possesses a universal determinant functor det: $\mathscr{T} \rightarrow V\left({ }^{b} \mathscr{T}\right)$ [Bre1], but he did not find a connection between $V\left({ }^{b} \mathscr{T}\right)$ and Neeman's $K$-theories of triangulated categories [Nee2]. Notwithstanding, he proved that if $\mathscr{T}$ has a bounded non-degenerate $t$-structure with heart $\mathscr{A}$, e.g. $\mathscr{T}=D^{b}(\mathscr{A})$, determinant functors on $\mathscr{T}$ essentially coincide with those on the abelian 
category $\mathscr{A}$ in the sense of Deligne [Bre1, Theorem 5.2]. In general, for an exact category $\mathscr{E}$, not all determinant functors det: $\mathscr{E} \rightarrow \mathscr{P}$ extend to a Breuning determinant functor det: $D^{b}(\mathscr{E}) \rightarrow \mathscr{P}$.

In connection with this problem, Grothendieck in a 1973 letter to Knudsen [Knu1, Appendix B] had suggested considering $D^{b}(\mathscr{E})$ as a triangulated category enhanced with a category of 'true triangles', to develop a theory of determinant functors for such enhanced triangulated categories, and to show that any determinant functor det: $\mathscr{E} \rightarrow \mathscr{P}$ extends to det: $D^{b}(\mathscr{E}) \rightarrow \mathscr{P}$ in an essentially unique way for $\mathscr{E}$ additive or abelian. Of course the problem makes sense more generally for $\mathscr{E}$ exact.

We regard the bounded derived category $D^{b}\left(S_{2} \mathscr{E}\right)$ of short exact sequences in $\mathscr{E}$ as the category of true triangles of $D^{b}(\mathscr{E})$. More generally, we work with the homotopy category $\mathrm{Ho}(\mathscr{W})$ of a Waldhausen category $\mathscr{W}$ and the homotopy category $\operatorname{Ho}\left(S_{2} \mathscr{W}\right)$ of cofiber sequences in $\mathscr{W}$. We define derived determinant functors on $\mathscr{W}$ by using only $\operatorname{Ho}(\mathscr{W})$ and $\operatorname{Ho}\left(S_{2} \mathscr{W}\right)$ and we construct a universal derived determinant functor with target $V^{\text {der }}(\mathscr{W})$. The group of isomorphism classes of objects in $V^{\text {der }}(\mathscr{W})$ and the automorphism group of the tensor unit are Garkusha's derived $K$-theory groups $D K_{0}(\mathscr{W})$ and $D K_{1}(\mathscr{W})$ [Gar1], respectively. We then deduce from $[\mathbf{M u r}]$ that derived and non-derived determinant functors on a Waldhausen category $\mathscr{W}$ are essentially the same thing, provided $\mathscr{W}$ has cylinders and a saturated class of weak equivalences. This answers Grothendieck's question in the positive.

Returning to ordinary triangulated categories $\mathscr{T}$, we define new notions of determinant functors whose universal examples compute Neeman's $K$-theories $K_{*}\left({ }^{d} \mathscr{T}\right)$ and $K_{*}\left({ }^{v} \mathscr{T}\right)$ in degrees 0 and 1 [Nee2]. These are functorial $K$-theories, therefore they cannot simultaneously satisfy some desirable properties such as additivity, localization, and agreement with Quillen's $K_{1}$ of exact categories [Sch]. Little is known about these $K$-theories. Neeman asked, for $\mathscr{T}$ a triangulated category with a bounded non-degenerate $t$-structure with heart $\mathscr{A}$, whether $K_{1}(\mathscr{A})=K_{1}\left({ }^{d} \mathscr{T}\right)=K_{1}\left({ }^{v} \mathscr{T}\right)[\mathbf{N e e 2}$, Problem 56]. He did this "in order to show how embarrassingly little we know" (sic) about the $K$-theory of triangulated categories. We here answer affirmatively this question. We also show with an example that $K_{1}\left({ }^{d} \mathscr{T}\right) \neq K_{1}\left({ }^{v} \mathscr{T}\right)$ in general. In addition, we prove that $K_{*}(d \mathscr{T})$ and $K_{*}(v \mathscr{T})$ satisfy additivity in degrees 0 and 1.

We should mention that Neeman has yet another $K$-theory $K_{*}\left({ }^{w} \mathscr{T}\right)$ which is not functorial, and moreover it is only defined for triangulated categories $\mathscr{T}$ with a special kind of algebraic model. This condition is not satisfied by most triangulated categories arising in topology, e.g. 
the stable homotopy category. Neeman managed to show in a series of papers that if $\mathscr{T}$ has the required models and also a $t$-structure as above then $K_{*}(\mathscr{A})=K_{*}\left({ }^{w} \mathscr{T}\right)$ in all dimensions, see [Nee2] and the references therein. Our similarly flavoured theorems in dimension 1 are the first results of this kind for functorial $K$-theories of triangulated categories beyond dimension 0 . Our techniques are completely different to Neeman's.

Another way of enhancing a triangulated category is by considering higher triangles, as suggested by Beĭlinson-Bernstein-Deligne [BBD]. A 2-triangle would be an octahedron, there should be a class of distinguished octahedra satisfying some axioms, generalizing the axioms for distinguished triangles in triangulated categories, and so on. Maltsiniotis [Mal1] worked out an explicit definition and gave these categories the names of strongly triangulated categories or $\infty$-triangulated categories $\mathscr{T}_{\infty}$. He defined a $K$-theory for them, that we denote $K_{*}\left({ }^{s} \mathscr{T}_{\infty}\right)$, and made some conjectures. We consider determinant functors for strongly triangulated categories and show the existence of a universal determinant functor det: $\mathscr{T}_{\infty} \rightarrow V\left({ }^{s} \mathscr{T}_{\infty}\right)$ such that $V\left({ }^{s} \mathscr{T}_{\infty}\right)$ computes $K_{0}\left({ }^{s} \mathscr{T}_{\infty}\right)$ and $K_{1}\left({ }^{s} \mathscr{T}_{\infty}\right)$, as in previous cases. We use this to give an example of an exact category $\mathscr{E}$ for which $K_{1}(\mathscr{E}) \neq K_{1}\left({ }^{s} D^{b}(\mathscr{E})\right)$. This disproves two conjectures due to Maltsiniotis [Mal1, Mal2].

Another application included in this paper is to provide generators and a (possibly incomplete) set of relations for $K_{1}\left({ }^{d} \mathscr{T}\right), K_{1}\left({ }^{s} \mathscr{T}_{\infty}\right)$, and the automorphism group of the tensor unit in $V\left({ }^{b} \mathscr{T}\right)$. This extends results of [Nen, Vak2, MT2].

Our methods are fairly general, and they are suitable for application in other contexts not included in this paper. We consider determinant functors on certain kind of simplicial categories $\mathscr{C}_{\bullet}$ with extra structure, that we call $S_{\bullet}$-categories. Here $S_{\bullet}$ stands for Waldhausen's construction $S_{\bullet} \mathscr{W}\left[\right.$ Wal1], that he used to define $K_{*}(\mathscr{W})$.

Most $K$-theories in the literature can be defined from a certain $S_{\bullet}$-category. We construct a universal determinant functor det: $\mathscr{C}_{\bullet} \rightarrow V\left(\mathscr{C}_{\bullet}\right)$ and show that $V\left(\mathscr{C}_{\bullet}\right)$ computes $\pi_{0}$ and $\pi_{1}$ of a connective spectrum $K\left(\mathscr{C}_{\bullet}\right)$ defined from $\mathscr{C}_{\bullet}$ by using Segal's delooping machine [Seg], in the same way as Waldhausen defined a spectrum $K(\mathscr{W})$ out of $S \bullet \mathscr{W}$ whose homotopy groups are the $K$-theory groups $K_{*}(\mathscr{W})$.

We can apply the general theory to Garkusha's $S \bullet \mathbb{D}$ of a right pointed derivator $\mathbb{D}[$ Gar2]. This yields a definition of determinant functor for derivators det: $\mathbb{D} \rightarrow \mathscr{P}$ that we have not worked out explicitly. Nevertheless, our results show the existence of a universal determinant functor 
det: $\mathbb{D} \rightarrow V(\mathbb{D})$ such that $V(\mathbb{D})$ is a model of the 1-type of GarkushaMaltsiniotis's $K$-theory spectrum $K(\mathbb{D})$ [Gar2, Mal2].

Figure 1 illustrates different types of categories, interpolating between exact and triangulated categories, to which the theory of this paper applies.
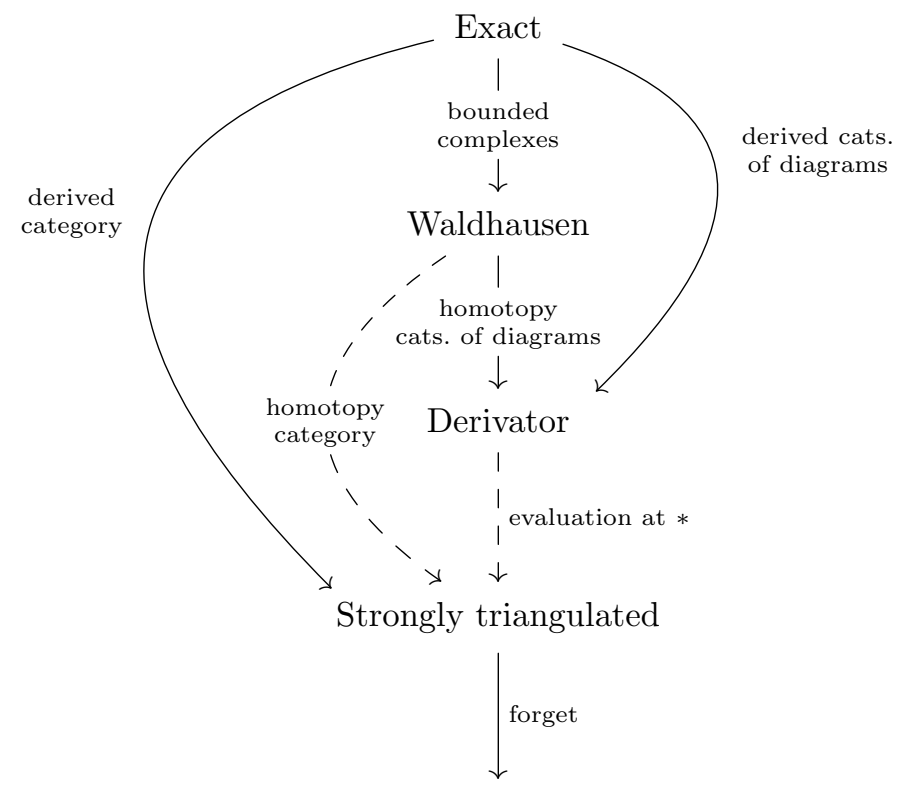

Triangulated

FIgURE 1. The hierarchy between exact and triangulated categories. The dashed arrows indicate that wellknown stability properties are required.

Picard groupoids are algebraic models for spectra with homotopy groups concentrated in dimensions 0 and 1 , and we prove that $V\left(\mathscr{C}_{\bullet}\right)$ is a specific algebraic model of the 1-type of $K\left(\mathscr{C}_{\bullet}\right)$. This strengthens our previous comments on how we obtain low-dimensional $K$-theory groups out of categories of virtual objects. Our explicit models for categories of virtual objects are as strict and small as they can be. They arise from stable quadratic modules, an uncomplicated algebraic structure defined by Baues [Bau] to model stable homotopy types with homotopy groups concentrated in two consecutive degrees. Stable quadratic modules form a 2-category that we prove to be 2-equivalent, in a weak sense, to the 
2-category of Picard groupoids. This is a crucial step in the proof of our main results.

Note that determinant functors on Waldhausen categories have already been successfully applied in non-commutative Iwasawa theory $\left[\right.$ Wit1, Wit2], and in $\mathbb{A}^{1}$-homotopy theory [Eri]. They have also been discussed in the University of Chicago's Geometric Langlands Seminar [Boy], see Remark 1.2.8. Fukaya and Kato give in $[\mathbf{F K}]$ an alternative construction of the category of virtual objects for $\mathscr{E}$ the exact category of projective modules of finite type over a ring $R$.

\section{Determinant functors}

In this section we state our main results: the definition of determinant functors for many kinds of categories, together with the construction of universal determinant functors whose codomains calculate the 1-type of the respective $K$-theory spectra (see Theorems 1.5.4 and 1.6.3). Furthermore, the 1-types of several known comparison maps between these spectra are calculated by straightforward algebraic functors between these codomains. The proofs will be given in Section 4, where we develop a unified approach to these different notions.

1.1. Picard groupoids and categorical groups. Recall that a $\mathrm{Pi}$ card groupoid $\mathscr{P}$ is a symmetric monoidal category [Mac2, VII.1, 7] such that all morphisms are invertible and tensoring with any object $x$ in $\mathscr{P}$ yields an equivalence of categories

$$
x \otimes_{-}: \mathscr{P} \stackrel{\sim}{\longrightarrow} \mathscr{P} .
$$

Some examples are:

- The groupoid Pic $(X)$ of line bundles over a scheme or manifold $X$ with the tensor product over the structure sheaf $\otimes_{\mathscr{O}_{X}}$. If $X=$ Spec $R$ for a commutative ring $R$ then $\operatorname{Pic}(R)=\operatorname{Pic}(X)$ is the groupoid of invertible $R$-modules.

- The category $\mathbf{P i c}^{\mathbb{Z}}(X)$ of graded line bundles over a scheme or manifold $X$. Objects are pairs $(n, L)$ with $L \rightarrow X$ a line bundle and $n: X \rightarrow \mathbb{Z}$ a locally constant map, called degree. Morphisms are only allowed between objects with the same degree. They are simply line bundle isomorphisms. The symmetric monoidal structure is $(n, L) \otimes\left(n^{\prime}, L^{\prime}\right)=\left(n+n^{\prime}, L \otimes_{\mathscr{O}_{X}} L^{\prime}\right)$ with the usual associativity and unit constraints. The graded symmetry constraint is the usual one twisted by a sign depending on the degrees, $(n, L) \otimes\left(n^{\prime}, L^{\prime}\right) \longrightarrow\left(n^{\prime}, L^{\prime}\right) \otimes(n, L): a \otimes b \longmapsto(-1)^{n \cdot n^{\prime}} b \otimes a$. 
Picard groupoids are also called symmetric categorical groups. A categorical group is a monoidal groupoid such that $x \otimes_{\text {- }}$ is an equivalence of categories for any object $x$.

Associativity and commutativity (symmetry) isomorphisms in these monoidal groupoids will simply be denoted by

$$
\text { ass }: x \otimes(y \otimes z) \longrightarrow(x \otimes y) \otimes z, \quad \text { comm: } x \otimes y \longrightarrow y \otimes x .
$$

Recall also that a tensor functor $F$ is equipped with natural multiplication isomorphisms

$$
\text { mult: } F(x) \otimes F(y) \longrightarrow F(x \otimes y) .
$$

See Section 3 for further details.

1.2. For Waldhausen categories. A Waldhausen category $\mathscr{W}$ is a category together with a distinguished zero object 0 and two subcategories $\operatorname{cof}(\mathscr{W})$ and we $(\mathscr{W})$ containing iso $(\mathscr{W})$, whose morphisms are called cofibrations $\rightarrow$ and weak equivalences $\stackrel{\sim}{\rightarrow}$, respectively. Some axioms must be satisfied, in particular the pushout of any map and a cofibration $Y \leftarrow X \longmapsto Z$ exists in $\mathscr{W}$, and is denoted $Y \cup_{X} Z$. These categories were introduced by Waldhausen [Wal2, Section 1.2], under the name of categories with cofibrations and weak equivalences, as a general setting where a reasonable $K$-theory can be defined extending Quillen's [Qui].

Example 1.2.1. The following are three simple examples of Waldhausen categories:

- An exact category $\mathscr{E}$ is a full additive subcategory of an abelian category closed under extensions. A short exact sequence in $\mathscr{E}$ is a short exact sequence in the ambient abelian category between objects in $\mathscr{E}$. The first arrow of a short exact sequence in $\mathscr{E}$ is called an admissible monomorphism. Admissible monomorphisms are the cofibrations of a Waldhausen category structure on $\mathscr{E}$ where weak equivalences are isomorphisms we $(\mathscr{E})=\operatorname{iso}(\mathscr{E})$. One must also choose a zero object 0 in $\mathscr{E}$. Examples of exact categories are abelian categories, the category $\operatorname{proj}(R)$ of finitely generated projective modules over a ring $R$, and the category $\operatorname{vect}(X)$ of vector bundles of finite rank over a scheme or a manifold $X$.

- The category $C^{b}(\mathscr{E})$ of bounded complexes in an exact category $\mathscr{E}$. Cofibrations are levelwise split monomorphisms and weak equivalences are quasi-isomorphisms, i.e. chain morphisms inducing isomorphisms in homology computed in the ambient abelian category. The distinguished zero object is the complex with 0 everywhere. 
- The category $C^{b}(\mathscr{E})$ with the same weak equivalences and distinguished zero object as above, but levelwise admissible monomorphisms as cofibrations. This Waldhausen category has the same $K$-theory as the previous one. We will always assume that $C^{b}(\mathscr{E})$ is endowed with this Waldhausen category structure so that the inclusion of complexes concentrated in degree $0, \mathscr{E} \subset C^{b}(\mathscr{E})$, preserves all the structure.

Coproducts $X \sqcup Y=X \cup_{0} Y$ exist in $\mathscr{W}$. Also, for any cofibration $f: X \longmapsto Y$ we have a cofiber sequence

$$
\Delta: X \stackrel{f}{\longrightarrow} Y \longrightarrow C^{f} .
$$

Here $C^{f}=0 \cup_{X} Y$ is the cofiber, sometimes denoted by $Y / X$, and the second morphism is the canonical map to the push-out. Cofiber sequences in exact categories are short exact sequences.

The following definition of determinant functor generalizes Deligne's definition for the special case of exact categories [Del, 4.2].

Definition 1.2.3. Let $\mathscr{W}$ be a Waldhausen category and $\mathscr{P}$ a Picard groupoid. A determinant functor det: $\mathscr{W} \rightarrow \mathscr{P}$ consists of a functor from the subcategory of weak equivalences,

$$
\operatorname{det}: \text { we }(\mathscr{W}) \longrightarrow \mathscr{P},
$$

together with additivity data: for any cofiber sequence $\Delta$ as above, a morphism

$$
\operatorname{det}(\Delta): \operatorname{det}\left(C^{f}\right) \otimes \operatorname{det}(X) \longrightarrow \operatorname{det}(Y),
$$

natural with respect to weak equivalences of cofiber sequences, given by commutative diagrams in $\mathscr{W}$,

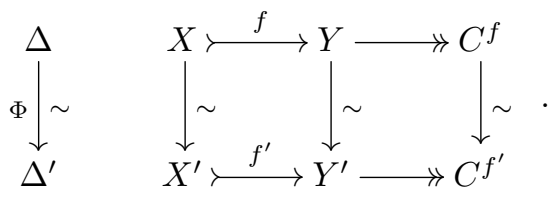


The following two axioms must be satisfied.

(1) Associativity: given a staircase commutative diagram

$\Theta$ :

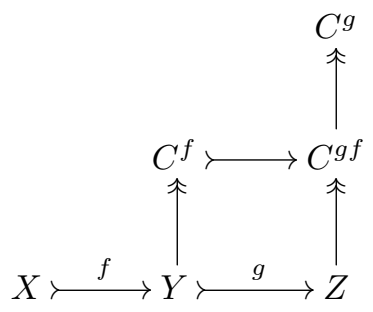

containing four cofiber sequences in $\mathscr{W}$,

$$
\begin{aligned}
& \Delta_{f}: X \stackrel{f}{\longrightarrow} Y \longrightarrow C^{f}, \quad \Delta_{g}: Y \stackrel{g}{\longrightarrow} Z \longrightarrow C^{g}, \\
& \Delta_{g f}: X \stackrel{g f}{\longrightarrow} Z \longrightarrow C^{g f}, \quad \widetilde{\Delta}: C^{f} \longleftrightarrow C^{g f} \longrightarrow C^{g},
\end{aligned}
$$

the following diagram in $\mathscr{P}$ commutes:

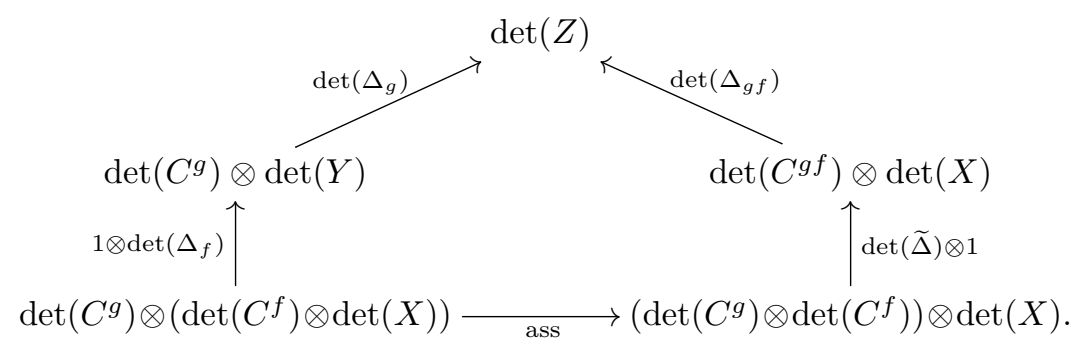

(2) Commutativity: given two objects $X$ and $Y$ in $\mathscr{W}$, there are two cofiber sequences associated to the inclusions and projections of the two factors of their coproduct,

$$
\Delta_{1}: X \succ X \sqcup Y \longrightarrow Y, \quad \Delta_{2}: Y \succ X \sqcup Y \longrightarrow X,
$$

and the following triangle commutes:

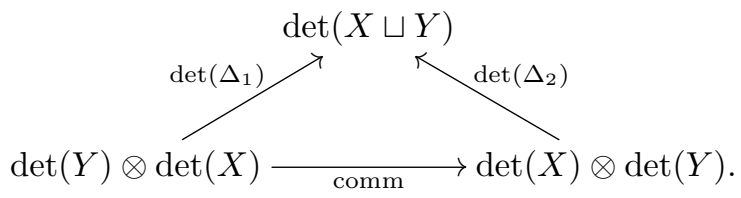


If $\mathscr{P}$ is just a categorical group, we define non-commutative determinant functors det: $\mathscr{W} \rightarrow \mathscr{P}$ as above, but omitting the commutativity axiom.

The notation det: $\mathscr{W} \rightarrow \mathscr{P}$ may seem to be misleading at a first glance. It suggests that det is defined on all morphisms of $\mathscr{W}$. As a functor, it is only defined on weak equivalences. Nevertheless, it also takes values on cofiber sequences in the form of additivity data. This justifies the usual notation.

Example 1.2.7. The prototypical example of determinant functor on an exact category is the following. Suppose $X$ is a scheme or manifold. Then the rank of a vector bundle $E$ over $X$ is a locally constant function rk $E: X \rightarrow \mathbb{Z}$, and we can define a determinant functor $\operatorname{det}: \operatorname{vect}(X) \rightarrow$ $\mathbf{P i c}^{\mathbb{Z}}(X)$ as follows:

$$
\operatorname{det}(E)=\left(\mathrm{rk} E, \wedge_{\mathscr{O}_{X}}^{\mathrm{rk} E} E\right) .
$$

As a particular case, we get a determinant functor $\operatorname{det}: \operatorname{proj}(R) \rightarrow$ $\mathbf{P i c}^{\mathbb{Z}}(R)$.

Knudsen-Mumford $[\mathbf{K M}]$ showed that this example can be extended to bounded complexes $\operatorname{det}: C^{b}(\operatorname{vect}(X)) \rightarrow \mathbf{P} \mathbf{~ i c}^{\mathbb{Z}}(X)$ in an essentially unique way. Knudsen [Knu1] generalized this result to arbitrary determinant functors on an exact category. These results are proved by a lengthy direct computation. We here derive them from the existence of universal determinant functors with values in a Picard groupoid computing the first two $K$-theory groups and from the Gillet-Waldhausen theorem.

Remark 1.2.8. In the seminar notes [Boy] a tentative definition of determinant functor for Waldhausen categories is given. Drinfeld wonders whether this notion is such that a universal determinant functor exists and whether the target is associated to Waldhausen's $K$-theory [Boy, Endnote 7)]. The results on non-commutative determinant functors in [Wit1, Section 2.3] and in Subsection 4.4 of this paper show that the answer is yes provided we introduce a slight correction in [Boy, (ii) in Section 2]: we must require the induced map $A \cup_{A^{\prime}} B^{\prime} \rightarrow B$ to be a cofibration, compare [MT2, Proposition 1.6]. The same correction must be made in [Eri, Definition 2.2.1 (c)].

Definition 1.2.9. A determinant functor det: $\mathscr{W} \rightarrow V(\mathscr{W})$ is universal if any determinant functor $\operatorname{det}^{\prime}: \mathscr{W} \rightarrow \mathscr{P}$ factors through det in an 
essentially unique way. More precisely, there exists a diagram

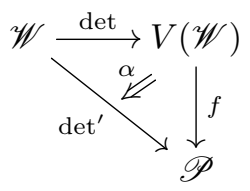

where $f$ is a symmetric tensor functor and $\alpha$ is a natural transformation compatible with the additivity data, i.e. for any cofiber sequence $\Delta$ as above, the following square commutes:

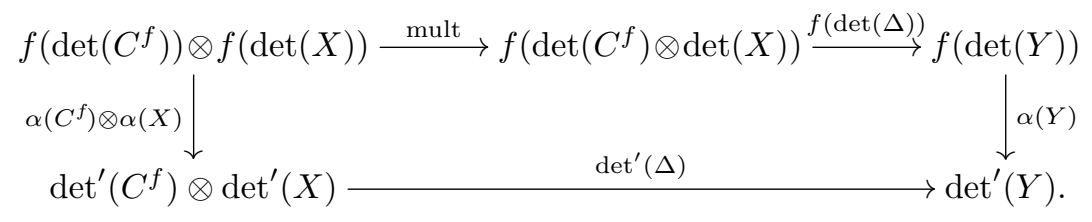

Moreover, if

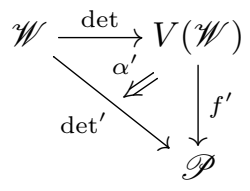

is another such factorization, then there exists a unique tensor natural transformation $\beta: f \Rightarrow f^{\prime}$ such that (1.2.10) coincides with the pasting of

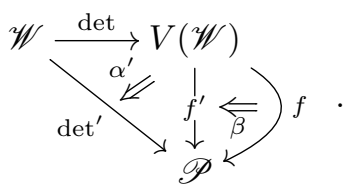

We call $V(\mathscr{W})$ the category of virtual objects of $\mathscr{W}$, following Deligne's terminology for exact categories. This Picard groupoid is well defined up to equivalence. We later show its existence, producing a very explicit model.

Universal non-commutative determinant functors are defined in the obvious way, dropping the symmetry condition from the tensor functors $f$ and $f^{\prime}$.

Any Waldhausen category $\mathscr{W}$ has an associated homotopy category $\mathrm{Ho}(\mathscr{W})$ obtained by formally inverting weak equivalences in $\mathscr{W}$. We can also consider the Waldhausen category $S_{2} \mathscr{W}$ of cofiber sequences in $\mathscr{W}[\mathbf{W a l 2}]$. 
Definition 1.2.11. Let $\mathscr{P}$ be a Picard groupoid. A derived determinant functor det: $\mathscr{W} \rightarrow \mathscr{P}$ consists of a functor from the category of isomorphisms in the homotopy category,

$$
\text { det: } \operatorname{iso}(\operatorname{Ho}(\mathscr{W})) \longrightarrow \mathscr{P},
$$

together with additivity data: for any cofiber sequence $\Delta: X \longmapsto Y \rightarrow C^{f}$ in $\mathscr{W}$, a morphism in $\mathscr{P}$

$$
\operatorname{det}(\Delta): \operatorname{det}\left(C^{f}\right) \otimes \operatorname{det}(X) \longrightarrow \operatorname{det}(Y),
$$

natural in $\operatorname{Ho}\left(S_{2} \mathscr{W}\right)$. Axioms (1) and (2) in Definition 1.2.3 must be satisfied.

Non-commutative derived determinant functors with target a categorical group $\mathscr{P}$ are defined by removing the commutativity axiom (2). Moreover, universal (non-commutative) derived determinant functors are defined as in Definition 1.2.9.

Derived determinant functors are related to Grothendieck's question to Knudsen that we answer positively in Subsection 2.1.

1.3. For triangulated categories. A triangulated category $\mathscr{T}$ is an additive category together with an equivalence $\Sigma: \mathscr{T} \stackrel{\sim}{\rightarrow} \mathscr{T}$ and a class of diagrams called distinguished triangles

$$
\Delta: X \stackrel{f}{\longrightarrow} Y \stackrel{i^{f}}{\longrightarrow} C^{f} \stackrel{q^{f}}{\longrightarrow} \Sigma X,
$$

also depicted as

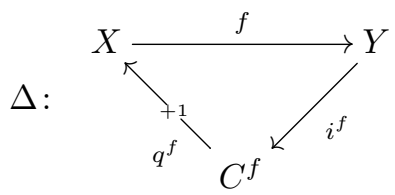

where $X \stackrel{+1}{\rightarrow} Y$ denotes a morphism $X \rightarrow \Sigma Y$. Any diagram like (1.3.1) where two consecutive morphisms compose to 0 will be called a triangle. We say that $f$ is the base of the triangle.

Distinguished triangles must satisfy a set of well-known axioms, see [Nee1]. Verdier's octahedral axiom says that given composable morphisms

$$
X \stackrel{f}{\longrightarrow} Y \stackrel{g}{\longrightarrow} Z,
$$

and three distinguished triangles $\Delta_{f}, \Delta_{g}$, and $\Delta_{g f}$ with bases $f, g$, and $g f$, respectively, then there exists a diagram with the shape of an 
octahedron

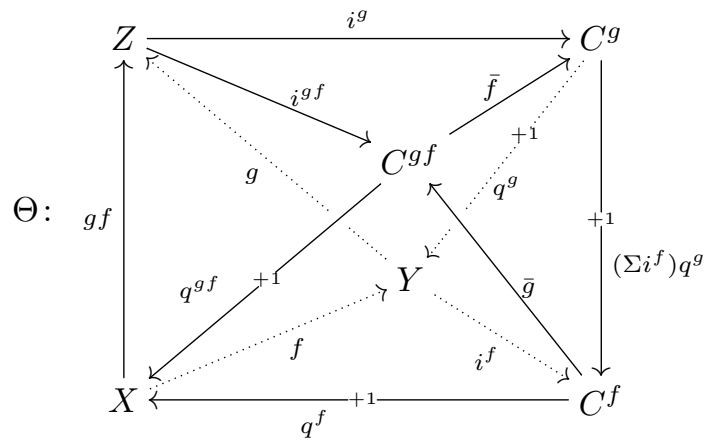

in which three faces are $\Delta_{f}, \Delta_{g}$, and $\Delta_{g f}$, four faces are commutative triangles, and the remaining face

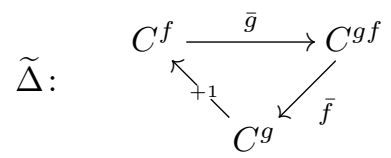

is also a distinguished triangle. Moreover, three planes divide the octahedron into two square pyramids. The squares perpendicular to the page must be commutative.

Verdier's axiom is about the existence of appropriate $\bar{f}$ and $\bar{g}$; the rest is given. Any diagram with the properties of (1.3.2) will be called an octahedron.

A special octahedron is an octahedron (1.3.2) such that the two commutative squares are homotopy cartesian in the sense of [Nee1, Definition 1.4.1], i.e. the following triangles are distinguished:

$$
\begin{gathered}
Y \stackrel{\left(\begin{array}{c}
g \\
-i^{f}
\end{array}\right)}{\longrightarrow} Z \oplus C^{f} \stackrel{\left(i^{g f}, \bar{g}\right)}{\longrightarrow} C^{g f} \stackrel{q^{g} \bar{f}}{\longrightarrow} \Sigma Y, \\
C^{g f} \stackrel{\left(\begin{array}{c}
q^{g f} \\
-\bar{f}
\end{array}\right)}{\longrightarrow} \Sigma X \oplus C^{g} \stackrel{\left(\Sigma f, q^{g}\right)}{\longrightarrow} \Sigma Y \stackrel{\Sigma\left(\bar{g} q^{f}\right)}{\longrightarrow} \Sigma C^{g f} .
\end{gathered}
$$

Remark 1.3.4. Special octahedra where first introduced by BeullinsonBernstein-Deligne [BBD, Remarque 1.1.13]. If $\mathscr{T}$ is a derived category, or more generally a stable homotopy category, then it is well known that the standard octahedral completion of two composable morphisms $X \rightarrow Y \rightarrow Z$ is special in this sense. In general, the octahedral axiom completion can be chosen so that one of the two triangles in (1.3.3) is distinguished, compare [Nee1, Proposition 1.4.6]. In particular, if the completion happens to be unique then the resulting octahedron is special. This observation will be useful in applications concerning $t$-structures. 
Definition 1.3.5. Let $\mathscr{P}$ be a Picard groupoid. A Breuning determinant functor det: $\mathscr{T} \rightarrow \mathscr{P}$ consists of a functor

$$
\operatorname{det}: \operatorname{iso}(\mathscr{T}) \longrightarrow \mathscr{P},
$$

together with additivity data: for any distinguished triangle $\Delta$ as in (1.3.1), a morphism in $\mathscr{P}$

$$
\operatorname{det}(\Delta): \operatorname{det}\left(C^{f}\right) \otimes \operatorname{det}(X) \longrightarrow \operatorname{det}(Y),
$$

natural with respect to distinguished triangle isomorphisms

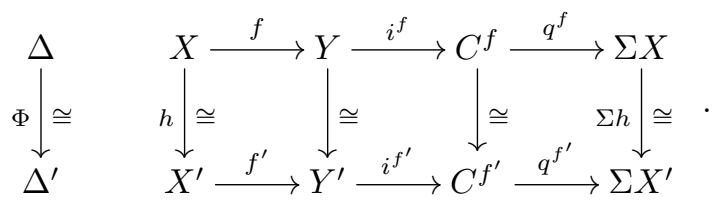

The following two axioms must be satisfied, see [Bre1, Definition 3.1].

(1) Associativity: for any octahedron $\Theta$ as in (1.3.2) the following diagram in $\mathscr{P}$ commutes:

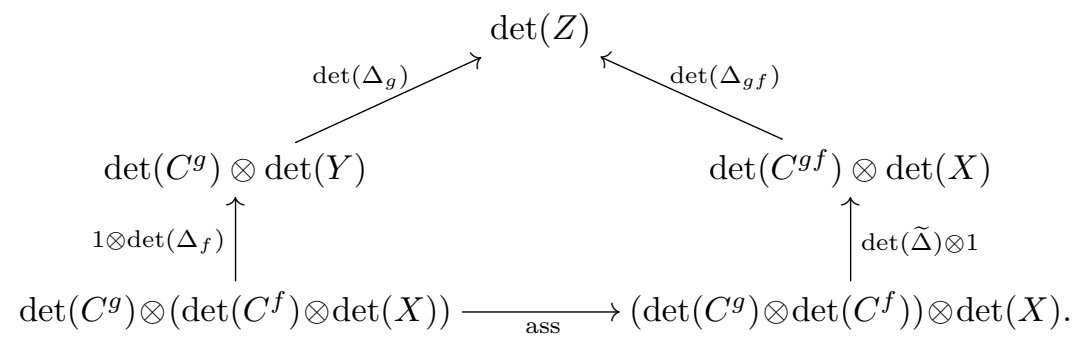

(2) Commutativity: given two objects $X, Y$ in $\mathscr{T}$, if we consider the two distinguished triangles associated to the inclusions and projections of the two factors of their coproduct

$$
\begin{aligned}
& \Delta_{1}: X \longrightarrow X \oplus Y \longrightarrow Y \stackrel{0}{\longrightarrow} \Sigma X, \\
& \Delta_{2}: Y \longrightarrow X \oplus Y \longrightarrow X \stackrel{0}{\longrightarrow} \Sigma Y,
\end{aligned}
$$

then the following diagram commutes:

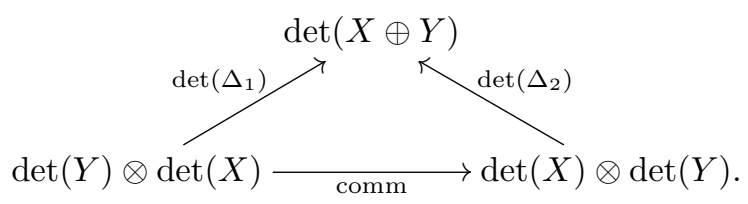


A special determinant functor is defined in the same way, but we only require associativity with respect to special octahedra.

We can define non-commutative Breuning or special determinant functors allowing $\mathscr{P}$ to be any categorical group and dropping the commutativity axiom. We can also define universal (non-commutative) Breuning or special determinant functors as in Definition 1.2.9. The only difference is that $\Delta$ must be a distinguished triangle instead of a cofiber sequence.

We now recall Vaknin's notion of virtual triangle [Vak3]. A contractible triangle is a direct sum of triangles of the form

$$
\begin{gathered}
A \longrightarrow A \longrightarrow 0 \longrightarrow \Sigma A, \\
0 \longrightarrow B \longrightarrow \text { \ } B \longrightarrow 0, \\
C \longrightarrow 0 \longrightarrow \stackrel{1}{\longrightarrow} \Sigma C,
\end{gathered}
$$

i.e.

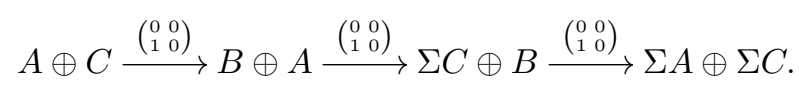

Contractible triangles are always distinguished.

The definition of virtual triangle is a little bit involved. As a special case we have the triangles

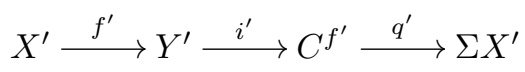

such that there exist distinguished triangles as follows:

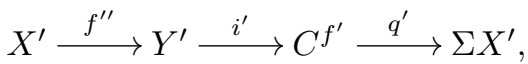

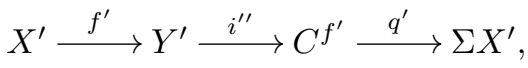

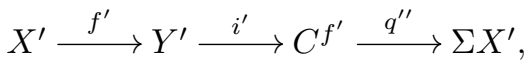

i.e. we can replace each arrow in $X^{\prime} \stackrel{f^{\prime}}{\rightarrow} Y^{\prime} \stackrel{i^{\prime}}{\rightarrow} C^{f^{\prime}} \stackrel{q^{\prime}}{\rightarrow} \Sigma X^{\prime}$ so as to obtain a distinguished triangle.

In general, a triangle $X \stackrel{f}{\rightarrow} Y \stackrel{i}{\rightarrow} C^{f} \stackrel{q}{\rightarrow} \Sigma X$ is a virtual triangle if the direct sum with some contractible triangle gives the special case of 
virtual triangle above,

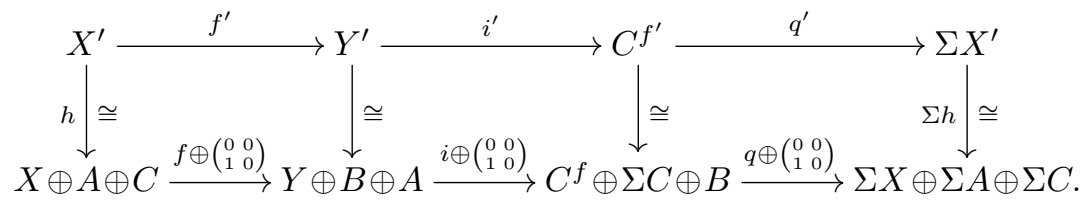

A virtual octahedron is a diagram $\Theta$ as in (1.3.2) where four faces $\Delta_{f}, \Delta_{g}, \Delta_{g f}, \widetilde{\Delta}$ are virtual triangles, the remaining four faces are commutative triangles, and we have two commutative squares as in classical octahedra.

Remark 1.3.8. In a virtual octahedron, the triangles (1.3.3) are always virtual by Vaknin's two-out-of three property [Vak3, Section 1.3] applied to
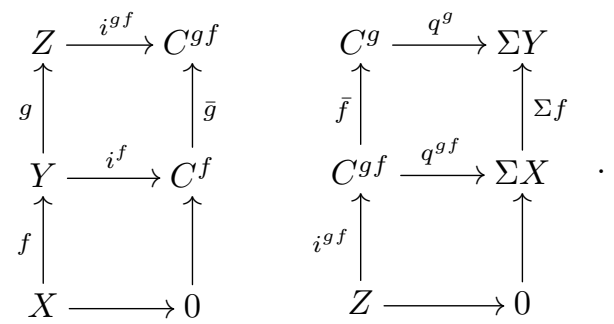

Definition 1.3.9. A virtual determinant functor det: $\mathscr{T} \rightarrow \mathscr{P}$ is a functor

$$
\operatorname{det}: \operatorname{iso}(\mathscr{T}) \longrightarrow \mathscr{P}
$$

together with additivity data: for any virtual triangle $\Delta$ as in (1.3.1), a morphism

$$
\operatorname{det}(\Delta): \operatorname{det}\left(C^{f}\right) \otimes \operatorname{det}(X) \longrightarrow \operatorname{det}(Y),
$$

natural with respect to virtual triangle isomorphisms. In addition we require associativity for virtual octahedra and commutativity as in Definition 1.3.5.

We can also define (universal, non-commutative) virtual determinant functors, compare Definition 1.3.5.

Following a remark of Ber̆linson-Bernstein-Deligne [BBD, 1.1.14], Maltsiniotis defined the notion of strongly triangulated category $\mathscr{T}_{\infty}$, also termed $\infty$-triangulated category [Mal1]. He indicated how the bounded derived category $D^{b}(\mathscr{E})$ can be endowed with such a structure. He also defined truncated versions, called $n$-pretriangulated category. A 3-pretriangulated category $\mathscr{T}_{3}$ is a triangulated category together with a family of distinguished octahedra (3-triangles in Maltsiniotis's terminology), 
which must satisfy some axioms generalizing the axioms for distinguished triangles in a triangulated category, see [Mal1, 1.3 and 1.4].

Definition 1.3.10. Let $\mathscr{P}$ be a Picard groupoid. A determinant functor det: $\mathscr{T}_{3} \rightarrow \mathscr{P}$ is the same as a determinant functor on the underlying triangulated category, except that we only require the associativity axiom (1) to hold for distinguished octahedra. A determinant functor on a strongly triangulated category is a determinant functor on the underlying 3-pretriangulated category. We similarly define (universal, non-commutative) determinant functors in this context.

1.4. Stable quadratic modules. In this section we introduce algebraic tools which allow a very explicit construction of universal determinant functors. The main tools are Baues's stable quadratic modules $[\mathbf{B a u}]$.

Definition 1.4.1. A stable quadratic module $C_{*}$ consists of group homomorphisms

$$
C_{0}^{a b} \otimes C_{0}^{a b} \stackrel{\langle\cdot, \cdot\rangle}{\longrightarrow} C_{1} \stackrel{\partial}{\longrightarrow} C_{0},
$$

satisfying the following equations, $c_{i}, c_{i}^{\prime} \in C_{i}$ :

(1) $\left\langle c_{0}, c_{0}^{\prime}\right\rangle+\left\langle c_{0}^{\prime}, c_{0}\right\rangle=0$,

(2) $\partial\left\langle c_{0}, c_{0}^{\prime}\right\rangle=\left[c_{0}^{\prime}, c_{0}\right]$

(3) $\left\langle\partial\left(c_{1}\right), \partial\left(c_{1}^{\prime}\right)\right\rangle=\left[c_{1}^{\prime}, c_{1}\right]$.

Here $[x, y]=-x-y+x+y$ denotes the commutator of two elements $x, y$ in a group. We will denote group laws additively, although the groups may be non-abelian. We write $C_{0}^{a b}$ for the abelianization of $C_{0}$, and use the same notation for elements of $C_{0}$ as for their images in $C_{0}^{a b}$ since in context there is no ambiguity.

A morphism of stable quadratic modules $f: C_{*} \rightarrow D_{*}$ consists of group homomorphisms $f_{i}: C_{i} \rightarrow D_{i}, i=0,1$, satisfying $\partial f_{1}=f_{0} \partial$ and $\left\langle f_{0}, f_{0}\right\rangle=f_{1}\langle\cdot, \cdot\rangle$.

A homotopy $\alpha: f \Rightarrow g$ between two morphisms $f, g: C_{*} \rightarrow D_{*}$ is a function $\alpha: C_{0} \rightarrow D_{1}$ such that

$$
\begin{aligned}
\alpha\left(c_{0}+c_{0}^{\prime}\right) & =\alpha\left(c_{0}\right)^{g_{0}\left(c_{0}^{\prime}\right)}+\alpha\left(c_{0}^{\prime}\right), \\
\partial \alpha\left(c_{0}\right) & =-g_{0}\left(c_{0}\right)+f_{0}\left(c_{0}\right), \\
\alpha \partial\left(c_{1}\right) & =-g_{1}\left(c_{1}\right)+f_{1}\left(c_{1}\right) .
\end{aligned}
$$

Here the exponential notation $d_{1}^{d_{0}}$ stands for $d_{1}+\left\langle d_{0}, \partial\left(d_{1}\right)\right\rangle$. We follow the conventions in [Wit1], which are opposite to [Bau, MT1, MT2]. 
Stable quadratic modules, morphisms and homotopies form a 2-category. Horizontal composition is given by composition of maps, and the vertical composition of two homotopies

$$
f \stackrel{\alpha}{\Longrightarrow} g \stackrel{\beta}{\Longrightarrow} h
$$

is given by the map $\beta+\alpha$, compare [BM, Proposition 7.2].

Notice that, if we think of a stable quadratic module $C_{*}$ as a nonabelian chain complex concentrated in degrees 0 and 1 ,

$$
\cdots \longrightarrow 0 \longrightarrow C_{1} \stackrel{\partial}{\longrightarrow} C_{0} \longrightarrow 0 \longrightarrow \cdots,
$$

enriched with the bracket operation, the homotopies above are analogs of classical chain homotopies.

Remark 1.4.2. The bracket $\langle\cdot, \cdot\rangle$ behaves as a bilinear form, since its source is the tensor square of the abelianization of $C_{0}$. It follows that the groups $C_{0}$ and $C_{1}$ have nilpotency class 2. Groups of nilpotency class 2 are very close to abelian groups. Commutators need not vanish, but they are central, and the commutator bracket $[\cdot, \cdot]$ behaves as a bilinear form: it factors through the tensor square of the abelianization. The bracket $\langle\cdot, \cdot\rangle$ also maps to the center of $C_{1}$. Moreover, $\partial\left(C_{1}\right) \subset C_{0}$ is normal.

The group $C_{0}$ acts on the right of $C_{1}$ by the formula

$$
c_{1}^{c_{0}}=c_{1}+\left\langle c_{0}, \partial\left(c_{1}\right)\right\rangle .
$$

The exponent of $c_{1}^{c_{0}}$ is actually in the abelianization $C_{0}^{a b}$. Moreover, we have

$$
c_{1}+c_{1}^{\prime}=c_{1}^{\prime}+c_{1}^{\partial\left(c_{1}^{\prime}\right)}=\left(c_{1}^{\prime}\right)^{-\partial\left(c_{1}\right)}+c_{1} .
$$

Homotopies satisfy $\alpha(0)=\alpha(0+0)=\alpha(0)^{g_{0}(0)}+\alpha(0)$, hence

$$
\alpha(0)=0 .
$$

Moreover, $0=\alpha(0)=\alpha\left(c_{0}-c_{0}\right)=\alpha\left(c_{0}\right)^{-g_{0}\left(c_{0}\right)}+\alpha\left(-c_{0}\right)$, therefore

$$
\alpha\left(-c_{0}\right)=-\alpha\left(c_{0}\right)^{-g_{0}\left(c_{0}\right)} \text {. }
$$

Example 1.4.3. We here give some easy examples of stable quadratic modules.

(1) A stable quadratic module with trivial bracket $\langle\cdot, \cdot\rangle$ is the same as an abelian group homomorphism $f$ :

$$
B \otimes B \stackrel{0}{\longrightarrow} A \stackrel{f}{\longrightarrow} B .
$$


(2) If $G$ is a group of nilpotency class 2 and $H \subset G$ is a subgroup containing commutators, $[G, G] \subset H$, then

$$
G^{a b} \otimes G^{a b} \stackrel{\langle\cdot, \cdot\rangle}{\longrightarrow} H \longleftrightarrow G, \quad\langle x, y\rangle=[y, x],
$$

is a stable quadratic module.

(3) The following stable quadratic module consists of abelian groups but has a non-trivial bracket

$$
\mathbb{Z} \otimes \mathbb{Z} \stackrel{\langle\cdot, \cdot\rangle}{\longrightarrow} \mathbb{Z} / 2 \stackrel{0}{\longrightarrow} \mathbb{Z}, \quad\langle 1,1\rangle=1 .
$$

(4) More generally, if $R$ is a commutative ring and $R^{\times}$is the (multiplicative) group of units we can consider the stable quadratic module

$$
\mathbb{Z} \otimes \mathbb{Z} \stackrel{\langle\cdot, \cdot\rangle}{\longrightarrow} R^{\times} \stackrel{0}{\longrightarrow} \mathbb{Z}, \quad\langle 1,1\rangle=-1 .
$$

Stable quadratic modules are closely related to Picard groupoids.

Definition 1.4.4. The Picard groupoid $\Gamma C_{*}$ associated to a stable quadratic module $C_{*}$ is defined as follows. The set of objects is $C_{0}$. The set of all morphisms is the semidirect product $C_{0} \ltimes C_{1}$, i.e. the cartesian product with the following group structure:

$$
\left(c_{0}, c_{1}\right)+\left(c_{0}^{\prime}, c_{1}^{\prime}\right)=\left(c_{0}+c_{0}^{\prime}, c_{1}^{c_{0}^{\prime}}+c_{1}^{\prime}\right) .
$$

The source and target of $\left(c_{0}, c_{1}\right) \in C_{0} \ltimes C_{1}$ are

$$
\left(c_{0}, c_{1}\right): c_{0}+\partial\left(c_{1}\right) \longrightarrow c_{0} .
$$

Composition of morphisms is defined as

$$
\left(c_{0}, c_{1}\right) \circ\left(c_{0}+\partial\left(c_{1}\right), c_{1}^{\prime}\right)=\left(c_{0}, c_{1}+c_{1}^{\prime}\right) .
$$

The tensor product is simply the sum + on both objects and morphisms, and the tensor unit is $I=0$. It is strictly associative and unital. Symmetry constraints are defined by the bracket

$$
\left(c_{0}^{\prime}+c_{0},\left\langle c_{0}^{\prime}, c_{0}\right\rangle\right): c_{0}+c_{0}^{\prime} \longrightarrow c_{0}^{\prime}+c_{0} .
$$

Notice that identity morphisms are given by

$$
1_{c_{0}}=\left(c_{0}, 0\right) .
$$

A morphism $f: C_{*} \rightarrow D_{*}$ induces a strict tensor functor $\Gamma f: \Gamma C_{*} \rightarrow$ $\Gamma D_{*}$ given on objects by $f_{0}$ and on morphisms by $f_{0} \ltimes f_{1}$. A homotopy $\alpha: f \Rightarrow g$ between two morphisms $f, g: C_{*} \rightarrow D_{*}$ induces a tensor natural transformation $\Gamma \alpha: \Gamma f \Rightarrow \Gamma g$ defined by $(\Gamma \alpha)\left(c_{0}\right)=\left(g_{0}\left(c_{0}\right), \alpha\left(c_{0}\right)\right)$. In this way, $\Gamma$ defines a 2 -functor from the 2-category of stable quadratic modules to the 2-category of Picard groupoids. 
Definition 1.4.5. The homotopy groups of a stable quadratic module $C_{*}$ are

$$
\pi_{0}\left(C_{*}\right)=C_{0} / \partial\left(C_{1}\right), \quad \pi_{1}\left(C_{*}\right)=\operatorname{Ker} \partial
$$

The $k$-invariant is the natural homomorphism

$$
\begin{aligned}
\eta: \pi_{0}\left(C_{*}\right) \otimes \mathbb{Z} / 2 \longrightarrow \pi_{1}\left(C_{*}\right), \\
{\left[c_{0}\right] \otimes 1 \longmapsto\left\langle c_{0}, c_{0}\right\rangle . }
\end{aligned}
$$

Homotopy groups are functors from the category squad of stable quadratic modules, and the $k$-invariant is a natural transformation. A weak equivalence or quasi-isomorphism is a morphism which induces isomorphisms on homotopy groups. The homotopy category Hosquad is obtained from squad by formally inverting quasi-isomorphisms. Two stable quadratic modules with isomorphic $\pi_{0}$ and $\pi_{1}$ are weakly equivalent if and only if they have isomorphic $k$-invariant, cf. [Sin, Chapitre 1 $\S 1$, Proposition 10].

A stable quadratic module $C_{*}$ is 0 -free if $C_{0}=\langle E\rangle^{\text {nil }}$ is the nilpotent group of class two freely generated by a set $E$, i.e. the quotient of the free non-abelian group $\langle E\rangle$ by triple commutators. We denote by $\mathbf{s q u a d}_{0}$ the full subcategory spanned by 0 -free objects

Remark 1.4.6. Notice that $\pi_{0}\left(C_{*}\right)$ is the group of isomorphism classes of objects in $\Gamma C_{*}$, and $\pi_{1}\left(C_{*}\right)$ is the automorphism group of the tensor unit in $\Gamma C_{*}$. The $k$-invariant measures the deviation of $\Gamma C_{*}$ from being strictly commutative. The homotopy category Ho squad is equivalent to the category $\operatorname{squad}_{0} / \simeq$ obtained by dividing out homotopies from squad $_{0}$.

Example 1.4.7. The homotopy groups and the $k$-invariants of the stable quadratic modules in Example 1.4.3 are:

(1) $\pi_{0}=\operatorname{Coker} f, \pi_{1}=\operatorname{Ker} f$, and the $k$-invariant vanishes.

(2) $\pi_{0}=G / H, \pi_{1}=0$, and the $k$-invariant vanishes for obvious reasons.

(3) $\pi_{0}=\mathbb{Z}, \pi_{1}=\mathbb{Z} / 2$, and the $k$-invariant is the natural projection $\mathbb{Z} \rightarrow \mathbb{Z} / 2$.

(4) $\pi_{0}=\mathbb{Z}, \pi_{1}=R^{\times}$, and the $k$-invariant is $\mathbb{Z} \rightarrow R^{\times}: 1 \mapsto-1$.

The following lemma about homotopies is very useful to deform morphisms, see [Wit1, Lemmas 2.1.13 and 2.1.14]. 
Lemma 1.4.8. Let $g: C_{*} \rightarrow D_{*}$ be a morphism of stable quadratic modules with $C_{0}=\langle E\rangle^{\text {nil }}$. Any map $E \rightarrow D_{1}$ extends to a map

$$
\alpha: C_{0} \longrightarrow D_{1}
$$

satisfying

$$
\alpha\left(c_{0}+c_{0}^{\prime}\right)=\alpha\left(c_{0}\right)^{g_{0}\left(c_{0}^{\prime}\right)}+\alpha\left(c_{0}^{\prime}\right), \quad c_{0}, c_{0}^{\prime} \in C_{0} .
$$

Moreover, there is a unique morphism $f=g+\alpha: C_{*} \rightarrow D_{*}$, defined as

$$
f\left(c_{0}\right)=g\left(c_{0}\right)+\partial \alpha\left(c_{0}\right), \quad f\left(c_{1}\right)=g\left(c_{1}\right)+\alpha \partial\left(c_{1}\right), \quad c_{i} \in C_{i}, i=0,1,
$$

such that $\alpha$ is a homotopy $\alpha: f \Rightarrow g$.

Definition 1.4.9. A strong deformation retraction is a special kind of homotopy equivalence between stable quadratic modules, given by a diagram

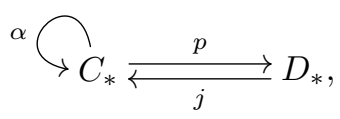

where $p$ and $j$ are morphisms such that $p j=1_{D_{*}}$ and $\alpha: j p \Rightarrow 1_{C_{*}}$ is a homotopy satisfying $\alpha j=0$ and $p \alpha=0$.

The following lemma will help us to define strong deformation retractions.

Lemma 1.4.10. Consider a 0 -free stable quadratic module $C_{*}$ with $C_{0}=$ $\langle E\rangle^{\text {nil }}$. Moreover, suppose $j: D_{*} \rightarrow C_{*}$ is a levelwise injective morphism of stable quadratic modules such that $D_{0}=\left\langle E^{\prime}\right\rangle^{\text {nil }}, E^{\prime} \subset E$, and $j_{0}$ is induced by the inclusion. Let $\alpha: E \rightarrow C_{1}$ be a map. Assume that the morphism $f=1_{C_{*}}+\alpha: C_{*} \rightarrow C_{*}$ from Lemma 1.4 .8 factors as $f=j p$, and that the homotopy $\alpha$ satisfies

$$
\alpha\left(e^{\prime}\right)=0, \quad e^{\prime} \in E^{\prime}, \quad \alpha(e+\partial \alpha(e))=0, \quad e \in E .
$$

Then $\alpha, p$, and $j$ define a strong deformation retraction.

Proof: The only non-obvious formulas are $p \alpha=0$ and $p j=1_{D_{*}}$. Since $j$ is levelwise injective, it is enough to check that $f \alpha=0$ and $f j=j$. These hold since, by definition of $f=1_{C_{*}}+\alpha$, we have $f \alpha(e)=\alpha(e)+\alpha \partial \alpha(e)=$ $\alpha(e+\partial \alpha(e))=0$ and $f\left(e^{\prime}\right)=e^{\prime}+\partial \alpha\left(e^{\prime}\right)=e^{\prime}$.

We now consider the left adjoint of the functor sending a stable quadratic module $C_{*}$ to the pair of sets $\left(C_{0}, C_{1}\right)$, see [MT1, Appendix A]. Stable quadratic modules in the image of this left adjoint are said to be free. 
Definition 1.4.11. The free stable quadratic module $F_{*}^{s}\left(E_{0}, E_{1}\right)$ on a pair of sets $\left(E_{0}, E_{1}\right)$ can be constructed as follows: $F_{0}^{s}\left(E_{0}, E_{1}\right)=$ $\left\langle E_{0} \sqcup E_{1}\right\rangle^{\text {nil }}$. Moreover, if we denote $\langle E\rangle^{a b}$ the free abelian group on a set $E$, then

$$
F_{1}^{s}\left(E_{0}, E_{1}\right)=\left\langle E_{0}\right\rangle^{a b} \otimes \mathbb{Z} / 2 \times \wedge^{2}\left\langle E_{0}\right\rangle^{a b} \times\left\langle E_{0}\right\rangle^{a b} \otimes\left\langle E_{1}\right\rangle^{a b} \times\left\langle E_{1}\right\rangle^{\mathrm{nil}} .
$$

The homomorphism $\partial$ and the bracket $\langle\cdot, \cdot\rangle$ in $F_{*}^{s}\left(E_{0}, E_{1}\right)$ are defined by the following formulas:

$$
\begin{aligned}
\partial\left(e_{0} \otimes 1, e_{0}^{\prime} \wedge e_{0}^{\prime \prime}, e_{0}^{\prime \prime \prime} \otimes e_{1}, e_{1}^{\prime}\right) & =\left[e_{0}^{\prime \prime}, e_{0}^{\prime}\right]+\left[e_{1}, e_{0}^{\prime \prime \prime}\right]+e_{1}^{\prime} ; \\
\left\langle e_{0}, e_{0}\right\rangle & =\left(e_{0} \otimes 1,0,0,0\right) ; \\
\text { if } e_{0} \neq e_{0}^{\prime} \text { then } \quad\left\langle e_{0}, e_{0}^{\prime}\right\rangle & =\left(0, e_{0} \wedge e_{0}^{\prime}, 0,0\right) ; \\
\left\langle e_{0}, e_{1}\right\rangle & =\left(0,0, e_{0} \otimes e_{1}, 0\right) ; \\
\left\langle e_{1}, e_{1}^{\prime}\right\rangle & =\left(0,0,0,\left[e_{1}^{\prime}, e_{1}\right]\right) .
\end{aligned}
$$

Given two sets of relations $R_{i} \subset F_{i}^{s}\left(E_{0}, E_{1}\right), i=0,1$, the stable quadratic module $C_{*}$ with generators $\left(E_{0}, E_{1}\right)$ and relations $\left(R_{0}, R_{1}\right)$ is defined as follows: $C_{0}$ is the quotient of $F_{0}^{s}\left(E_{0}, E_{1}\right)$ by the normal subgroup $N_{0}$ generated by $R_{0} \cup \partial R_{1}$, and $C_{1}$ is the quotient of $F_{1}^{s}\left(E_{0}, E_{1}\right)$ by the normal subgroup generated by $R_{1}$ and $\left\langle F_{0}^{s}\left(E_{0}, E_{1}\right), N_{0}\right\rangle$. The homomorphism $\partial$ and the bracket $\langle\cdot, \cdot\rangle$ on $F_{*}^{s}\left(E_{0}, E_{1}\right)$ induce a structure of a stable quadratic module on $C_{*}$. This is the unique structure for which the natural projection $F_{*}^{s}\left(E_{0}, E_{1}\right) \rightarrow C_{*}$ is a morphism of stable quadratic modules.

Stable quadratic modules defined by a presentation satisfy the obvious universal property.

1.5. Universal determinant functors. In this section we present universal determinant functors for all cases considered above. We will actually construct them by using presentations of stable quadratic modules.

Definition 1.5.1. Let $\mathscr{W}$ be a Waldhausen category. We define the stable quadratic module $\mathcal{D}_{*}(\mathscr{W})$ by generators

(G1) $[X]$ for any object, in dimension 0 ,

(G2) $\left[X \stackrel{\sim}{\rightarrow} X^{\prime}\right]$ for any weak equivalence, in dimension 1 ,

(G3) $[\Delta]$ for any cofiber sequence as in (1.2.2), in dimension 1, and relations

(R1) $\partial\left[X \stackrel{\sim}{\rightarrow} X^{\prime}\right]=-\left[X^{\prime}\right]+[X]$,

(R2) $\partial[\Delta]=-[Y]+\left[C^{f}\right]+[X]$,

(R3) $[0]=0$ for the zero object, 
(R4) $[X \stackrel{1}{\rightarrow} X]=0$ for any object,

(R5) $[X \stackrel{1}{\longmapsto} X \rightarrow 0]=0=[0 \longmapsto X \stackrel{1}{\rightarrow} X]$ for any object,

(R6) for any pair of composable weak equivalences $X \stackrel{\sim}{\rightarrow} Y \stackrel{\sim}{\rightarrow} Z$,

$$
[X \stackrel{\sim}{\longrightarrow} Z]=[Y \stackrel{\sim}{\longrightarrow} Z]+[X \stackrel{\sim}{\longrightarrow} Y]
$$

(R7) for any weak equivalence of cofiber sequences $\Phi: \Delta \stackrel{\sim}{\rightarrow} \Delta^{\prime}$ as in (1.2.4),

$$
\left[X \stackrel{\sim}{\longrightarrow} X^{\prime}\right]+\left[C^{f \stackrel{\sim}{\longrightarrow}} C^{f^{\prime}}\right]^{[X]}=-\left[\Delta^{\prime}\right]+\left[Y \stackrel{\sim}{\longrightarrow} Y^{\prime}\right]+[\Delta],
$$

(R8) for any staircase diagram $\Theta$ as in (1.2.5),

$$
\left[\Delta_{g}\right]+\left[\Delta_{f}\right]=\left[\Delta_{g f}\right]+[\widetilde{\Delta}]^{[X]}
$$

(R9) for any two objects $X$ and $Y$,

$$
\langle[X],[Y]\rangle=-\left[\Delta_{2}\right]+\left[\Delta_{1}\right],
$$

where $\Delta_{1}$ and $\Delta_{2}$ are the cofiber sequences in (1.2.6).

This stable quadratic module was first considered in [MT1].

The stable quadratic module $\mathcal{D}_{*}^{\text {der }}(\mathscr{W})$ is defined by almost the same presentation, modified in the following way. We have generators (G2) for all isomorphisms $X \cong X^{\prime}$ in Ho $\mathscr{W},(\mathrm{R} 1)-(\mathrm{R} 5),(\mathrm{R} 8)$, and (R9) remain the same, (R6) must hold for any pair of composable isomorphisms in Ho $\mathscr{W}$, and (R7) is required for all cofiber sequence isomorphisms $\Phi: \Delta \cong \Delta^{\prime}$ in $\operatorname{Ho}\left(S_{2} \mathscr{W}\right)$, see [Mur, Definition 5.4].

Given a triangulated category $\mathscr{T}$ we define the stable quadratic module $\mathcal{D}_{*}\left({ }^{b} \mathscr{T}\right)$ as follows. The definition is by generators and relations as above. Generators (G1)-(G3) correspond to objects, isomorphisms $X \cong X^{\prime}$, and distinguished triangles $\Delta$ as in (1.3.1), respectively. Relations (R1)-(R4) are the same, (R5) is

(R5) $[X \stackrel{1}{\rightarrow} X \rightarrow 0 \rightarrow \Sigma X]=0=[0 \rightarrow X \stackrel{1}{\rightarrow} X \rightarrow 0]$ for any object, relation (R6) is imposed for any pair of composable isomorphisms, (R7) is required for any exact triangle isomorphism $\Phi: \Delta \cong \Delta^{\prime}$ as in (1.3.6), (R8) must hold for any octahedron $\Theta$ as in (1.3.2), and the exact triangles $\Delta_{1}$ and $\Delta_{2}$ in (R9) are defined in (1.3.7).

The stable quadratic module $\mathcal{D}_{*}\left({ }^{d} \mathscr{T}\right)$ is presented as $\mathcal{D}_{*}\left({ }^{b} \mathscr{T}\right)$, except that we only require (R8) for special octahedra.

For the presentation of $\mathcal{D}_{*}\left({ }^{v} \mathscr{T}\right)$, we allow all virtual triangles as generators (G3), (R7) must hold for any virtual triangle isomorphism, and (R8) for any virtual octahedron $\Theta$.

If $\mathscr{T}_{3}$ is a 3 -pretriangulated category, the stable quadratic module $\mathcal{D}_{*}\left({ }^{s} \mathscr{T}_{3}\right)$ is again presented as $\mathcal{D}_{*}\left({ }^{b} \mathscr{T}\right)$, but we only impose (R8) for distinguished octahedra. 
Remark 1.5.2. All these stable quadratic modules are 0-free. The degree 0 group is free of nilpotence class 2 with basis given by the set of non-trivial objects.

The presentations above are not minimal. Relation (R3) follows from (R2) and (R5), (R4) follows from (R6), and (R5) is equivalent to

$\left(\mathrm{R} 5^{\prime}\right)[0 \longmapsto 0 \rightarrow 0]=0$ in $\mathcal{D}_{*}(\mathscr{W})$.

$\left(\mathrm{R}^{\prime}\right)[0 \rightarrow 0 \rightarrow 0 \rightarrow \Sigma 0]=0$ in $\mathcal{D}_{*}(\bullet \mathscr{T}), \bullet=b, d, s, v$.

In all cases, if (R9) holds for a given coproduct of two objects then it also holds for any other coproduct. This follows from the uniqueness of coproducts up to isomorphism and the rest of the relations.

For $\mathcal{D}_{*}(\mathscr{W})$ and $\mathcal{D}_{*}^{\text {der }}(\mathscr{W})$ it is enough to impose $(\mathrm{R} 8)$ for only one staircase completion of each two composable cofibrations $X \longmapsto Y \longmapsto Z$, as any two completions are isomorphic. Similarly, for $\mathcal{D}_{*}\left({ }^{s} \mathscr{T}_{3}\right)$ is enough to impose (R8) for only one distinguished octahedron completing each pair of composable morphisms $X \rightarrow Y \rightarrow Z$. This is not the case for ordinary triangulated categories, see [Kün].

The following proposition provides smaller presentations in some cases. It follows from Proposition 4.6.11.

Proposition 1.5.3. Let $\mathscr{W}$ be a Waldhausen category where weak equivalences are isomorphisms and $\mathscr{T}$ a (strongly) triangulated category. Then $\mathcal{D}_{*}(\mathscr{W})$ and $\mathcal{D}_{*}(\bullet \mathscr{T})$, $=b, d, s, v$, have a presentation with generators $(\mathrm{G} 1)$ and $(\mathrm{G} 3)$ and relations $(\mathrm{R} 2),\left(\mathrm{R} 5^{\prime}\right),(\mathrm{R} 8)$, and $(\mathrm{R} 9)$.

The following theorem is the main result of this paper.

Theorem 1.5.4. Let $\mathscr{W}$ be a Waldhausen category, $\mathscr{T}$ a triangulated category, and $\mathscr{T}_{3}$ a 3-pretriangulated category. We have:

- a determinant functor det: $\mathscr{W} \rightarrow \Gamma \mathcal{D}_{*}(\mathscr{W})$,

- a derived determinant functor det: $\mathscr{W} \rightarrow \Gamma \mathcal{D}_{*}^{\text {der }}(\mathscr{W})$,

- a Breuning determinant functor det: $\mathscr{T} \rightarrow \Gamma \mathcal{D}_{*}\left({ }^{b} \mathscr{T}\right)$,

- a special determinant functor det: $\mathscr{T} \rightarrow \Gamma \mathcal{D}_{*}\left({ }^{d} \mathscr{T}\right)$,

- a virtual determinant functor det: $\mathscr{T} \rightarrow \Gamma \mathcal{D}_{*}(v \mathscr{T})$,

- a determinant functor det: $\mathscr{T}_{3} \rightarrow \Gamma \mathcal{D}_{*}\left({ }^{s} \mathscr{T}_{3}\right)$,

all of which are universal. They are defined (in the notation of Definition 1.4.4) by: 
- $\operatorname{det}(X)=[X]$ for any object,

- $\operatorname{det}\left(X \rightarrow X^{\prime}\right)=\left(\left[X^{\prime}\right],\left[X \rightarrow X^{\prime}\right]\right)$ for any weak equivalence or isomorphism,

- $\operatorname{det}(\Delta)=([Y],[\Delta])$ for any cofiber sequence, distinguished triangle or virtual triangle, as in (1.2.2) or (1.3.1).

Moreover, if we simply regard the targets as categorical groups, forgetting the symmetry, these determinant functors are also universal among noncommutative determinant functors.

This result follows from Theorem 4.3.4 and Corollary 4.4.5.

If our Waldhausen or (strongly) triangulated category has functorial coproducts, we may use a quotient of the stable quadratic module in Definition 1.5.1 instead.

Definition 1.5.5. A category has functorial coproducts if it has a monoidal structure + which is strictly associative and unital, the unit 0 is an initial object, and the following diagram is a coproduct for any two objects $X$ and $Y$,

$$
X=X+0 \longrightarrow X+Y \longleftarrow 0+Y=Y .
$$

If $\mathscr{W}$ and $\mathscr{T}$ are a Waldhausen category and a (strongly) triangulated category with functorial coproducts, respectively, we define the stable quadratic modules $\mathcal{D}_{*}^{+}(\mathscr{W})$ and $\mathcal{D}_{*}^{+}(\bullet \mathscr{T}), \bullet=b, d, v, s$, as the quotient of $\mathcal{D}_{*}(\mathscr{W})$ and $\mathcal{D}_{*}(\bullet \mathscr{T})$ by the following extra relation,

(R10) $[Y \longmapsto X+Y \rightarrow X]=0$ for any pair of objects $X$ and $Y$ in $\mathscr{W}$.

(R10) $[Y \rightarrow X+Y \rightarrow X \stackrel{0}{\rightarrow} \Sigma Y]=0$ for any pair of objects $X$ and $Y$ in $\mathscr{T}$.

Remark 1.5.6. In $\mathcal{D}_{*}^{+}(\mathscr{W})$ and $\mathcal{D}_{*}^{+}(\mathscr{T})$, for any two objects $X$ and $Y$, we have

$$
\begin{aligned}
{[X+Y] } & =[X]+[Y], \\
\langle[X],[Y]\rangle & =[Y+X \cong X+Y] .
\end{aligned}
$$

Actually, (R7), (R9'), and (R10) imply (R9), compare [MT2, Remark 4.1]. Notice also that $\left(\mathrm{R} 5^{\prime}\right)$ is a special case of (R10).

In $\mathcal{D}_{*}^{+}(\mathscr{T})$, given two distinguished (or virtual if $\bullet=v$ ) triangles,

$$
\begin{aligned}
{\left[X+X^{\prime} \stackrel{f+f^{\prime}}{\longrightarrow} Y+Y^{\prime} \stackrel{i+i^{\prime}}{\longrightarrow} Z+Z^{\prime} \stackrel{q+q^{\prime}}{\longrightarrow} \Sigma X+\Sigma X^{\prime}\right] } \\
=[X \stackrel{f}{\longrightarrow} Y \stackrel{i}{\longrightarrow} Z \stackrel{q}{\longrightarrow} \Sigma X]^{\left[Y^{\prime}\right]} \\
\quad+\left[X^{\prime} \stackrel{f^{\prime}}{\longrightarrow} Y^{\prime} \stackrel{i^{\prime}}{\longrightarrow} Z^{\prime} \stackrel{q^{\prime}}{\longrightarrow} \Sigma X^{\prime}\right]+\left\langle[X],\left[Z^{\prime}\right]\right\rangle .
\end{aligned}
$$


See Corollary 4.6.16 below. See also [MT2, Lemma 4.8] for the corresponding result in $\mathcal{D}_{*}^{+}(\mathscr{W})$.

Proposition 1.5.7. Let $\mathscr{W}$ and $\mathscr{T}$ be a Waldhausen category and a (strongly) triangulated category with functorial coproducts, respectively. Assume the set of objects is free as a monoid under + in both cases. Then the natural projection,

$$
\mathcal{D}_{*}(\mathscr{W}) \longrightarrow \mathcal{D}_{*}^{+}(\mathscr{W}) ; \quad \mathcal{D}_{*}(\bullet \mathscr{T}) \longrightarrow \mathcal{D}_{*}^{+}(\bullet \mathscr{T}), \quad \bullet=b, d, v, s ;
$$

is a weak equivalence. It is actually part of a strong deformation retraction.

This follows from Proposition 4.6.13 below.

Remark 1.5.8. Under the hypotheses of the previous proposition, $\mathcal{D}_{*}^{+}(\mathscr{W})$ and $\mathcal{D}_{*}^{+}(\mathscr{T})$ are also 0 -free. The degree 0 group of nilpotency class 2 is freely generated by any basis of the free monoid of objects.

Let us consider a further simplification for additive categories. It will lead to some explicit computations.

Proposition 1.5.9. Let $\mathscr{A}$ be an additive category, regarded as a split exact category. Suppose it satisfies the hypotheses of Proposition 1.5.\%. Then $\mathcal{D}_{*}^{+}(\mathscr{A})$ has a presentation with generators (G1) and (G2) and relations (R1), (R6), (R9'), and

$\left(\mathrm{R} 7^{\prime}\right)\left[f: X \cong X^{\prime}\right]^{\left[Y^{\prime}\right]}+\left[g: Y \cong Y^{\prime}\right]=\left[f+g: X+Y \cong X^{\prime}+Y^{\prime}\right]$.

Proof: Applying (R7) and (R10) to

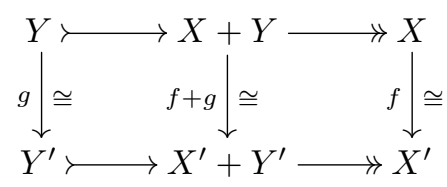

we obtain $\left(\mathrm{R} 7^{\prime}\right)$.

Given a short exact sequence $\Delta: X \stackrel{f}{\longmapsto} Y \stackrel{p}{\rightarrow} C$, a splitting

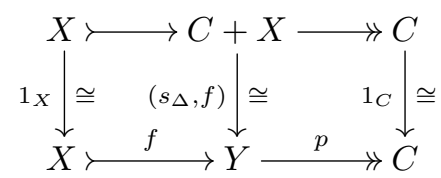

and (R7) yield

$$
[\Delta]=\left[\left(s_{\Delta}, f\right)\right] .
$$


Such a splitting is not unique, but if $\left(s_{\Delta}^{\prime}, f\right)$ is another then there is a unique $h: C \rightarrow X$ such that $s_{\Delta}^{\prime}=s_{\Delta}+f h$, i.e.

$$
\left(s_{\Delta}^{\prime}, f\right)=\left(s_{\Delta}, f\right)\left(\begin{array}{cc}
1_{C} & 0 \\
h & 1_{X}
\end{array}\right) \text {. }
$$

Hence, by (R6),

$$
\left[\left(s_{\Delta}^{\prime}, f\right)\right]=\left[\left(s_{\Delta}, f\right)\right]+\left[\left(\begin{array}{cc}
1_{C} & 0 \\
h & 1_{X}
\end{array}\right)\right]=\left[\left(s_{\Delta}, f\right)\right],
$$

compare the proof of [Ran, Proposition 1.1] for the vanishing of $\left[\left(\begin{array}{cc}1_{C} & 0 \\ h & 1_{X}\end{array}\right)\right]$.

Let us check that $(\mathrm{R} 7)$ and $(\mathrm{R} 8)$ follow from the relations in the statement. Given

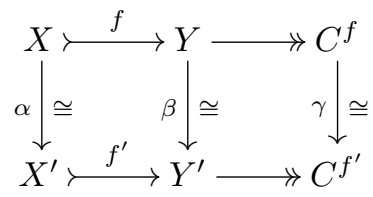

we can choose compatible splittings defined by $s_{\Delta}$ and $s_{\Delta^{\prime}}=\beta s_{\Delta} \gamma^{-1}$. Hence by (R6) and $\left(\mathrm{R} 7^{\prime}\right)$

$$
\begin{aligned}
{\left[\left(s_{\Delta^{\prime}}, f^{\prime}\right)\right] } & =\left[\left(\beta s_{\Delta} \gamma^{-1}, \beta f \alpha^{-1}\right)\right]=\left[\beta\left(s_{\Delta}, f\right)\left(\gamma^{-1}+\alpha^{-1}\right)\right] \\
& =[\beta]+\left[\left(s_{\Delta}, f\right)\right]+\left[\gamma^{-1}\right]^{[X]}+\left[\alpha^{-1}\right] \\
& =[\beta]+\left[\left(s_{\Delta}, f\right)\right]-[\gamma]^{[X]}-[\alpha] .
\end{aligned}
$$

This is (R7).

Given a staircase diagram

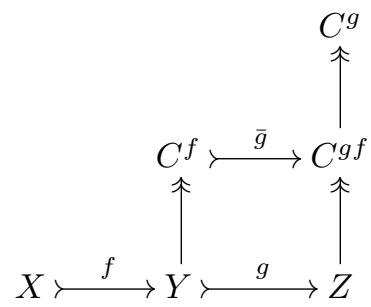

we can choose splittings defined by morphisms $s_{\Delta_{f}}, s_{\Delta_{g}}, s_{\Delta_{g f}}$, and $s_{\widetilde{\Delta}}$, such that $s_{\Delta_{g}}=s_{\Delta_{g f}} s_{\widetilde{\Delta}}$ and $s_{\Delta_{g f}} \bar{g}=g s_{\Delta_{f}}$. Therefore,

$$
\left(s_{\Delta_{g}}, g\right)\left(1_{C^{g}}+\left(s_{\Delta_{f}}, f\right)\right)=\left(s_{\Delta_{g f}}, g f\right)\left(\left(s_{\widetilde{\Delta}}, \bar{g}\right)+1_{X}\right) .
$$

Now (R8) follows from (R6) and $\left(\mathrm{R} 7^{\prime}\right)$ applied to this formula.

An additive category $\mathscr{A}$ satisfies the Krull-Remak-Schmidt theorem if any object in $\mathscr{A}$ is a direct sum of indecomposables and, up to a permutation, the indecomposable components in such a direct sum are uniquely determined up to isomorphism. 
Corollary 1.5.10. Let $\mathscr{A}$ be an additive category satisfying the KrullRemak-Schmidt theorem. Denote $S$ a skeletal set of indecomposables. Then $\mathcal{D}_{*}(\mathscr{A})$ is weakly equivalent to

$$
K_{0}(\mathscr{A}) \otimes K_{0}(\mathscr{A}) \stackrel{\langle\cdot \cdot \cdot\rangle}{\longrightarrow} K_{1}(\mathscr{A}) \stackrel{0}{\longrightarrow} K_{0}(\mathscr{A})=\langle S\rangle^{a b},
$$

where $\langle[X],[X]\rangle=\left[-1_{X}\right]$ and $\langle[X],[Y]\rangle=0$ for $X, Y \in S, X \neq Y$.

Proof: We can suppose without loss of generality that $\mathscr{A}$ has functorial coproducts and that the monoid of objects is freely generated by $S$, see [MT2, Proposition 4.3]. By Remark 1.5.8, $\mathcal{D}_{0}^{+}(\mathscr{A})=\langle S\rangle^{\text {nil }}$. Moreover, by the Krull-Remak-Schmidt theorem the image of $\partial$ is the commutator subgroup and $\pi_{0} \mathcal{D}_{*}^{+}(\mathscr{A})=\langle S\rangle^{a b}=K_{0}(\mathscr{A})$. The commutator bracket induces a monomorphism $\wedge^{2}\langle S\rangle^{a b} \hookrightarrow\langle S\rangle^{\text {nil }},[X] \wedge[Y] \mapsto[[X],[Y]]$. This injection factors through $\mathcal{D}_{1}^{+}(\mathscr{A})$. In order to define a factorization, we need to choose a total order $\leq$ in $S$. A factorization is given by

$$
\wedge^{2}\langle S\rangle^{a b} \longleftrightarrow \mathcal{D}_{1}^{+}(\mathscr{A}), \quad[X] \wedge[Y] \longmapsto\langle[Y],[X]\rangle, \quad X<Y \in S .
$$

Dividing out $\wedge^{2}\langle S\rangle^{a b}$ from $\mathcal{D}_{*}^{+}(\mathscr{A})$ we obtain a weakly equivalent stable quadratic module $C_{*}$ with $C_{0}=\pi_{0} C_{0}=\langle S\rangle^{a b}=K_{0}(\mathscr{A})$ and $\partial=0$. We must now identify $C_{1}$ and the bracket. Proposition 1.5 .9 and the explicit description of a stable quadratic module defined by a presentation show that $C_{1}$ is the abelian group defined by generators $[f: X \cong Y]$ for each isomorphism in $\mathscr{A}$, and relations

- $2 \cdot\left[\left(\begin{array}{cc}0 & 1_{X} \\ 1_{X} & 0\end{array}\right)\right]=0$ for $X \in S$,

- $\left[\left(\begin{array}{cc}0 & 1_{X} \\ 1_{Y} & 0\end{array}\right)\right]=0$ for $X, Y \in S, X \neq Y$,

- $[g: Y \cong Z]+[f: X \cong Y]=[g f]$ for any two composable isomorphisms,

- $\left[f: X \cong X^{\prime}\right]+\left[g: Y \cong Y^{\prime}\right]=[f+g]$ for any two isomorphisms.

The first relation follows from the third one. Hence, $C_{1}$ is the quotient of Ranicki's $K_{1}^{\text {iso }}(\mathscr{A})$ by the isomorphisms of the canonical structure in the sense of [Ran, §5] defined by the permutations of different $S$ factors in a direct sum decomposition. Therefore, $C_{1}=K_{1}(\mathscr{A})$ by [Ran, Proposition 5.3]. The computation of the non-trivial brackets follows as in [MT1, Corollary 1.10].

Remark 1.5.11. This corollary can be applied to several additive categories: modules of finite length over a ring, finitely generated projective modules over a semiperfect ring, finitely generated free modules over a ring $R$ with the invariant basis number property, etc. In particular, if $R$ 
is a commutative local ring or an Euclidean domain we obtain the stable quadratic module in Example 1.4.3(4).

In this section, we have obtained a connection between determinant functors and $K$-theory through an explicit computation of a generic but simple example. In the following section we will see that this relation is much broader.

1.6. The connection to $\boldsymbol{K}$-theory. Let Ho $\mathbf{S p e c}_{0}$ be the full coreflective subcategory of the stable homotopy category spanned by connective spectra, i.e. spectra with trivial homotopy groups in negative dimensions.

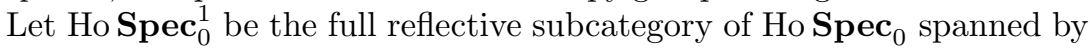
spectra with homotopy groups concentrated in dimensions 0 and 1 . The reflection functor $\mathrm{Ho} \mathbf{S p e c}_{0} \rightarrow \mathrm{Ho}_{\mathbf{S p e c}}^{1}$ takes a connective spectrum to its 1-type. It is well known (see for example [Boy]) that the homotopy category of Picard groupoids is equivalent to $\mathrm{Ho} \mathbf{S p e c}_{0}^{1}$, and the equivalence is compatible with the corresponding notions of homotopy groups and $k$-invariant. Recall that, on homotopy groups of spectra, the $k$-invariant is simply the action of the stable Hopf map. There are several ways of realizing this equivalence. The equivalence in [MT1] between Ho Spec $_{0}^{1}$ and the homotopy category of stable quadratic modules Ho squad is particularly well adapted to the goal of this paper.

Lemma 1.6.1 ([MT1, Lemma 4.22]). There is a functor

$$
\lambda_{0}: \mathrm{Ho}_{\mathrm{Spec}} \longrightarrow \text { Ho squad }
$$

together with natural isomorphisms

$$
\pi_{i} \lambda_{0} X \cong \pi_{i} X, \quad i=0,1,
$$

compatible with the $k$-invariants, which restricts to an equivalence of categories

$$
\lambda_{0}: \operatorname{HoSpec}_{0}^{1} \stackrel{\sim}{\longrightarrow} \text { Ho squad. }
$$

Therefore the functor $\lambda_{0}$ can be regarded as an algebraic model for the 1-type of a connective spectrum.

Example 1.6.2. If $S$ is the sphere spectrum, its first two homotopy groups and the $k$-invariant are as in Example 1.4.7(3), hence $\lambda_{0} S$ is weakly equivalent to Example 1.4.3(3).

If $R$ is a commutative local ring, the first two homotopy groups of its connective $K$-theory spectrum $K(R)$ are as in Example 1.4.7(4). The $k$-invariant connecting them is also as described therein. This can be easily checked by looking at the canonical map of spectra $S \rightarrow K(R)$, 
the unit of the ring spectrum structure on $K(R)$, which is the identity in $\pi_{0}$ and $\mathbb{Z} / 2 \rightarrow R^{\times}: 1 \mapsto-1$ in $\pi_{1}$. Therefore $\lambda_{0} K(R)$ is weakly equivalent to Example 1.4.3(4).

Examples of connective spectra are Quillen's $K$-theory of an exact category $K(\mathscr{E})$ [Qui] (although there is also a non-connective version), Waldhausen's $K$-theory of a category with cofibrations and weak equivalences $K(\mathscr{W})$ [Wal2], Garkusha's derived $K$-theory of an exact category $D K(\mathscr{E})$ [Gar1], its generalization for a Waldhausen category $D K(\mathscr{W})$ [Mur], Maltsiniotis's $K$-theory of a strongly triangulated category $K\left({ }^{s} \mathscr{T}_{\infty}\right)[\mathbf{M a l 1}]$, and two of Neeman's $K$-theories of a triangulated category, $K\left({ }^{d} \mathscr{T}\right)$ and $K\left({ }^{v} \mathscr{T}\right)$ [Nee2], see Subsection 4.5 below.

Theorem 1.6.3. Let $\mathscr{W}$ be a Waldhausen category, $\mathscr{T}$ a triangulated category, and $\mathscr{T}_{\infty}$ a strongly triangulated category. There are natural isomorphisms in Ho squad:

$$
\begin{array}{ll}
\mathcal{D}_{*}(\mathscr{W}) \cong \lambda_{0} K(\mathscr{W}), & \mathcal{D}_{*}^{\text {der }}(\mathscr{W}) \cong \lambda_{0} D K(\mathscr{W}), \quad \mathcal{D}_{*}\left({ }^{s} \mathscr{T}_{\infty}\right) \cong \lambda_{0} K\left({ }^{s} \mathscr{T}_{\infty}\right), \\
\mathcal{D}_{*}\left({ }^{d} \mathscr{T}\right) \cong \lambda_{0} K\left({ }^{d} \mathscr{T}\right), & \mathcal{D}_{*}\left({ }^{v} \mathscr{T}\right) \cong \lambda_{0} K\left({ }^{v} \mathscr{T}\right) .
\end{array}
$$

This follows from Theorem 4.5.2 and Examples 4.1.3 and 4.5.1.

The stable quadratic module $\mathcal{D}_{*}\left({ }^{b} \mathscr{T}\right)$ is not related to any $K$-theory spectrum. Actually, Breuning defines the $K$-theory of a triangulated category in dimensions $i=0,1$ as

$$
K_{i}\left({ }^{b} \mathscr{T}\right)=\pi_{i} \mathcal{D}_{*}\left({ }^{b} \mathscr{T}\right)
$$

1.7. Comparison morphisms. There are several comparison morphisms between the $K$-theories which appeared in the introduction. In this section we recover these morphisms in dimensions $i=0,1$ from certain explicit morphisms between the stable quadratic modules of Definition 1.5.1 that calculate, by Theorem 1.6.3, the 1 -types of the $K$-theory spectra. For more explicit descriptions of the maps of spectra involved, and of the spectra themselves, we refer the reader to Subsection 4.5.

For a Waldhausen category $\mathscr{W}$, we have honest and derived determinant functors, see Definitions 1.2.3 and 1.2.11. A derived determinant functor $\operatorname{det}^{\prime}: \mathscr{W} \rightarrow \mathscr{P}$ yields an honest determinant functor, defined by precomposition with the functor sending a weak equivalence in $\mathscr{W}$ to its corresponding isomorphism in the homotopy category Ho $\mathscr{W}$,

$$
\operatorname{det}: \operatorname{we}(\mathscr{W}) \longrightarrow \operatorname{iso}(\mathrm{Ho} \mathscr{W}) \stackrel{\operatorname{det}^{\prime}}{\longrightarrow} \mathscr{P} \text {. }
$$


Additivity data for det are defined as for det $^{\prime}$. This gives rise to a morphism of stable quadratic modules defined on generators by,

$$
\begin{aligned}
& \mathcal{D}_{*}(\mathscr{W}) \longrightarrow \mathcal{D}_{*}^{\text {der }}(\mathscr{W}), \\
& {[X] \longmapsto[X], } \\
& {\left[f: X \stackrel{\sim}{\longrightarrow} X^{\prime}\right] \longmapsto\left[\{f\}: X \stackrel{\cong}{\longrightarrow} X^{\prime}\right], } \\
& {[\Delta] \longmapsto[\Delta] . }
\end{aligned}
$$

Here $X$ is an object of $\mathscr{W},\{f\}$ denotes the homotopy class of a weak equivalence $f$ in $\mathscr{W}$, and $\Delta$ is a cofiber sequence. This morphism was shown in $[\mathbf{M u r}]$ to be an isomorphism, hence

$$
K_{0}(\mathscr{W}) \cong D K_{0}(\mathscr{W}), \quad K_{1}(\mathscr{W}) \cong D K_{1}(\mathscr{W})
$$

Let $\mathscr{T}$ be a triangulated category. Special octahedra are also ordinary, and distinguished triangles and ordinary octahedra are also virtual. Hence, restricting additivity data, any virtual determinant functor produces a Breuning determinant functor, and any Breuning determinant functor yields a special determinant functor. This gives rise to obvious morphisms of stable quadratic modules defined by the aforementioned inclusions of sets of generators,

$$
\mathcal{D}_{*}\left({ }^{d} \mathscr{T}\right) \longrightarrow \mathcal{D}_{*}\left({ }^{b} \mathscr{T}\right) \longrightarrow \mathcal{D}_{*}\left({ }^{v} \mathscr{T}\right) .
$$

These morphisms are the identity in degree 0 . They can be easily shown to be isomorphisms on $\pi_{0}$, see [Nee2, Bre1],

$$
K_{0}\left({ }^{d} \mathscr{T}\right) \cong K_{0}\left({ }^{b} \mathscr{T}\right) \cong K_{0}\left({ }^{v} \mathscr{T}\right) .
$$

Moreover, the first one is surjective in degree 1 , since $\mathcal{D}_{*}\left({ }^{d} \mathscr{T}\right)$ and $\mathcal{D}_{*}\left({ }^{b} \mathscr{T}\right)$ have the same generators, but the latter has more relations than the former, corresponding to non-special octahedra. In particular, the first morphism induces an epimorphism in $\pi_{1}$,

$$
K_{1}\left({ }^{d} \mathscr{T}\right) \longrightarrow K_{1}\left({ }^{b} \mathscr{T}\right) .
$$

We do not know of any example where this morphism has a non-trivial kernel. This may be due to our lack of knowledge about non-special octahedra.

Let $\mathscr{T}$ be a triangulated category with a $t$-structure with heart $\mathscr{A}$. Exact sequences in $\mathscr{A}$ extend uniquely to exact triangles in $\mathscr{T}$, see Subsection 2.4, and staircase diagrams extend uniquely to octahedra, which are therefore special, see Remark 1.3.4. Hence, a special determinant 
functor on $\mathscr{T}$ restricts to a determinant functor on $\mathscr{A}$. This defines an obvious stable quadratic module morphism

$$
\mathcal{D}_{*}(\mathscr{A}) \longrightarrow \mathcal{D}_{*}\left({ }^{d} \mathscr{T}\right) .
$$

Theorem 2.4.12 below shows that, if the $t$-structure is bounded and nondegenerate, this morphism and the two morphisms in (1.7.2) are weak equivalences. In particular,

$$
K_{i}(\mathscr{A}) \cong K_{i}\left({ }^{d} \mathscr{T}\right) \cong K_{i}\left({ }^{b} \mathscr{T}\right) \cong K_{i}\left({ }^{v} \mathscr{T}\right), \quad i=0,1 .
$$

Actually, the morphisms in (1.7.2) are isomorphisms by the five lemma, since they are the identity in degree 0 . The isomorphism $K_{1}(\mathscr{A}) \cong$ $K_{1}\left({ }^{b} \mathscr{T}\right)$ was previously obtained by Breuning [Bre1, Corollary 5.3] using different methods.

Similarly, if $\mathscr{E}$ is an exact category, a determinant functor on the strongly triangulated category $D^{b}(\mathscr{E})$ 'restricts' to a Deligne determinant functor on the Waldhausen category $C^{b}(\mathscr{E})$ along the canonical functor $C^{b}(\mathscr{E}) \rightarrow D^{b}(\mathscr{E})$, and also on $\mathscr{E}$, regarded as complexes concentrated in degree 0 . Hence we have stable quadratic module morphisms

$$
\mathcal{D}_{*}(\mathscr{E}) \longrightarrow \mathcal{D}_{*}\left(C^{b}(\mathscr{E})\right) \longrightarrow \mathcal{D}_{*}\left({ }^{s} D^{b}(\mathscr{E})\right) \text {. }
$$

The second morphism here is the identity in degree 0 . It is surjective in degree 1 , since any distinguished triangle in $D^{b}(\mathscr{T})$ is isomorphic to a distinguished triangle coming from a cofiber sequence in $C^{b}(\mathscr{E})$, and any isomorphism in $D^{b}(\mathscr{E})$ can be represented by a zigzag of weak equivalences in $C^{b}(\mathscr{E})$. Hence, we obtain an isomorphism in $\pi_{0}$ and a surjection in $\pi_{1}$,

$$
K_{0}\left(C^{b}(\mathscr{E})\right) \cong K_{0}\left({ }^{s} D^{b}(\mathscr{E})\right), \quad K_{1}\left(C^{b}(\mathscr{E})\right) \longrightarrow K_{1}\left({ }^{s} D^{b}(\mathscr{E})\right)
$$

The first morphism in (1.7.4) is a weak equivalence by the following theorem and [Cis1], actually

$$
K_{i}(\mathscr{E}) \cong K_{i}\left(C^{b}(\mathscr{E})\right), \quad i \geq 0 .
$$

Theorem 1.7.5. The images under the functor $\lambda_{0}$ of Lemma 1.6.1 of the maps between $K$-theory spectra

$$
\begin{gathered}
K(\mathscr{W}) \longrightarrow D K(\mathscr{W}), \quad K\left({ }^{d} \mathscr{T}\right) \longrightarrow K(v \mathscr{T}), K(\mathscr{A}) \longrightarrow K\left({ }^{d} \mathscr{T}\right), \\
K(\mathscr{E}) \stackrel{\sim}{\longrightarrow} K\left(C^{b}(\mathscr{E})\right), \quad K\left(C^{b}(\mathscr{E})\right) \longrightarrow\left(^{s} D^{b}(\mathscr{E})\right),
\end{gathered}
$$

of Examples 4.5.3(1), (2), (3), (7), and (8), respectively, coincide with the stable quadratic module morphisms given in (1.7.1), the composite of (1.7.2), and (1.7.3) and (1.7.4).

This follows from Theorem 4.5.2 and Example 4.5.3. 
There is yet another comparison morphism of stable quadratic modules which is not related to spectra, since it is connected to Breuning determinant functors. Let $\mathscr{T}_{3}$ be a 3 -pretriangulated category. Distinguished octahedra are ordinary octahedra in the underlying triangulated structure. Therefore, a Breuning determinant functor on the triangulated category underlying $\mathscr{T}_{3}$ yields a determinant functor on $\mathscr{T}_{3}$ in the sense of Definition 1.3.10. This gives rise to a stable quadratic module morphism

$$
\mathcal{D}_{*}\left({ }^{s} \mathscr{T}_{3}\right) \longrightarrow \mathcal{D}_{*}\left({ }^{b} \mathscr{T}_{3}\right),
$$

which is the identity on generators, but the target has more relations than the source, corresponding to non-distinguished octahedra. It is actually the identity in degree 0 and surjective in degree 1 . In particular, we obtain an isomorphism on $\pi_{0}$ and a surjection on $\pi_{1}$,

$$
K_{0}\left({ }^{s} \mathscr{T}_{3}\right) \cong K_{0}\left({ }^{b} \mathscr{T}_{3}\right), \quad K_{1}\left({ }^{s} \mathscr{T}_{3}\right) \longrightarrow K_{1}\left({ }^{b} \mathscr{T}_{3}\right) .
$$

\section{Applications}

2.1. Derived and non-derived determinant functors on a Waldhausen category. Grothendieck asked in a letter to Knudsen whether determinant functors on an additive or abelian category $\mathscr{E}$ coincide essentially with determinant functors in the bounded derived category $D^{b}(\mathscr{E})$ regarded as triangulated category equipped with a 'category of true triangles' [Knu1, Appendix B]. We extend the question to exact categories. We interpret the 'category of true triangles' to be the bounded derived category of the exact category $S_{2}(\mathscr{E})$ of short exact sequences in $\mathscr{E}$, which coincides with the homotopy category of the Waldhausen category $S_{2} C^{b}(\mathscr{E})$ of short exact sequences of bounded complexes in $\mathscr{E}$. With this interpretation, determinant functors in the triangulated category equipped with a 'category of true triangles' are derived determinant functors in $C^{b}(\mathscr{E})$.

The Waldhausen category $C^{b}(\mathscr{E})$ has cylinders and a saturated class of weak equivalences, therefore the two following results answer Grothendieck's question and its generalization positively.

Corollary 2.1.1. If we regard $\mathscr{E}$ as the full subcategory of complexes in $C^{b}(\mathscr{E})$ concentrated in degree 0 , then any determinant functor on $\mathscr{E}$ factors through a determinant functor in $C^{b}(\mathscr{E})$ in an essentially unique way.

This follows from Theorems 1.5.4 and 1.6.3 and from the GilletWaldhausen theorem [Cis1]. A direct proof of this result can be found in $[\mathbf{K n u 1 ]}$. 
Corollary 2.1.2. Let $\mathscr{W}$ be a Waldhausen category with cylinders and a saturated class of weak equivalences, i.e. weak equivalences are exactly those maps in $\mathscr{W}$ which become invertible in Ho $\mathscr{W}$. Then any determinant functor on $\mathscr{W}$ factors through a derived determinant functor in an essentially unique way.

This follows from Theorem 1.5.4 and [Mur, Theorem 6.1].

Remark 2.1.3. If the class of weak equivalences in $\mathscr{W}$ is not saturated then Weiss's Whitehead group $\mathrm{Wh}(\mathscr{W})$ may not vanish [Wei], and in this case the universal determinant functor det: $\mathscr{W} \rightarrow \mathcal{D}_{*}(\mathscr{W})$ need not factor through a derived determinant functor, compare [Mur, Remark 6.3].

2.2. Generators and (some) relations for $\boldsymbol{K}_{\mathbf{1}}$. Nenashev [Nen] considered pairs of short exact sequences over the same objects in an exact category $\mathscr{E}$,

$$
X \underset{f^{\prime}}{\stackrel{f}{\rightleftarrows}} Y \underset{p^{\prime}}{\stackrel{p}{\rightleftarrows}} C .
$$

Such a pair yields an element in $K_{1}(\mathscr{E})$. Nenashev proved that any element in $K_{1}(\mathscr{E})$ is of this kind and computed a set of relations among them, associated to $3 \times 3$ diagrams, yielding a presentation of $K_{1}(\mathscr{E})$.

Vaknin [Vak2] considered pairs of distinguished triangles over the same objects in a triangulated category $\mathscr{T}$,

$$
X \underset{f^{\prime}}{\stackrel{f}{\longrightarrow}} Y \underset{i^{\prime}}{\stackrel{i}{\longrightarrow}} Z \underset{q^{\prime}}{\stackrel{q}{\longrightarrow}} \Sigma X .
$$

Using similar techniques, Vaknin [Vak2] proved that any element in Neeman's $K_{1}\left({ }^{d} \mathscr{T}\right)$ is of this kind and computed a set of relations among them, extending Nenashev's, yielding a presentation of $K_{1}(d \mathscr{T})$, as in the exact case, see Proposition 2.2.8.

Muro and Tonks considered in [MT2] diagrams in a Waldhausen category $\mathscr{W}$,

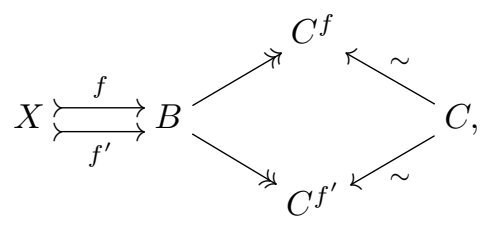

consisting of two cofiber sequences and two weak equivalences. They extended Nenashev's results, showing that any element in $K_{1}(\mathscr{W})$ is of this 
kind and computing a set of relations among them generalizing Nenashev's. Some evidence was given for the conjecture that these relations define a presentation of $K_{1}(\mathscr{W})$.

In this section we indicate how these results extend to Breuning's $K_{1}\left({ }^{b} \mathscr{T}\right)$, Neeman's $K_{1}\left({ }^{v} \mathscr{T}\right)$, and Maltsiniotis's $K_{1}\left({ }^{s} \mathscr{T}_{\infty}\right)$.

Let $=b, d, v$ or $s$. Denote simply by $\mathscr{T}$ a triangulated category, or a strongly triangulated category if $=s$. A -triangle is a distinguished triangle if $\bullet=b, d, s$ and a virtual triangle if $\bullet=v$. Given a pair of -triangles (2.2.2) we define

$$
\begin{aligned}
{\left[X \underset{f^{\prime}}{\stackrel{f}{\longrightarrow}} Y \underset{i^{\prime}}{\stackrel{i}{\longrightarrow}} Z \underset{q^{\prime}}{\stackrel{q}{\longrightarrow}} \Sigma X\right]=} & -[X \stackrel{f}{\longrightarrow} Y \stackrel{i}{\longrightarrow} Z \stackrel{q}{\longrightarrow} \Sigma X] \\
& +\left[X \stackrel{f^{\prime}}{\longrightarrow} Y \stackrel{i^{\prime}}{\longrightarrow} Z \stackrel{q^{\prime}}{\longrightarrow} \Sigma X\right] \in \mathcal{D}_{*}(\bullet \mathscr{T}) .
\end{aligned}
$$

Notice that this element is in the kernel of $\partial$, i.e.

$$
\left[X \underset{f^{\prime}}{\stackrel{f}{\longrightarrow}} Y \underset{i^{\prime}}{\stackrel{i}{\longrightarrow}} Z \underset{q^{\prime}}{\stackrel{q}{\longrightarrow}} \Sigma X\right] \in \pi_{1} \mathcal{D}_{*}(\bullet \mathscr{T}) \cong K_{1}(\bullet \mathscr{T}) .
$$

Moreover, a pair given by twice the same $\bullet$-triangle is zero

$$
[X \underset{f}{\stackrel{f}{\longrightarrow}} Y \underset{i}{\stackrel{i}{\longrightarrow}} Z \underset{q}{\stackrel{q}{\longrightarrow}} \Sigma X]=0 .
$$

Theorem 2.2.4. Any element in $K_{1}(\bullet \mathscr{T})$ is represented by a pair of -triangles.

This result follows from Theorem 4.6.2.

Definition 2.2.5. A $3 \times 3$ diagram in $\mathscr{T}$ is a diagram
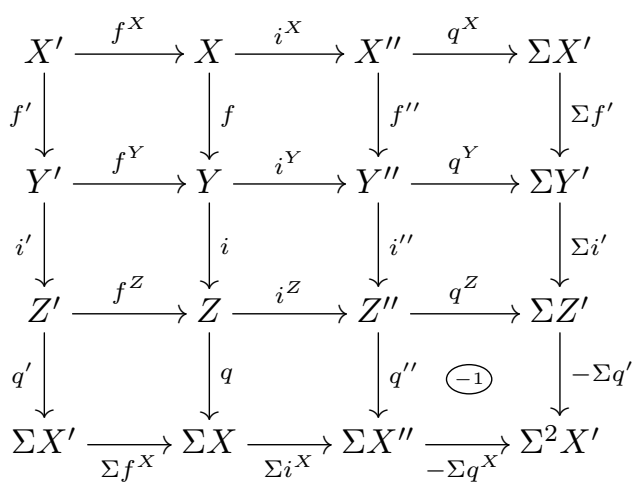
where all squares commute except for the bottom right square, which is anticommutative, $\left(\Sigma q^{\prime}\right) q^{Z}+\left(\Sigma q^{X}\right) q^{\prime \prime}=0$. Moreover, all rows and columns are -triangles.

A - octahedron is an ordinary octahedron if $\bullet=b$, a special octahedron if $\bullet=d$, a distinguished octahedron if $\bullet=s$, and a virtual octahedron if $=v$.

A $3 \times 3$ diagram is $\bullet$-coherent if there exist four $\bullet$-octahedra
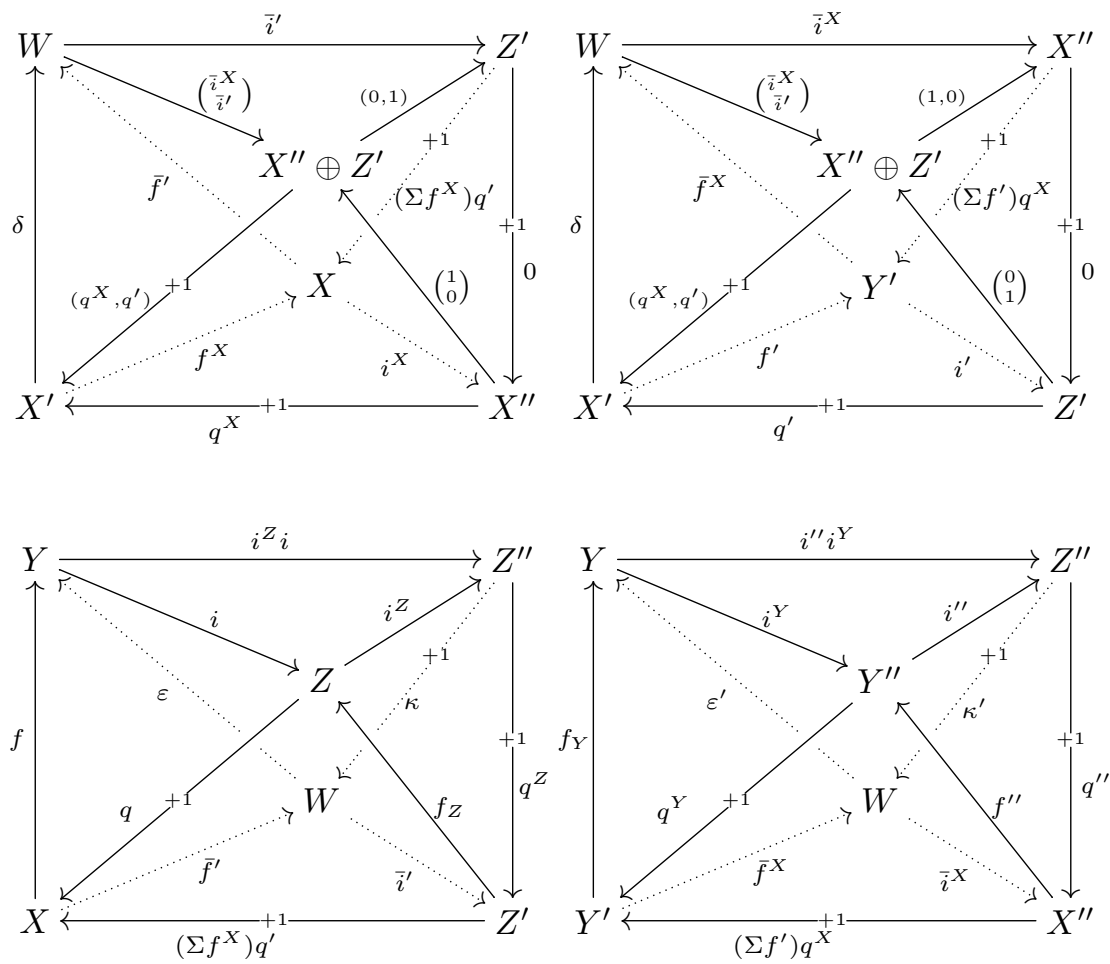

such that in the two last octahedra $\varepsilon=\varepsilon^{\prime}$ and $\kappa=\kappa^{\prime}$.

This kind of diagram has previously appeared in [Vak2, KN].

Remark 2.2.7. As Vaknin pointed out in [Vak2, Remark 5.2], if we start with a $3 \times 3$ diagram of distinguished triangles as in (2.2.6) it is always possible to construct four octahedra as above, but, in general, one cannot guarantee $\varepsilon=\varepsilon^{\prime}$ or $\kappa=\kappa^{\prime}$, i.e. the third and fourth octahedra may contain different distinguished triangle completions of $i^{Z} i=i^{\prime \prime} i^{Y}: Y \rightarrow$ $Z^{\prime \prime}$. The procedure is as follows: 
(1) Extend $\left(q^{X}, q^{\prime}\right)$ to an exact triangle. This produces $\bar{i}^{X}, \bar{i}^{\prime}$, and $\delta$. All morphisms in the first octahedron are now defined, except for $\bar{f}^{\prime}$. The octahedral axiom yields a morphism $\bar{f}^{\prime}$ such that the resulting diagram is an octahedron. This octahedron is always special since we have the following triangle isomorphisms where the bottom triangle is distinguished, being the direct sum of two distinguished triangles,
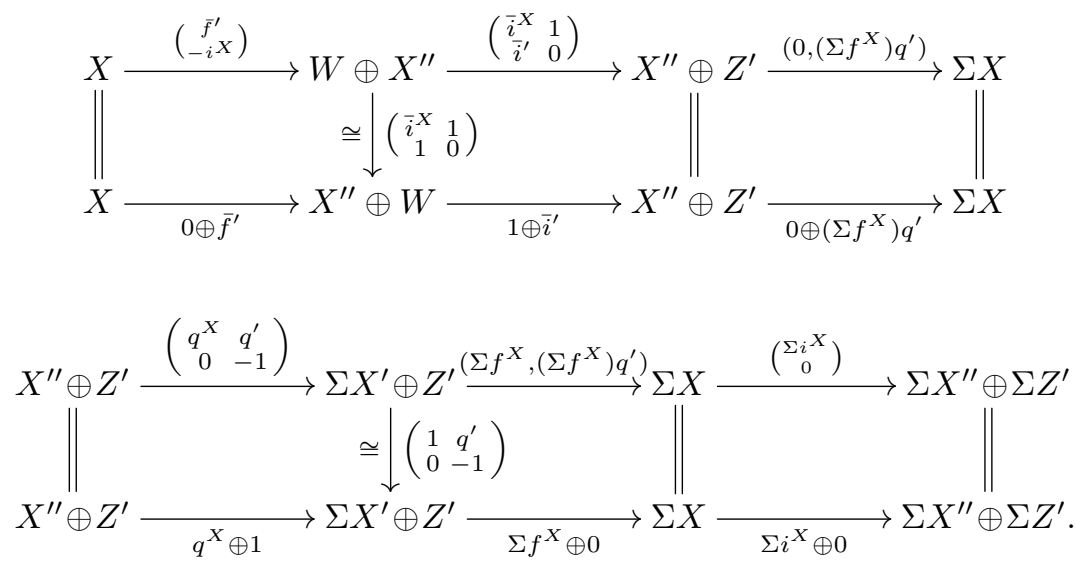

(2) All morphisms in the second octahedron are defined, except for $\bar{f}^{X}$. Apply the octahedral axiom to obtain a morphism $\bar{f}^{X}$ such that the resulting diagram is an octahedron. This octahedron is also always special since we have the following triangle isomorphisms with distinguished bottom part,
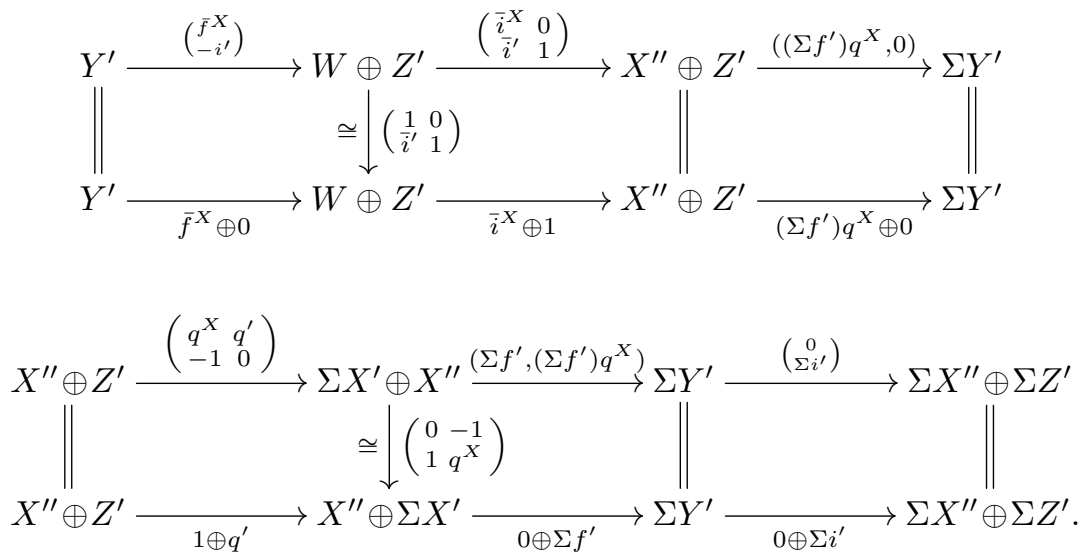
(3) The morphisms $\varepsilon$ and $\kappa$ in the third octahedron are obtained by applying the octahedral axiom, the rest has been previously defined. Similarly $\varepsilon^{\prime}$ and $\kappa^{\prime}$ in the fourth octahedron. Nothing guarantees that these octahedra are special, nor that $\varepsilon=\varepsilon^{\prime}$ and $\kappa=\kappa^{\prime}$.

The following result extends Vaknin's relations to $=b, s, v$, but we do not prove their sufficiency in these contexts.

Proposition 2.2.8. Suppose we have two $\bullet$-coherent $3 \times 3$ diagrams over the same objects, $j=1,2$,

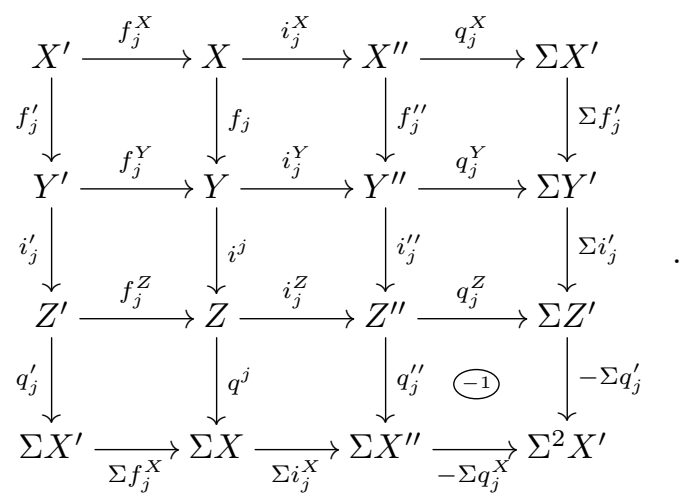

Then the following equation holds in $K_{1}(\bullet \mathscr{T})$,

$$
\begin{aligned}
& {\left[X^{\prime} \underset{f_{2}^{X}}{\stackrel{f_{1}^{X}}{\longrightarrow}} X \underset{i_{2}^{X}}{\stackrel{i_{1}^{X}}{\longrightarrow}} X^{\prime \prime} \underset{q_{2}^{X}}{\stackrel{q_{1}^{X}}{\longrightarrow}} \Sigma X^{\prime}\right]-\left[Y^{\prime} \underset{f_{2}^{Y}}{\stackrel{f_{1}^{Y}}{\longrightarrow}} Y \underset{i_{2}^{Y}}{\stackrel{i_{1}^{Y}}{\longrightarrow}} Y^{\prime \prime} \underset{q_{2}^{Y}}{\stackrel{q_{1}^{Y}}{\longrightarrow}} \Sigma Y^{\prime}\right]} \\
& +\left[Z^{\prime} \underset{f_{2}^{Z}}{\stackrel{f_{1}^{Z}}{\longrightarrow}} Z \underset{i_{2}^{Z}}{\stackrel{i_{1}^{Z}}{\longrightarrow}} Z^{\prime \prime} \underset{q_{2}^{Z}}{\stackrel{q_{1}^{Z}}{\longrightarrow}} \Sigma Y^{\prime}\right] \\
& =\left[X^{\prime} \underset{f_{2}^{\prime}}{\stackrel{f_{1}^{\prime}}{\longrightarrow}} Y^{\prime} \underset{i_{2}^{\prime}}{\stackrel{i_{1}^{\prime}}{\longrightarrow}} Z^{\prime} \underset{q_{2}^{\prime}}{\stackrel{q_{1}^{\prime}}{\longrightarrow}} \Sigma X^{\prime}\right]-\left[X \underset{f_{2}}{\stackrel{f_{1}}{\longrightarrow}} Y \underset{i_{2}}{\stackrel{i_{1}}{\longrightarrow}} Z \underset{q_{2}}{\stackrel{q_{1}}{\longrightarrow}} \Sigma X\right] \\
& +\left[X^{\prime \prime} \underset{f_{2}^{\prime \prime}}{\stackrel{f_{1}^{\prime \prime}}{\longrightarrow}} Y^{\prime \prime} \underset{i_{2}^{\prime \prime}}{\stackrel{i_{1}^{\prime \prime}}{\longrightarrow}} Z^{\prime \prime} \underset{q_{2}^{\prime \prime}}{\stackrel{q_{1}^{\prime \prime}}{\longrightarrow}} \Sigma X^{\prime \prime}\right] .
\end{aligned}
$$

This result follows from Corollary 4.6.6.

\subsection{On additivity for low-dimensional $K$-theory of triangu-} lated categories. In this section we prove an additivity theorem for low-dimensional $K$-theories of (strongly) triangulated categories. 
Theorem 2.3.1 (Additivity). Let $F, G, H: \mathscr{T} \rightarrow \mathscr{T}^{\prime}$ be exact functors between (strongly) triangulated categories. Denote $\bullet=b, d$, or $v$ in the triangulated case and $=s$ in the strongly triangulated case. Suppose we have a natural $\bullet$-triangle,

$$
F(X) \stackrel{f(X)}{\longrightarrow} G(X) \stackrel{i(X)}{\longrightarrow} H(X) \stackrel{q(X)}{\longrightarrow} \Sigma F(X)
$$

such that for any $\bullet$-triangle $X \stackrel{f}{\rightarrow} Y \stackrel{i}{\rightarrow} Z \stackrel{q}{\rightarrow} \Sigma X$, the $3 \times 3$ diagram

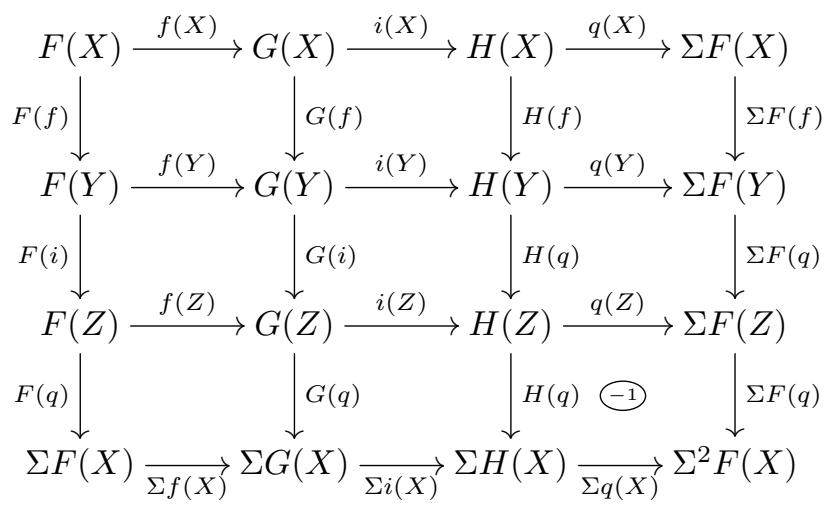

is - coherent. Then

$$
K_{i}(\bullet F)+K_{i}(\bullet H)=K_{i}\left({ }^{\bullet} G\right): K_{i}(\bullet \mathscr{T}) \longrightarrow K_{i}\left(\mathscr{T}^{\prime}\right), \quad i=0,1
$$

Proof: For $K_{0}$ the result follows from the following equation,

$$
\begin{aligned}
\partial[F(X) \stackrel{f(X)}{\longrightarrow} G(X) \stackrel{i(X)}{\longrightarrow} H(X) \stackrel{q(X)}{\longrightarrow} \Sigma F(X)] \\
=-[G(X)]+[H(X)]+[F(X)] .
\end{aligned}
$$

Any element in $K_{1}$ is represented by a pair of -triangles,

$$
\left[X \underset{f^{\prime}}{\stackrel{f}{\longrightarrow}} Y \underset{i^{\prime}}{\stackrel{i}{\longrightarrow}} Z \underset{q^{\prime}}{\stackrel{q}{\longrightarrow}} \Sigma X\right]
$$


see Theorem 2.2.4. If we apply Proposition 2.2.8 to the induced -coherent $3 \times 3$ diagrams $(2.3 .3)$ we obtain the following equation in $K_{1}$,

$$
\begin{aligned}
& 0=[F(X) \underset{f(X)}{\stackrel{f(X)}{\longrightarrow}} G(X) \underset{i(X)}{\stackrel{i(X)}{\longrightarrow}} H(X) \underset{q(X)}{\stackrel{q(X)}{\longrightarrow}} \Sigma F(X)] \\
& -[F(Y) \underset{f(Y)}{\stackrel{f(Y)}{\longrightarrow}} G(Y) \underset{i(Y)}{\stackrel{i(Y)}{\longrightarrow}} H(Y) \underset{q(Y)}{\stackrel{q(Y)}{\longrightarrow}} \Sigma F(Y)] \\
& +[F(Z) \underset{f(Z)}{\stackrel{f(Z)}{\longrightarrow}} G(Z) \underset{i(Z)}{\stackrel{i(Z)}{\longrightarrow}} H(Z) \underset{q(Z)}{\stackrel{q(Z)}{\longrightarrow}} \Sigma F(Z)] \\
& =\left[F(X) \underset{G\left(f^{\prime}\right)}{\stackrel{F(f)}{\longrightarrow}} F(Y) \underset{F\left(i^{\prime}\right)}{\stackrel{F(i)}{\longrightarrow}} F(X) \underset{F\left(q^{\prime}\right)}{\stackrel{F(q)}{\longrightarrow}} \Sigma F(X)\right] \\
& -\left[G(X) \underset{G\left(f^{\prime}\right)}{\stackrel{G(f)}{\longrightarrow}} G(Y) \underset{G\left(i^{\prime}\right)}{\stackrel{G(i)}{\longrightarrow}} G(Z) \underset{G\left(q^{\prime}\right)}{\stackrel{G(q)}{\longrightarrow}} \Sigma G(X)\right] \\
& +\left[H(X) \underset{G\left(f^{\prime}\right)}{\stackrel{H(f)}{\longrightarrow}} H(Y) \underset{H\left(i^{\prime}\right)}{\stackrel{H(i)}{\longrightarrow}} H(Z) \underset{H\left(q^{\prime}\right)}{\stackrel{H(q)}{\longrightarrow}} \Sigma H(X)\right],
\end{aligned}
$$

hence we are done.

Notice that this additivity theorem does not contradict [Sch, Remark 2.3].

Remark 2.3.4. The hypotheses are satisfied if the natural distinguished triangle has a model, e.g. if $\mathscr{T}$ and $\mathscr{T}^{\prime}$ are categories of perfect complexes over two rings $R$ and $R^{\prime}$, and the exact functors $F, G$, and $H$ are given by the derived tensor product with perfect complexes of $R^{\prime}$ - $R$-bimodules $F_{*}, G_{*}$, and $H_{*}$ fitting into a -triangle $F_{*} \rightarrow G_{*} \rightarrow H_{*} \rightarrow \Sigma F_{*}$.

\subsection{Low-dimensional $K$-theory of a triangulated category with} a $t$-structure. A $t$-structure in a triangulated category $\mathscr{T}$ consists essentially of a full abelian subcategory $\mathscr{A} \subset \mathscr{T}$, called core or heart, and a splitting of the inclusion given by a cohomological functor

$$
H^{0}: \mathscr{T} \longrightarrow \mathscr{A} \text {. }
$$

Some axioms modelled on the canonical example $\mathscr{T}=D^{b}(\mathscr{A})$ must be satisfied, see [GM, IV.4.2 and IV.4.11]. The cohomology of an object $X$ 
in $\mathscr{T}$ is defined as

$$
H^{n} X=H^{0} \Sigma^{n} X, \quad n \in \mathbb{Z} .
$$

In this section we consider $t$-structures satisfying the following additional properties, which hold in the canonical example but which are often relaxed. Our $t$-structures will be non-degenerate, i.e. $H^{*} X=0$ iff $X=0$, and bounded, i.e. the graded object $H^{*} X$ must be bounded for any $X$.

Any object $X$ in $\mathscr{T}$ fits into a functorial distinguished triangle,

$$
\Delta_{X}: X_{\leq-1} \longrightarrow X \longrightarrow X_{\geq 0} \longrightarrow \Sigma X_{\leq-1},
$$

where, as the notation suggests, the homology of $X_{\leq-1}$ (resp. $X_{\geq 0}$ ) is concentrated in negative (resp. non-negative) degrees. In particular, the first (resp. second) arrow induces an isomorphism in $H^{n}$ for all $n<0$ (resp. $n \geq 0$ ).

An object $X$ in $\mathscr{T}$ is connective if $H^{n} X=0$ for $n>0$. For a connective object $X$, the distinguished triangle $\Delta_{X}$ looks like

$$
\Delta_{X}: X_{\leq-1} \longrightarrow X \longrightarrow H^{0} X \longrightarrow \Sigma X_{\leq-1} .
$$

Since the our $t$-structures are bounded, all objects become connective after suspending a finite number of times.

A short exact sequence in $\mathscr{A}$,

$$
\Delta: A \stackrel{i}{\longrightarrow} B \stackrel{q}{\longrightarrow} C,
$$

extends uniquely to a distinguished triangle, that we denote in the same way,

$$
\Delta: A \stackrel{i}{\longrightarrow} B \stackrel{q}{\longrightarrow} C \stackrel{\epsilon}{\longrightarrow} \Sigma A .
$$

This defines a natural isomorphism

$$
\operatorname{Ext}_{\mathscr{A}}^{1}(C, A) \cong \mathscr{T}(C, \Sigma A),
$$

and moreover, a morphism of stable quadratic modules, $\bullet=b, d, v$,

$$
j: \mathcal{D}_{*}(\mathscr{A}) \longrightarrow \mathcal{D}_{*}(\bullet \mathscr{T}) .
$$

Lemma 2.4.1. The stable quadratic module morphism $j$ is levelwise injective.

Proof: The morphism is obviously injective in degree 0 , since it is given by an inclusion of the basis of $\mathcal{D}_{0}(\mathscr{A})$ (the non-trivial objects of $\mathscr{A}$ ) into the basis of $\mathcal{D}_{0}(\bullet \mathscr{T})$ (the non-trivial objects of $\mathscr{T}$ ). An easy diagram chase shows that $j$ is injective in degree 1 if and only if $\pi_{1} j$ is injective. The latter is true. Actually, the comparison morphism $K_{n}(\mathscr{A}) \rightarrow K_{n}(\bullet \mathscr{T})$ is split injective for any $n \geq 0, \bullet=d, v$, see [Nee2, 
Theorem 50 (i)], and $\pi_{1} j$ is the case $n=1$, see Theorem 1.7.5. The case $=b$ then follows from the factorization (1.7.2). Alternatively, the cases $\bullet=b, d$ also follow from [Bre1, Corollary 5.3]. It is also possible to give a unified direct proof, not invoking Neeman's or Breuning's results, see Remark 2.4.21 below.

For any object $X$ in $\mathscr{T}$, consider the distinguished triangle

$$
\Gamma_{X}: X \longrightarrow 0 \longrightarrow \Sigma X \stackrel{1}{\longrightarrow} \Sigma X
$$

Proposition 2.4.2. The stable quadratic module $\mathcal{D}_{*}(\bullet \mathscr{T}), \bullet=b, d, v$, is generated by the image of the generators (G1) and (G3) of $\mathcal{D}_{*}(\mathscr{A})$ by $j$ together with the generators $\left[\Gamma_{X}\right]$ and $\left[\Delta_{Y}\right]$, where $X$ runs over all objects of $\mathscr{T}$ and $Y$ runs over all connective objects.

This follows from Lemmas 2.4.3, 2.4.6, and 2.4.8 below, by induction on the interval where the cohomology of a given object (or the cohomology of the three objects in a given -triangle) is concentrated.

The following lemma is simply an application of (R2).

Lemma 2.4.3. Given objects $X$ and $Y$ in $\mathscr{T}$, with $Y$ connective, the following formulas hold in $\mathcal{D}_{0}(\bullet \mathscr{T}), \bullet=b, d, v$ :

$$
\partial\left[\Gamma_{X}\right]=[\Sigma X]+[X], \quad[Y]+\partial\left[\Delta_{Y}\right]=j\left[H^{0} Y\right]+\left[Y_{\leq-1}\right] .
$$

Lemma 2.4.4. Let $\Delta$ in (1.3.1) be a-triangle where $X, Y$, and $C^{f}$ are connective, $=b, d, v$. There is a $\bullet$-octahedron

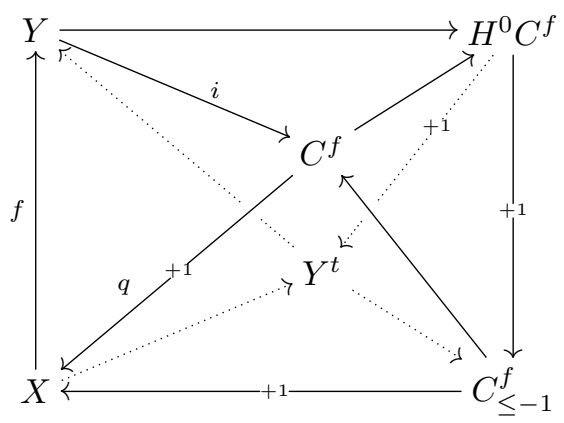

which is unique up to a unique isomorphism.

Proof: Everything is proven in [Vak3, Lemma 3.9 and Theorem 3.13], except that the octahedron is special for $\bullet=d$. This follows from Remark 1.3.4. 
The distinguished triangle on the bottom of the octahedron in the previous lemma,

$$
X \longrightarrow Y^{t} \longrightarrow C_{\leq-1}^{f} \longrightarrow \Sigma X,
$$

is known as the truncation of $\Delta$.

Lemma 2.4.6. In the conditions of the previous lemma, the following formula holds in $\mathcal{D}_{1}(\bullet \mathscr{T}), \bullet=b, d, v$ :

$$
\begin{aligned}
{\left[\Delta_{Y}\right]+j\left[\operatorname{Ker} H^{0} i^{f}\right.} & \left.\longmapsto H^{0} Y \longrightarrow H^{0} C^{f}\right]^{\left[Y_{\leq-1}\right]} \\
+\left[X \longrightarrow Y^{t} \longrightarrow C_{\leq-1}^{f}\right. & \longrightarrow \Sigma X] \\
& =\left[\Delta_{Y^{t}}\right]+[\Delta]+\left[\Delta_{C^{f}}\right]^{[X]} .
\end{aligned}
$$

Proof: Applying (R8) to the -octahedron in the previous lemma we obtain

$$
\begin{aligned}
{\left[Y^{t} \longrightarrow Y \longrightarrow H^{0} C^{f} \longrightarrow \Sigma Y^{t}\right]+\left[X \longrightarrow Y^{t} \longrightarrow\right.} & \left.C_{\geq 1}^{f} \longrightarrow \Sigma X\right] \\
& =[\Delta]+\left[\Delta_{C^{f}}\right]^{[X]} .
\end{aligned}
$$

By connectivity reasons, the morphism $Y_{\geq 1} \rightarrow Y$ factors uniquely through $Y^{t} \rightarrow Y$. The octahedral axiom yields an octahedron

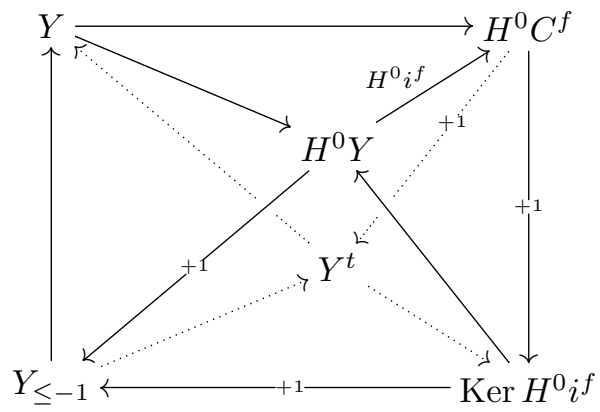

whose byproduct is necessarily the distinguished triangle associated to the short exact sequence $\operatorname{Ker} H^{0} i^{f} \longmapsto H^{0} Y \rightarrow H^{0} C^{f}$, since this short exact sequence is obtained by taking $H^{0}$ on $Y^{t} \rightarrow Y \rightarrow H^{0} C^{f} \rightarrow \Sigma Y^{t}$. This octahedron is special by Remark 1.3.4. Hence, by (R8),

$$
\begin{aligned}
{\left[\Delta_{Y^{t}}\right]+\left[Y^{t} \longrightarrow Y\right.} & \left.\longrightarrow H^{0} C^{f} \longrightarrow \Sigma Y^{t}\right] \\
& =\left[\Delta_{Y}\right]+j\left[\operatorname{Ker} H^{0} i^{f} \longleftrightarrow H^{0} Y \longrightarrow H^{0} C^{f}\right]^{\left[Y_{\leq-1}\right]} .
\end{aligned}
$$

The statement follows from the combination of the two previous formulas. 
The translation of a -triangle $\Delta$ as in (1.3.1) is the -triangle

$$
\Delta_{\mathrm{tr}}: Y \stackrel{i^{f}}{\longrightarrow} C^{f} \stackrel{-q^{f}}{\longrightarrow} \Sigma X \stackrel{\Sigma f}{\longrightarrow} \Sigma Y .
$$

Usually, the sign is placed in the third arrow, but both triangles are isomorphic. Actually, we could also place the sign in the first arrow.

Lemma 2.4.8. Given a-triangle $\Delta$ as in (1.3.1), the following formula holds in $\mathcal{D}_{1}(\bullet \mathscr{T})$ :

$$
\left[\Delta_{\mathrm{tr}}\right]+[\Delta]=\left[\Gamma_{X}\right]+\left\langle\left[C^{f}\right],[\Sigma X]\right\rangle .
$$

Proof: Consider the following $\bullet$-octahedron,

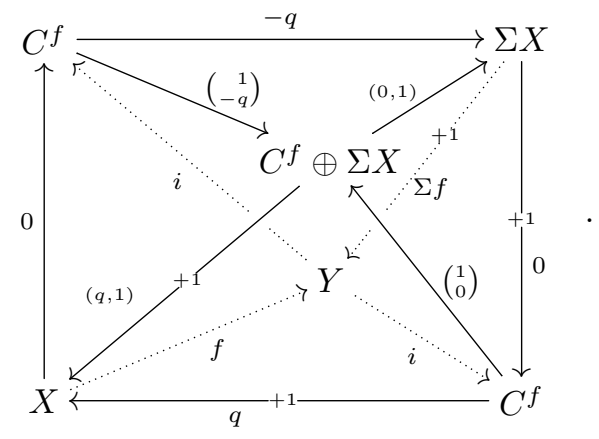

This octahedron is indeed special for $\bullet=d$, since we have the following isomorphisms where the lower rows are (direct sums of) distinguished triangles,
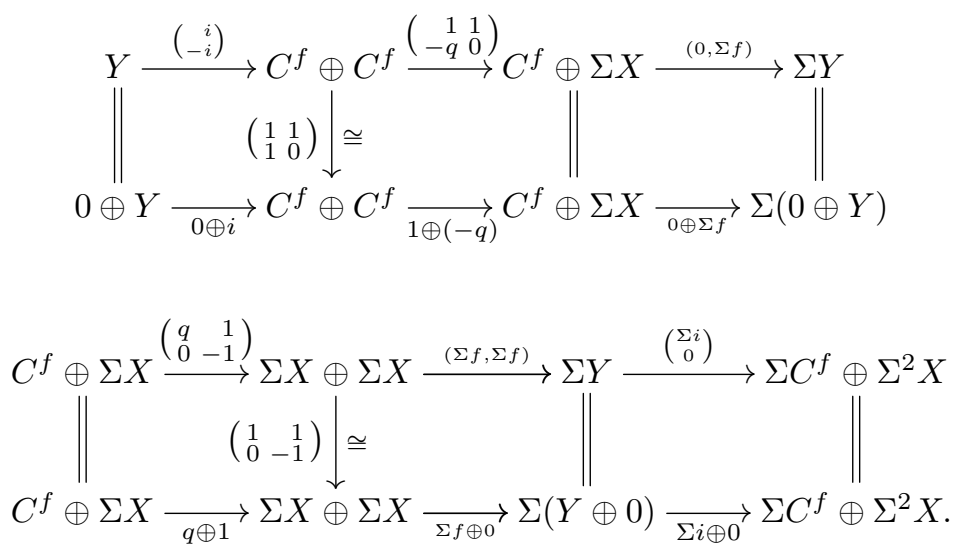
Relation (R8) yields the following equation,

$$
\begin{aligned}
{\left[\Delta_{\mathrm{tr}}\right]+[\Delta]=} & {\left[X \stackrel{0}{\longrightarrow} C^{f} \stackrel{\left(\begin{array}{c}
1 \\
-q
\end{array}\right)}{\longrightarrow} C^{f} \oplus \Sigma X \stackrel{(q, 1)}{\longrightarrow} \Sigma X\right] } \\
& +\left[C^{f} \stackrel{\left(\begin{array}{c}
1 \\
0
\end{array}\right)}{\longrightarrow} C^{f} \oplus \Sigma X \stackrel{(0,1)}{\longrightarrow} \Sigma X \stackrel{0}{\longrightarrow} \Sigma C^{f}\right] .
\end{aligned}
$$

Applying (R7) to the isomorphisms

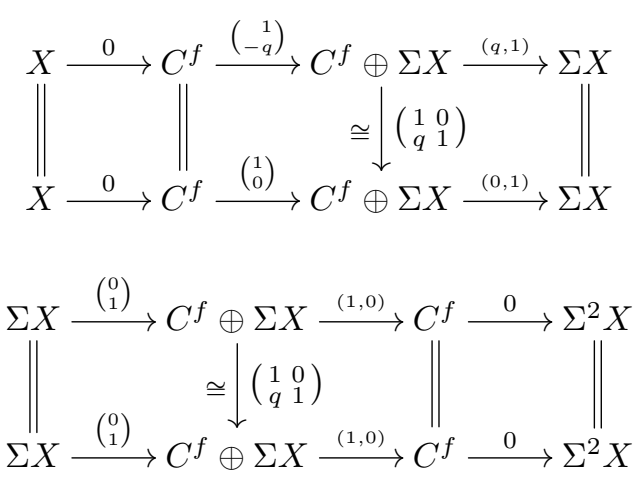

we obtain

$$
\begin{aligned}
{\left[X \stackrel{0}{\longrightarrow} C^{f} \stackrel{\left(\begin{array}{l}
1 \\
0
\end{array}\right)}{\longrightarrow} C^{f} \oplus \Sigma X \stackrel{(0,1)}{\longrightarrow} \Sigma X\right]+\left[\left(\begin{array}{cc}
1 & 0 \\
q & 1
\end{array}\right)\right] } \\
=\left[X \stackrel{0}{\longrightarrow} C^{f} \stackrel{\left(\begin{array}{c}
1 \\
-q
\end{array}\right)}{\longrightarrow} C^{f} \oplus \Sigma X \stackrel{(q, 1)}{\longrightarrow} \Sigma X\right], \\
{\left[\left(\begin{array}{ll}
1 & 0 \\
q & 1
\end{array}\right)\right]+\left[\Sigma X \stackrel{\left(\begin{array}{l}
0 \\
1
\end{array}\right)}{\longrightarrow} C^{f} \oplus \Sigma X \stackrel{(1,0)}{\longrightarrow} C^{f} \stackrel{0}{\longrightarrow} \Sigma X\right] } \\
=\left[\Sigma X \stackrel{\left(\begin{array}{l}
0 \\
1
\end{array}\right)}{\longrightarrow} C^{f} \oplus \Sigma X \stackrel{(1,0)}{\longrightarrow} C^{f} \stackrel{0}{\longrightarrow} \Sigma X\right] .
\end{aligned}
$$

In particular,

$$
\left[\left(\begin{array}{ll}
1 & 0 \\
q & 1
\end{array}\right)\right]=0
$$

$$
\begin{aligned}
{\left[X \stackrel{0}{\longrightarrow} C^{f} \stackrel{\left(\begin{array}{l}
1 \\
0
\end{array}\right)}{\longrightarrow} C^{f} \oplus \Sigma X \stackrel{(0,1)}{\longrightarrow} \Sigma X\right] } \\
\quad=\left[X \stackrel{0}{\longrightarrow} C^{f} \stackrel{\left(\begin{array}{c}
1 \\
-q
\end{array}\right)}{\longrightarrow} C^{f} \oplus \Sigma X \stackrel{(q, 1)}{\longrightarrow} \Sigma X\right] .
\end{aligned}
$$


Applying (R8) to the special octahedron

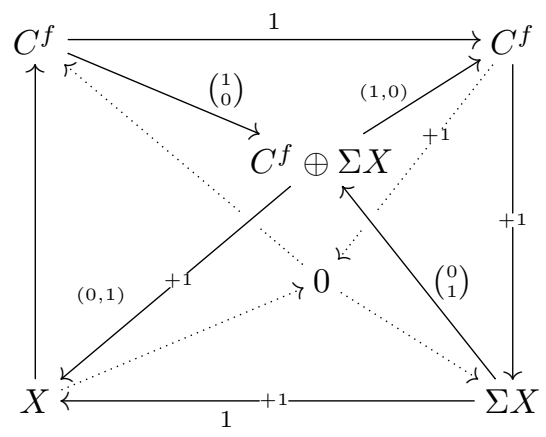

we obtain

$$
\begin{aligned}
{\left[\Gamma_{X}\right]=} & {\left[X \stackrel{0}{\longrightarrow} C^{f} \stackrel{\left(\begin{array}{l}
1 \\
0
\end{array}\right)}{\longrightarrow} C^{f} \oplus \Sigma X \stackrel{(0,1)}{\longrightarrow} \Sigma X\right] } \\
& +\left[\Sigma X \stackrel{\left(\begin{array}{l}
0 \\
1
\end{array}\right)}{\longrightarrow} C^{f} \oplus \Sigma X \stackrel{(1,0)}{\longrightarrow} C^{f} \stackrel{0}{\longrightarrow} \Sigma X\right] .
\end{aligned}
$$

Combining (2.4.9), (2.4.10), and (2.4.11), and using (R9),

$$
\begin{aligned}
{\left[\Delta_{\mathrm{tr}}\right]+[\Delta]=} & {\left[\Gamma_{X}\right]-\left[\Sigma X \stackrel{\left(\begin{array}{l}
0 \\
1
\end{array}\right)}{\longrightarrow} C^{f} \oplus \Sigma X \stackrel{(1,0)}{\longrightarrow} C^{f} \stackrel{0}{\longrightarrow} \Sigma X\right] } \\
& +\left[C^{f} \stackrel{\left(\begin{array}{l}
1 \\
0
\end{array}\right)}{\longrightarrow} C^{f} \oplus \Sigma X \stackrel{(0,1)}{\longrightarrow} \Sigma X \stackrel{0}{\longrightarrow} \Sigma C^{f}\right] \\
= & {\left[\Gamma_{X}\right]+\left\langle\left[C^{f}\right],[\Sigma X]\right\rangle . }
\end{aligned}
$$

Theorem 2.4.12. The inclusion $j$ fits into a unique strong deformation retraction,

$$
{ }^{\alpha} \bigcap_{\mathcal{D}_{*}}(\bullet \mathscr{T}) \underset{j}{\stackrel{p}{\rightleftarrows}} \mathcal{D}_{*}(\mathscr{A}),
$$

where $\alpha: j p \Rightarrow 1$ satisfies the following equations, $\bullet=b, d, v$. Given an arbitrary object $X$ in $\mathscr{T}$ and a connective object $Y$ in $\mathscr{T}$,

$$
\begin{aligned}
0 & =\left[\Gamma_{X}\right]+\alpha([\Sigma X])^{[X]}+\alpha([X]), \\
\alpha([Y]) & =\left[\Delta_{Y}\right]+\alpha\left(\left[Y_{\leq-1}\right]\right) .
\end{aligned}
$$

Proof: We inductively define $\alpha$ on generators $[X]$. Let $X$ be a nontrivial object in $\mathscr{T}$. Since the $t$-structure is non-degenerate, $H^{*} X \neq 0$. Let $n \in \mathbb{Z}$ and $m \in \mathbb{Z}$ be the minimum and maximum integers such that 
$H^{n} X \neq 0 \neq H^{m} X$, respectively, i.e. $[n, m]$ is the smallest interval where the homology of $X$ is concentrated. We now define $p[X]$ by induction on $(m-n,|n|) \in \mathbb{N}^{2}$ with respect to the lexicographic order. If $m-n=0$ and $|n|=0$ then $X=A$ in $\mathscr{A}$, and we must define

$$
\alpha([A])=0
$$

so that $\alpha j=0$ holds. There are two kinds of induction steps: $(x, y) \rightarrow$ $(x, y+1)$ and $(x, y) \forall y \in \mathbb{N} \rightarrow(x+1,0)$. In the first case we define $\alpha([X])$ so that $(2.4 .13)$ is satisfied,

$$
\alpha([X])= \begin{cases}-\left[\Gamma_{\Sigma^{-1} X}\right]^{-\left[\Sigma^{-1} X\right]}-\alpha\left(\left[\Sigma^{-1} X\right]\right)^{-\left[\Sigma^{-1} X\right]}, & \text { if } n>0 \\ -\alpha([\Sigma X])^{[X]}-\left[\Gamma_{X}\right], & \text { if } n<0\end{cases}
$$

In the second case, $X=Y$ is connective, and we define $\alpha([Y])$ by (2.4.14).

We will now follow Lemmas 1.4.8 and 1.4.10. We first check that $f=1_{\mathcal{D}_{*}(\mathscr{T})}+\alpha$ factors through $j$, which is essentially an inclusion by Lemma 2.4.1. It is enough to prove that $f$ applied to any of the generators in Proposition 2.4.2 lies in the image of $j$. Given $A$ in $\mathscr{A}$, a short exact sequence $A \longmapsto B \rightarrow C$ in $\mathscr{A}$, and two objects $X$ and $Y$ in $\mathscr{T}$, with $Y$ connective,

$$
\begin{aligned}
& f[A]=[A]+\partial \alpha[A]=[A]+\partial(0)=[A], \\
& f[A \longleftrightarrow B \longrightarrow C]=[A \succ B \longrightarrow C]+\alpha \partial[A \longleftrightarrow B \longrightarrow C] \\
& =[A \succ B \longrightarrow C]+\alpha(-[B]+[C]+[A]) \\
& =[A \longleftrightarrow B \longrightarrow C]-\alpha([B])^{-[B]+[C]+[A]}+\alpha([C])^{[A]}+\alpha[A] \\
& \text { (2.4.15) } \\
& =[A \longmapsto B \longrightarrow C], \\
& f\left[\Gamma_{X}\right]=\left[\Gamma_{X}\right]+\alpha \partial\left[\Gamma_{X}\right] \\
& =\left[\Gamma_{X}\right]+\alpha([\Sigma X]+[X]) \\
& =\left[\Gamma_{X}\right]+\alpha([\Sigma X])^{[X]}+\alpha[X] \\
& \text { (2.4.13) }=0 \text {, } \\
& f\left[\Delta_{Y}\right]=\left[\Delta_{Y}\right]+\alpha \partial\left[\Delta_{Y}\right] \\
& =\left[\Delta_{Y}\right]+\alpha\left(-[Y]+\left[H^{0} Y\right]+\left[Y_{\leq-1}\right]\right) \\
& =\left[\Delta_{Y}\right]-\alpha([Y])^{\partial\left[\Delta_{Y}\right]}+\alpha\left(\left[H^{0} Y\right]\right)^{\left[Y_{\leq-1}\right]}+\alpha\left(\left[Y_{\leq-1}\right]\right) \\
& \text { (2.4.15) } \\
& =-\alpha([Y])+\left[\Delta_{Y}\right]+\alpha\left(\left[Y_{\leq-1}\right]\right) \\
& =0 \text {. }
\end{aligned}
$$

The first two equations also prove that $f j=j$. 
The homotopy $\alpha$ has been defined so that $\alpha[A]=0$ for any object $A$ in $\mathscr{A}$. Hence, it is only left to prove that

$$
\alpha([X]+\partial \alpha[X])=0 .
$$

In order to check this equation, we follow the same induction pattern as for the definition of $\alpha[X]$. If $X$ is in $\mathscr{A}$ then (2.4.17) follows immediately from (2.4.15). Equation (2.4.17) for $[X]$ is equivalent to equation (2.4.17) for $[\Sigma X]$. Indeed, applying $\partial$ to (2.4.13) we obtain

$$
0=[\Sigma X]+\partial \alpha[\Sigma X]+[X]+\partial \alpha[X] .
$$

Hence

$$
0=\alpha(0)=\alpha([\Sigma X]+\partial \alpha[\Sigma X])^{[X]+\partial \alpha[X] .}+\alpha([X]+\partial \alpha[X]) .
$$

Finally, if $Y$ is connective then (2.4.17) for $[Y]$ is equivalent to (2.4.17) for $\left[Y_{\leq-1}\right]$. Actually, applying $\partial$ to (2.4.14) we derive

$$
\partial \alpha[Y]=-[Y]+\left[H^{0} Y\right]+\left[Y_{\leq-1}\right]+\partial \alpha\left[Y_{\leq-1}\right] .
$$

Therefore,

$$
\begin{aligned}
\alpha([Y]+\partial \alpha(Y)) & =\alpha\left(\left[H^{0} Y\right]+\left[Y_{\leq-1}\right]+\partial \alpha\left[Y_{\leq-1}\right]\right) \\
& =\alpha\left(\left[H^{0} Y\right]\right)^{\left[Y_{\leq-1}\right]+\partial \alpha\left[Y_{\leq-1}\right]}+\alpha\left(\left[Y_{\leq-1}\right]+\partial \alpha\left[Y_{\leq-1}\right]\right) \\
& =\alpha\left(\left[Y_{\leq-1}\right]+\partial \alpha\left[Y_{\leq-1}\right]\right) .
\end{aligned}
$$

Corollary 2.4.18. The comparison maps in Theorem 1.7.5 induce isomorphisms

$$
K_{i}(\mathscr{A}) \cong K_{i}\left({ }^{d} \mathscr{T}\right) \cong K_{i}\left({ }^{b} \mathscr{T}\right) \cong K_{i}\left({ }^{v} \mathscr{T}\right), \quad i=0,1 .
$$

Definition 2.4.19. For $\bullet=b, d, v$, we define $\mathcal{D}_{*}^{t}(\bullet \mathscr{T})$ as the quotient of $\mathcal{D}_{*}(\mathscr{T})$ by the following relations:

(R11) $\left[\Delta_{Y}\right]=0$ for any connective object $Y$.

(R12) $\left[\Gamma_{X}\right]=0$ for any object $X$.

Proposition 2.4.20. The composite of the morphism $j$ with the natural projection is an isomorphism, $=b, d, v$,

$$
\bar{\jmath}: \mathcal{D}_{*}(\mathscr{A}) \stackrel{\cong}{\longrightarrow} \mathcal{D}_{*}^{t}(\bullet \mathscr{T}) .
$$

Proof: We use the notation in Theorem 2.4.12. We have seen in its proof that the morphism $p$ satisfies $p\left[\Delta_{Y}\right]=0=p\left[\Gamma_{Y}\right]$, hence it factors through $\mathcal{D}_{*}(\bullet \mathscr{T})$. Denote $\bar{p}$ the (unique) factorization. We are going to check that

$$
\mathcal{D}_{*}^{t}(\bullet \mathscr{T}) \underset{\bar{\jmath}}{\stackrel{\bar{p}}{\rightleftarrows}} \mathcal{D}_{*}(\mathscr{A})
$$


are mutually inverse isomorphisms. The equation $\bar{p} \bar{\jmath}=1$ follows from $p j=1$. In order to check that $\bar{\jmath}=1$ it is enough to notice that the image of $\alpha$ vanishes in $\mathcal{D}_{1}^{t}(\bullet \mathscr{T})$. This follows easily from (2.4.13) and (2.4.14) by the induction procedure applied twice in the previous proof.

Remark 2.4.21. This proposition admits a direct proof. Indeed, the equations in the proof of Theorem 2.4.12 yield an inductive formula for $p$. Using this formula one can easily check that $\bar{p} \bar{\jmath}=1$. The proof of $\bar{p} \bar{p}=1$ is straightforward, but rather tedious. A previous version of this paper contained a sketch. We invite the reader interested in practicing with the algebra of stable quadratic modules to reconstruct it as an exercise.

The advantage of a direct proof is that we would obtain Lemma 2.4.1 as an immediate corollary, avoiding invoking Neeman's result, whose proof is long and complicated.

2.5. The $K$-theory of some unusual triangulated categories. Let $R$ be a commutative local ring with maximal ideal $(\varepsilon) \neq 0$ such that $\varepsilon^{2}=0$ and with residue field $k=R /(\varepsilon)$ of characteristic 2. This ring is quasi-Frobenius. Notice that either $\varepsilon=2$ or $R=k[\varepsilon] / \varepsilon^{2}$. Recall from [MSS] that the category $\mathcal{F}(R)$ of finitely generated free $R$-modules admits a unique triangulated structure with identity suspension functor $\Sigma=1_{\mathcal{F}(R)}$ such that the following triangle is distinguished,

$$
\Delta_{\varepsilon}: R \stackrel{\varepsilon}{\longrightarrow} R \stackrel{\varepsilon}{\longrightarrow} R \stackrel{\varepsilon}{\longrightarrow} R \text {. }
$$

This triangulated category does not admit models if $\varepsilon=2$. Otherwise it is the compact derived category of a certain differential graded algebra, in particular it can be described as the homotopy category of a Waldhausen category.

Theorem 2.5.1. Neeman's and Breuning's K-theories of the triangulated category $\mathcal{F}(R)$ satisfy:

$$
\begin{aligned}
& K_{0}\left({ }^{b} \mathcal{F}(R)\right) \cong K_{0}\left({ }^{d} \mathcal{F}(R)\right) \cong K_{0}\left({ }^{v} \mathcal{F}(R)\right) \cong 0, \\
& K_{1}\left({ }^{b} \mathcal{F}(R)\right) \cong K_{1}\left({ }^{d} \mathcal{F}(R)\right) \cong 0 .
\end{aligned}
$$

Moreover, there is a surjective homomorphism $K_{1}\left({ }^{v} \mathcal{F}(R)\right) \rightarrow k^{\times} /\left(k^{\times}\right)^{2}$.

Notice that $k^{\times} /\left(k^{\times}\right)^{2} \neq 0$ as long as $k$ is non-perfect, thus we obtain examples of triangulated categories $\mathscr{T}$ such that $K_{1}\left({ }^{b} \mathscr{T}\right)$ and $K_{1}\left({ }^{d} \mathscr{T}\right)$ are not isomorphic to $K_{1}\left({ }^{v} \mathscr{T}\right)$.

An acyclic 3 -periodic complex in $\mathcal{F}(R)$,

$$
\Delta: X_{0} \stackrel{d_{2}}{\longrightarrow} X_{2} \stackrel{d_{1}}{\longrightarrow} X_{1} \stackrel{d_{0}}{\longrightarrow} X_{0},
$$


fits into a natural short exact sequence of complexes,

$$
\varepsilon \cdot \Delta \longmapsto \Delta \stackrel{\varepsilon}{\longrightarrow} \varepsilon \cdot \Delta,
$$

which induces isomorphisms in homology,

$$
\sigma_{n}^{\Delta}: H_{n+1}(\varepsilon \cdot \Delta) \cong H_{n}(\varepsilon \cdot \Delta), \quad n \in \mathbb{Z} / 3 .
$$

A distinguished triangle in $\mathcal{F}(R)$ is the same as an acyclic 3-periodic chain complex $\Delta$ such that the automorphism

$$
\rho_{n}^{\Delta}=\sigma_{n}^{\Delta} \sigma_{n+1}^{\Delta} \sigma_{n+2}^{\Delta}: H_{n}(\varepsilon \cdot \Delta) \cong H_{n}(\varepsilon \cdot \Delta)
$$

is the identity for some, and hence all, $n \in \mathbb{Z} / 3$, see [MSS, Remark 7].

Definition 2.5.2. We define the determinant of an acyclic 3-periodic complex $\Delta$ in $\mathcal{F}(R)$ as $\operatorname{det}(\Delta)=\operatorname{det}\left(\rho_{n}^{\Delta}\right) \in k^{\times}$, which is independent of $n \in \mathbb{Z} / 3$.

The determinant is clearly invariant under shifts of the complex $\Delta$ and isomorphisms. Notice that the determinant of a distinguished triangle is $1 \in k^{\times}$.

Lemma 2.5.3. Given a short exact sequence of acyclic 3-periodic complexes $\Delta^{\prime} \longmapsto \Delta \rightarrow \Delta^{\prime \prime}$ in $\mathcal{F}(R)$ we have $\operatorname{det}(\Delta)=\operatorname{det}\left(\Delta^{\prime}\right) \operatorname{det}\left(\Delta^{\prime \prime}\right)$ $\bmod \left(k^{\times}\right)^{2}$.

Proof: The short exact sequence in the statement splits in each degree, so we have a short exact sequence $\varepsilon \cdot \Delta^{\prime} \longmapsto \varepsilon \cdot \Delta \rightarrow \varepsilon \cdot \Delta^{\prime \prime}$ which induces a long exact sequence in homology,

$\cdots \longrightarrow H_{n}\left(\varepsilon \cdot \Delta^{\prime}\right) \longrightarrow H_{n}(\varepsilon \cdot \Delta) \longrightarrow H_{n}\left(\varepsilon \cdot \Delta^{\prime \prime}\right) \longrightarrow H_{n-1}\left(\varepsilon \cdot \Delta^{\prime}\right) \longrightarrow \cdots$.

Moreover, the following $3 \times 3$ diagram of short exact sequences of complexes

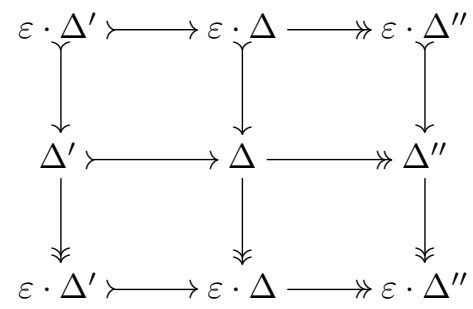


shows that the following diagram is commutative, since we are in characteristic 2,

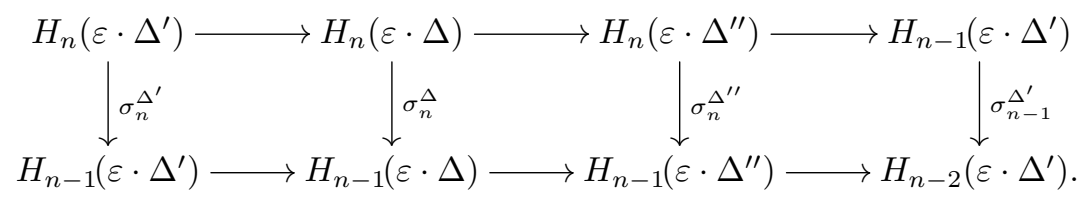

Therefore we have an automorphism of a 9-periodic long exact sequence, $n \in \mathbb{Z} / 3$,

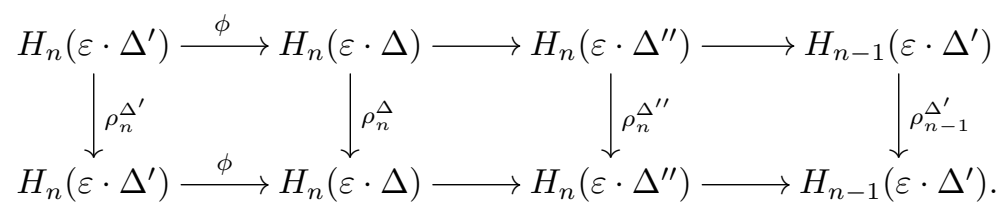

Using the multiplicative property of determinants with respect to automorphisms of short exact sequences, if $\rho^{\prime}: \operatorname{Ker} \phi \cong \operatorname{Ker} \phi$ is the automorphism induced by $\rho_{n}^{\Delta^{\prime}}$ we get

$$
\begin{aligned}
\operatorname{det}\left(\rho^{\prime}\right)^{2}= & \operatorname{det}\left(\rho_{n}^{\Delta^{\prime}}\right) \operatorname{det}\left(\rho_{n}^{\Delta}\right)^{-1} \operatorname{det}\left(\rho_{n}^{\Delta^{\prime \prime}}\right) \\
& \operatorname{det}\left(\rho_{n-1}^{\Delta^{\prime}}\right)^{-1} \operatorname{det}\left(\rho_{n-1}^{\Delta}\right) \operatorname{det}\left(\rho_{n-1}^{\Delta^{\prime \prime}}\right)^{-1} \\
& \operatorname{det}\left(\rho_{n-2}^{\Delta^{\prime}}\right) \operatorname{det}\left(\rho_{n-2}^{\Delta}\right)^{-1} \operatorname{det}\left(\rho_{n-2}^{\Delta^{\prime \prime}}\right) .
\end{aligned}
$$

Since these determinants are independent of $n \in \mathbb{Z} / 3$ we deduce, as desired, that

$$
\operatorname{det}\left(\rho^{\prime}\right)^{2} \operatorname{det}\left(\rho_{n}^{\Delta}\right)=\operatorname{det}\left(\rho_{n}^{\Delta^{\prime}}\right) \operatorname{det}\left(\rho_{n}^{\Delta^{\prime \prime}}\right)
$$

Lemma 2.5.4. A virtual triangle in $\mathcal{F}(R)$ is the same as an acyclic 3-periodic complex $\Delta$. Moreover, $\Delta$ is the direct sum of a contractible triangle and

$$
R^{d} \stackrel{\varepsilon}{\longrightarrow} R^{d} \stackrel{\varepsilon}{\longrightarrow} R^{d} \stackrel{\varepsilon \cdot \bar{\rho}}{\longrightarrow} R^{d},
$$

where $d=\operatorname{dim}_{k} H_{n}(\varepsilon \cdot \Delta)$ and $\bar{\rho}$ is any automorphism of $R^{d}$ with $\bar{\rho} \otimes_{R} k=$ $\rho_{n}^{\Delta}$ for some basis of $H_{n}(\varepsilon \cdot \Delta), n \in \mathbb{Z} / 3$.

Proof: It is clear that a virtual triangle is acyclic. Consider an acyclic 3-periodic complex in $\mathcal{F}(R)$,

$$
\Delta: X_{0} \stackrel{d_{2}}{\longrightarrow} X_{2} \stackrel{d_{1}}{\longrightarrow} X_{1} \stackrel{d_{0}}{\longrightarrow} X_{0} .
$$


Let $X_{n}^{\prime} \subset \operatorname{Ker} d_{n} \subset X_{n}$ be an injective envelope of $\varepsilon \cdot \operatorname{Ker} d_{n}$. Since $\Delta$ is acyclic, we can factor this inclusion as

$$
X_{n}^{\prime} \longrightarrow X_{n+1} \stackrel{d_{n}}{\longrightarrow} X_{n} .
$$

This allows us to split $\Delta=\Delta^{\prime} \oplus \Delta^{\prime \prime}$ as the direct sum of a contractible factor $\Delta^{\prime}$ and a second factor $\Delta^{\prime \prime}$,

$$
\begin{aligned}
& \Delta^{\prime}: X_{0}^{\prime} \oplus X_{2}^{\prime} \stackrel{\left(\begin{array}{ll}
0 & 1 \\
0 & 0
\end{array}\right)}{\longrightarrow} X_{2}^{\prime} \oplus X_{1}^{\prime} \stackrel{\left(\begin{array}{ll}
0 & 1 \\
0 & 0
\end{array}\right)}{\longrightarrow} X_{1}^{\prime} \oplus X_{0}^{\prime} \stackrel{\left(\begin{array}{ll}
(0 & 1 \\
0 & 0
\end{array}\right)}{\longrightarrow} X_{0}^{\prime} \oplus X_{2}^{\prime}, \\
& \Delta^{\prime \prime}: X_{0}^{\prime \prime} \stackrel{d_{2}^{\prime \prime}}{\longrightarrow} X_{2}^{\prime \prime} \stackrel{d_{1}^{\prime \prime}}{\longrightarrow} X_{1}^{\prime \prime} \stackrel{d_{0}^{\prime \prime}}{\longrightarrow} X_{0}^{\prime \prime},
\end{aligned}
$$

with $\operatorname{Im} d_{n}^{\prime \prime}=\operatorname{Ker} d_{n-1}^{\prime \prime} \subset \varepsilon \cdot X_{n-1}$, so $d_{n}^{\prime \prime}=\varepsilon \cdot \bar{d}_{n}$ for some $\bar{d}_{n}: X_{n+1} \rightarrow$ $X_{n}$. One can easily check that $\sigma_{n}^{\Delta}=\bar{d}_{n} \otimes_{R} k$, therefore $\bar{d}_{n} \otimes_{R} k$, and hence $\bar{d}_{n}$, is an isomorphism. Now the following isomorphism of 3 -periodic complexes proves the lemma

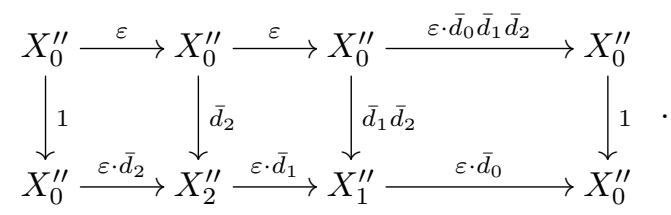

Lemma 2.5.5. Given a virtual octahedron in $\mathcal{F}(R)$

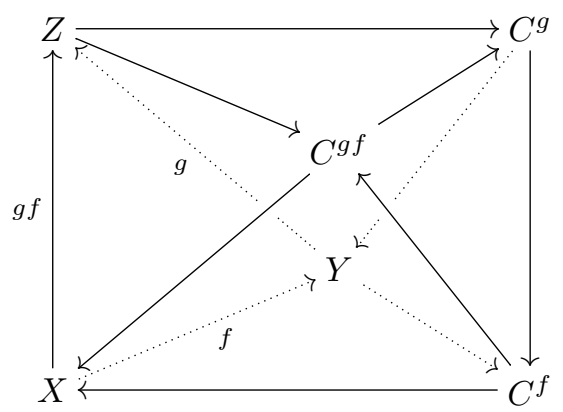

formed by virtual triangles $\Delta_{f}, \Delta_{g}, \Delta_{g f}, \widetilde{\Delta}$, the following formula holds,

$$
\operatorname{det}\left(\Delta_{g}\right) \operatorname{det}\left(\Delta_{f}\right)=\operatorname{det}\left(\Delta_{g f}\right) \operatorname{det}(\widetilde{\Delta}) \bmod \left(k^{\times}\right)^{2} .
$$


Proof: The octahedron contains morphisms of complexes,

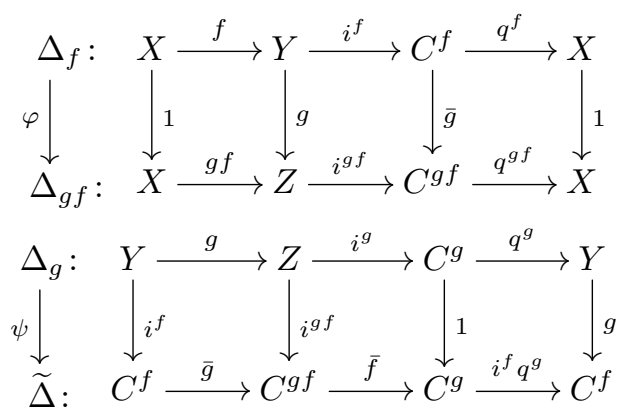

The mapping cones of these morphisms fit in the middle of well known short exact sequences of complexes involving the target and a translation of the source, hence by Lemma 2.5.3,

$$
\begin{aligned}
& \operatorname{det}(\operatorname{Cone}(\varphi))=\operatorname{det}\left(\Delta_{g f}\right) \operatorname{det}\left(\Delta_{f}\right) \bmod \left(k^{\times}\right)^{2}, \\
& \operatorname{det}(\operatorname{Cone}(\psi))=\operatorname{det}(\widetilde{\Delta}) \operatorname{det}\left(\Delta_{g}\right) \quad \bmod \left(k^{\times}\right)^{2} .
\end{aligned}
$$

In this case they also fit into the following short exact sequences,

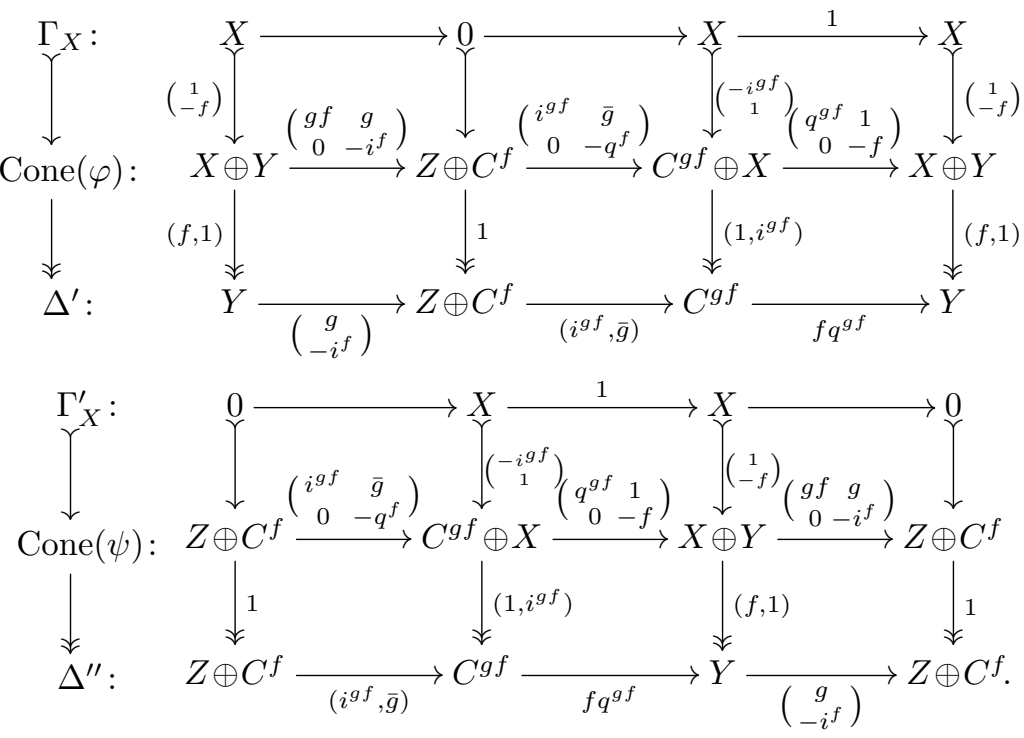


Moreover, $\Delta^{\prime \prime}$ is the translation of $\Delta^{\prime}$. Therefore, using again Lemma 2.5.3,

$$
\begin{array}{ll}
\operatorname{det}(\operatorname{Cone}(\varphi))=\operatorname{det}\left(\Gamma_{X}\right) \operatorname{det}\left(\Delta^{\prime}\right)=\operatorname{det}\left(\Delta^{\prime}\right) & \bmod \left(k^{\times}\right)^{2}, \\
\operatorname{det}(\operatorname{Cone}(\psi))=\operatorname{det}\left(\Gamma_{X}^{\prime}\right) \operatorname{det}\left(\Delta^{\prime \prime}\right)=\operatorname{det}\left(\Delta^{\prime}\right) & \bmod \left(k^{\times}\right)^{2},
\end{array}
$$

so we are done.

Proof of Theorem 2.5.1: In this proof, $=b, d$. We can suppose that the objects of $\mathcal{F}(R)$ are simply $R^{n}, n \geq 0$, hence Proposition 1.5.7 applies. Generators (G2) vanish in $\mathcal{D}_{*}^{+}(\bullet \mathcal{F}(R))$. Indeed, given an isomorphism $h: R^{n} \cong R^{n}$, the distinguished triangle isomorphism

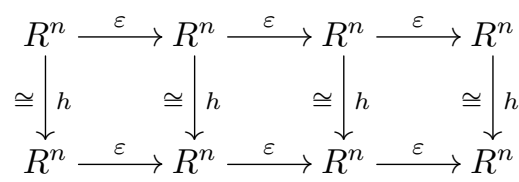

together with (R7) and (R10) yield

$[h]+[h]^{n[R]}=-n\left[\Delta_{\varepsilon}\right]+[h]+n\left[\Delta_{\varepsilon}\right]=-n\left[\Delta_{\varepsilon}\right]+n\left[\Delta_{\varepsilon}\right]+[h]^{\partial\left(n\left[\Delta_{\varepsilon}\right]\right)}=[h]^{n[R]}$, therefore $[h]=0$. In particular, the bracket vanishes in $\mathcal{D}_{*}^{+}(\bullet \mathcal{F}(R))$ by $\left(\mathrm{R} 9^{\prime}\right)$. Moreover, $2\left[\Delta_{\varepsilon}\right]=\left[\Gamma_{X}\right]$ by Lemma 2.4.8. Furthermore, (R7) shows that two isomorphic distinguished triangles represent the same (G3) generator. Any exact triangle in $\mathcal{F}(R)$ is a direct sum of a contractible triangle and copies of $\Delta_{\varepsilon}$, hence $\mathcal{D}_{*}^{+}(\bullet \mathcal{F}(R))$ is generated by $[R]$ and $\left[\Delta_{\epsilon}\right]$, see Remark 1.5.6. Let $\alpha: f \Rightarrow 0$ be the homotopy to the trivial endomorphism of $\mathcal{D}_{*}^{+}(\bullet \mathcal{F}(R))$ defined by Lemma 1.4.8 and $\alpha([R])=\left[\Delta_{\varepsilon}\right]$. We have

$$
\begin{gathered}
f([R])=\partial \alpha([R])=\partial\left(\left[\Delta_{\varepsilon}\right]\right)=-[R]+[R]+[R]=[R], \\
f\left(\left[\Delta_{\varepsilon}\right]\right)=\alpha \partial\left(\left[\Delta_{\varepsilon}\right]\right)=\alpha(-[R]+[R]+[R])=\alpha([R])=\left[\Delta_{\varepsilon}\right] .
\end{gathered}
$$

Thus $f$ is the identity, i.e. $\mathcal{D}_{*}^{+}(\bullet \mathcal{F}(R))$ is contractible. In particular $K_{i}(\bullet \mathcal{F}(R)) \cong \pi_{i} \mathcal{D}_{*}^{+}(\bullet \mathcal{F}(R))=0, i=0,1$. Recall also that $K_{0}\left({ }^{v} \mathcal{F}(R)\right)=$ $K_{0}(\bullet \mathcal{F}(R))$.

Let us regard the abelian group $k^{\times} /\left(k^{\times}\right)^{2}$ as a stable quadratic module concentrated in degree 1. By Proposition 1.5.3 and Lemma 2.5.5 the determinant of virtual triangles defines a morphism $p: \mathcal{D}_{*}\left({ }^{v} \mathcal{F}(R)\right) \rightarrow$ $k^{\times} /\left(k^{\times}\right)^{2}$ with $p[\Delta]=\operatorname{det}(\Delta)$ for any virtual triangle $\Delta$. The induced morphism $\pi_{1}(p): K_{1}\left({ }^{v} \mathcal{F}(R)\right) \rightarrow k^{\times} /\left(k^{\times}\right)^{2}$ is surjective since

$$
p[R \underset{\varepsilon}{\stackrel{\varepsilon}{\longrightarrow}} R \underset{\varepsilon}{\stackrel{\varepsilon}{\longrightarrow}} R \underset{\varepsilon \cdot \lambda}{\stackrel{\varepsilon}{\longrightarrow}} R]=\operatorname{det}\left(\Delta_{\varepsilon}\right)^{-1} \operatorname{det}(R \stackrel{\varepsilon}{\longrightarrow} R \stackrel{\varepsilon}{\longrightarrow} R \stackrel{\varepsilon \cdot \lambda}{\longrightarrow} R)=\lambda .
$$


2.6. A counterexample to two conjectures by Maltsiniotis. Based on the following example, which goes back to Deligne, Vaknin, Ferrand, and Breuning [Vak1, Fer, Bre2], we disprove two conjectures due to Maltsiniotis.

Let $\mathscr{E}=\operatorname{proj}(R)$ be the category of finitely generated free modules over the ring of dual numbers $R=k[\varepsilon] / \varepsilon^{2}$ over a field $k$. We regard $D^{b}(\mathscr{E})$ as a strongly triangulated category with the structure indicated in [Mal1]. It is well known that $K_{1}(\mathscr{E}) \cong K_{1}(R) \cong R^{\times}$is the group of units. There is an isomorphism, $k \times k^{\times} \cong R^{\times}:(x, u) \mapsto u(1+x \varepsilon)$. Given $x \in k$, the element $1+x \varepsilon \in R^{\times}$corresponds to

$$
[1+x \varepsilon: R \stackrel{\sim}{\longrightarrow} R] \in K_{1}(\mathscr{E}) .
$$

This element is in the kernel of $K_{1}(\mathscr{E}) \rightarrow K_{1}\left({ }^{s} D^{b}(\mathscr{E})\right)$ since we have the following automorphism of distinguished triangles, see (R7),

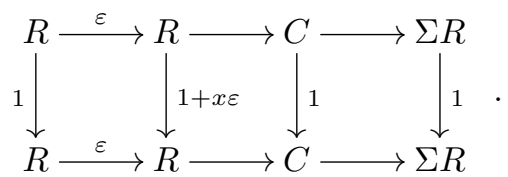

Indeed, $C$ is the complex $\cdots \rightarrow 0 \rightarrow R \stackrel{\varepsilon}{\rightarrow} R \rightarrow 0 \rightarrow \cdots$, and the square in the middle commutes in the derived category since we have a homotopy defined by the homomorphism $R \rightarrow R: 1 \mapsto x$. Maltsiniotis conjectured in [Mal1] that $K_{n}(\mathscr{E}) \rightarrow K_{n}\left({ }^{s} D^{b}(\mathscr{E})\right)$ would be an isomorphism for all $n \geq 0$.

The same example shows that, if we only regard $D^{b}(\mathscr{E})$ as a triangulated category, then the comparison homomorphisms,

$$
\begin{aligned}
& K_{1}(\mathscr{E}) \longrightarrow K_{1}\left({ }^{b} D^{b}(\mathscr{E})\right), \\
& K_{1}(\mathscr{E}) \longrightarrow K_{1}\left({ }^{d} D^{b}(\mathscr{E})\right), \\
& K_{1}(\mathscr{E}) \longrightarrow K_{1}\left({ }^{v} D^{b}(\mathscr{E})\right),
\end{aligned}
$$

are not isomorphisms.

Moreover, if $\mathbb{D}_{\mathscr{E}}$ is the triangulated derivator associated to $\mathscr{E}[\mathbf{K e l}]$, the comparison homomorphism $K_{1}\left(\mathbb{D}_{\mathscr{E}}\right) \rightarrow K_{1}\left({ }^{s} \mathbb{D}_{\mathscr{E}}(*)\right)$ in $[$ Mal1] is not an isomorphism either, because the composite

$$
K_{1}(\mathscr{E}) \stackrel{\cong}{\longrightarrow} K_{1}\left(\mathbb{D}_{\mathscr{E}}\right) \longrightarrow K_{1}\left({ }^{s} \mathbb{D}_{\mathscr{E}}(*)\right) \cong K_{1}\left({ }^{s} D^{b}(\mathscr{E})\right)
$$

is the previous comparison homomorphism between Quillen's $K$-theory and Maltsiniotis $K$-theory of a strongly triangulated category, which is not injective. The first arrow is the natural comparison homomorphism in [Mal2], which is an isomorphism by [Mur, Theorem 1]. Maltsiniotis 
also conjectured that $K_{n}\left(\mathbb{D}_{\mathscr{E}}\right) \rightarrow K_{n}\left(s \mathbb{D}_{\mathscr{E}}(*)\right)$ would be an isomorphism for all $n \geq 0$, see [Mal1].

We can actually compute Neeman's $K_{1}\left({ }^{d} D^{b}(\mathscr{E})\right)$ and Breuning's $K_{1}\left({ }^{b} D^{b}(\mathscr{E})\right)$. This improves and generalizes some computations in [Bre2].

Proposition 2.6.1. For $\bullet=b, d$, the stable quadratic module $\mathcal{D}_{*}\left(\bullet D^{b}(\mathscr{E})\right)$ is weakly equivalent to

$$
\mathbb{Z} \otimes \mathbb{Z} \stackrel{\langle\cdot, \cdot\rangle}{\longrightarrow} k^{\times} \stackrel{\partial}{\longrightarrow} \mathbb{Z}, \quad\langle 1,1\rangle=-1, \quad \partial=0 .
$$

Moreover, the comparison homomorphism,

$$
k \times k^{\times} \cong K_{1}\left(k[\varepsilon] / \varepsilon^{2}\right) \longrightarrow K_{1}\left(D^{b}(\mathscr{E})\right) \cong k^{\times},
$$

is the natural projection onto the second factor.

Proof: Theorem 2.4.12 and Remark 1.5.11 show that $\mathcal{D}_{*}\left(\bullet D^{b}(k)\right)$ is weakly equivalent to the stable quadratic module in the statement. We have already seen that the subgroup $k \subset k \times k^{\times}$is in the kernel of the comparison homomorphism, which is known to be surjective, see Subsection 1.7. Therefore, it induces an epimorphism, $k^{\times} \rightarrow K_{1}\left({ }^{d} D^{b}\left(k[\varepsilon] / \varepsilon^{2}\right)\right)$. This epimorphism is also injective since the following composite is the identity,

$$
k^{\times} \longrightarrow K_{1}\left({ }^{d} D^{b}\left(k[\varepsilon] / \varepsilon^{2}\right)\right) \longrightarrow K_{1}\left({ }^{d} D^{b}(k)\right) \cong k^{\times} .
$$

Here the second arrow is induced by the change of coefficients along the $k$-algebra morphism $k[\varepsilon] / \varepsilon^{2} \rightarrow k: \varepsilon \mapsto 0$. In particular, the map $\mathcal{D}_{*}\left(\bullet D^{b}(\mathscr{E})\right) \rightarrow \mathcal{D}_{*}\left(\bullet D^{b}(k)\right)$ induced by the previous change of coefficients is an isomorphism in $\pi_{1}$. It is also an isomorphism in $\pi_{0}$, since $K_{0}(R) \cong K_{0}(k) \cong \mathbb{Z}$ generated by the free module of rank 1 , compare again Subsection 1.7. Hence we are done.

\section{Strict Picard groupoids}

In this section we review strictification results for Picard groupoids and related categorical structures. This theory is essential for the proof of our main results on determinant functors.

3.1. Categorical groups. A monoidal groupoid $(\mathscr{G}, \otimes, I)$ with unit object $I$ is a categorical group if for each object $x$ of $\mathscr{G}$ there is an object $x^{*}$ and a map $j_{x}: x^{*} \otimes x \cong I$. Equivalently, there is a contravariant functor $*$ on $\mathscr{G}$ such that the endofunctors $\_x$ and $x \otimes_{-}$are equivalences of categories with inverses _ $\otimes x^{*}$ and $x^{*} \otimes_{-}$, respectively [Lap]. 
A categorical group is braided or symmetric if the underlying monoidal category is. Recall that a braiding is a natural isomorphism,

$$
\operatorname{comm}_{x, y}: x \otimes y \longrightarrow y \otimes x,
$$

satisfying certain coherence laws $[\mathbf{J S}]$, and is a symmetry if $\operatorname{comm}_{y, x} \circ$ $\operatorname{comm}_{x, y}=1_{x \otimes y}$ is the identity. A Picard groupoid is just a symmetric categorical group.

A tensor functor between categorical groups is a functor $F: \mathscr{G} \rightarrow \mathscr{H}$ together with comparison maps for tensor units and multiplication,

$$
\text { unit: } I_{\mathscr{H}} \longrightarrow F\left(I_{\mathscr{G}}\right), \quad \operatorname{mult}_{x, y}: F(x) \otimes F(y) \longrightarrow F(x \otimes y),
$$

which are natural and compatible with the associativity and unit isomorphisms [Eps]. A tensor functor between braided (or symmetric) categorical groups is symmetric if it is also compatible with the braiding isomorphisms. A tensor natural transformation $\alpha: F \Rightarrow G$ is one which commutes with the comparison maps for multiplication. (Braided, symmetric) categorical groups, (symmetric) tensor functors and tensor natural transformations form 2-categories, more specifically, categories enriched in groupoids. Notice that the obvious forgetful 2-functors

symmetric cat. groups $\longrightarrow$ braided cat. groups $\longrightarrow$ cat. groups

are faithful in dimension 1 and fully faithful in dimension 2, i.e. injective on tensor functors and bijective on tensor natural transformations.

The homotopy groups of a (braided, symmetric) categorical group $\mathscr{G}$ are,

$$
\begin{aligned}
& \pi_{0}(\mathscr{G})=\text { isomorphism classes of objects, with }+ \text { induced by } \otimes, \\
& \pi_{1}(\mathscr{G})=\operatorname{Aut} \mathscr{G}(I) .
\end{aligned}
$$

Homotopy groups detect equivalences. The group $\pi_{0}(\mathscr{G})$ acts on $\pi_{1}(\mathscr{G})$ by

$$
x^{*} \otimes x \otimes(I \stackrel{f}{\longrightarrow} I)^{[x]}=x^{*} \otimes(I \stackrel{f}{\longrightarrow} I) \otimes x: x^{*} \otimes x \longrightarrow x^{*} \otimes x,
$$

and the action is trivial in the braided case. One can define the $k$-invariant in the braided case as the natural quadratic map

$$
\eta: \pi_{0}(\mathscr{G}) \longrightarrow \pi_{1}(\mathscr{G})
$$

such that $x \otimes x \otimes \eta([x])=\operatorname{comm}_{x, x}$, and $\mathscr{G}$ is symmetric if and only if the $k$-invariant factors through a homomorphism

$$
\eta: \pi_{0}(\mathscr{G}) \otimes \mathbb{Z} / 2 \longrightarrow \pi_{1}(\mathscr{G}) .
$$

A (braided, symmetric) categorical group is strict if the associativity and unit isomorphisms are identities and the isomorphisms $j_{x}$ can be 
chosen to be identities. Thus the underlying monoidal category is strict and the functors $\otimes_{-} \otimes$ and $x \otimes$ _ are isomorphisms of categories. If $\mathscr{G}$ and $\mathscr{H}$ are strict then $F: \mathscr{G} \rightarrow \mathscr{H}$ is a strict tensor functor if the comparison maps for multiplication are all identities.

Strict (braided, symmetric) categorical groups, strict (symmetric) tensor functors and tensor natural transformations again form a 2-category.

3.2. Crossed modules. Recall that a crossed module is a group homomorphism $\partial: C_{1} \rightarrow C_{0}$ together with a right action of $C_{0}$ on $C_{1}$ such that, for $c_{i}, c_{i}^{\prime} \in C_{i}$,

(1) $\partial\left(c_{1}{ }^{c_{0}}\right)=-c_{0}+\partial\left(c_{1}\right)+c_{0}$,

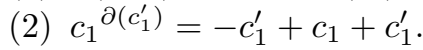

We denote the group laws additively, although the groups may be nonabelian. It follows that the image of $\partial$ is always a normal subgroup, and the kernel is always central. The homotopy groups of $C_{*}$ are defined as in Definition 1.4.5. The action of $C_{0}$ on $C_{1}$ induces an action of $\pi_{0}\left(C_{*}\right)$ on $\pi_{1}\left(C_{*}\right)$.

A reduced 2-crossed module, or simply reduced 2-module, is a crossed module together with a map,

$$
\langle\cdot, \cdot\rangle: C_{0} \times C_{0} \longrightarrow C_{1},
$$

which controls commutators. It must satisfy:

(3) $\partial\left\langle c_{0}, c_{0}^{\prime}\right\rangle=\left[c_{0}^{\prime}, c_{0}\right]$

(4) $c_{1}^{c_{0}}=c_{1}+\left\langle c_{0}, \partial\left(c_{1}\right)\right\rangle$,

(5) $\left\langle c_{0}, \partial\left(c_{1}\right)\right\rangle+\left\langle\partial\left(c_{1}\right), c_{0}\right\rangle=0$,

(6) $\left\langle c_{0}, c_{0}^{\prime}+c_{0}^{\prime \prime}\right\rangle=\left\langle c_{0}, c_{0}^{\prime}\right\rangle^{c_{0}^{\prime \prime}}+\left\langle c_{0}, c_{0}^{\prime \prime}\right\rangle$,

(7) $\left\langle c_{0}+c_{0}^{\prime}, c_{0}^{\prime \prime}\right\rangle=\left\langle c_{0}^{\prime}, c_{0}^{\prime \prime}\right\rangle+\left\langle c_{0}, c_{0}^{\prime \prime}\right\rangle c_{0}^{\prime}$.

The crossed module $\partial$ and the bracket $\langle\cdot, \cdot\rangle$ form a stable 2 -crossed module, or simply stable 2 -module, if (3), (4), (6), and

(8) $\left\langle c_{0}, c_{0}^{\prime}\right\rangle+\left\langle c_{0}^{\prime}, c_{0}\right\rangle=0$

are satisfied. In a reduced or stable 2-module the action of $C_{0}$ on $C_{1}$ is completely determined by the bracket $\langle\cdot, \cdot\rangle$, by (4), so (1) is redundant and (2) becomes

(9) $\left\langle\partial\left(c_{1}\right), \partial\left(c_{1}^{\prime}\right)\right\rangle=\left[c_{1}^{\prime}, c_{1}\right]$.

The $k$-invariant of a reduced 2-module $C_{*}$ is the natural quadratic map,

$$
\begin{aligned}
\eta: \pi_{0}\left(C_{*}\right) & \longrightarrow \pi_{1}\left(C_{*}\right), \\
{\left[c_{0}\right] } & \longmapsto\left\langle c_{0}, c_{0}\right\rangle .
\end{aligned}
$$


In fact $C_{*}$ is stable if and only if the $k$-invariant factors through a homomorphism,

$$
\eta: \pi_{0}\left(C_{*}\right) \otimes \mathbb{Z} / 2 \longrightarrow \pi_{1}\left(C_{*}\right) .
$$

A crossed module morphism $f: C_{*} \rightarrow D_{*}$ is a pair of group homomorphisms $f_{i}: C_{i} \rightarrow D_{i}, i=0,1$, which respect the actions and satisfy $\partial f_{1}=f_{0} \partial$. A reduced or stable 2-module morphism is a morphism $f$ between the underlying crossed modules which preserves the bracket, $\left\langle f_{0}, f_{0}\right\rangle=f_{1}\langle\cdot, \cdot\rangle$.

A homotopy $\alpha: f \Rightarrow g$ between two such morphisms is a function $\alpha: C_{0} \rightarrow D_{1}$ satisfying the equations in Definition 1.4.1. Horizontal and vertical compositions are defined as there. Thus we obtain 2-categories of crossed modules and of reduced and stable 2-modules, together with their morphisms and homotopies of morphisms. All 2-morphisms are invertible, hence we actually have categories enriched in groupoids. Notice that the obvious forgetful 2-functors

stable 2 -modules $\longrightarrow$ reduced 2 -modules $\longrightarrow$ crossed modules

are faithful in dimension 1 and fully faithful in dimension 2, i.e. injective on morphisms and bijective on homotopies.

The 2-category of stable quadratic modules, introduced in Definition 1.4.1, can be identified with the full reflective sub-2-category of the 2-category of stable 2-modules given by those objects $C_{*}$ for which the bracket vanishes whenever one argument lies in the commutator subgroup of $C_{0}$,

$$
\left\langle c_{0},\left[c_{0}^{\prime}, c_{0}^{\prime \prime}\right]\right\rangle=0 .
$$

Compare [MT1, Lemma 4.18].

3.3. Crossed modules and strict categorical groups. The construction $\Gamma$ in Definition 1.4 .4 applied to a braided (resp. symmetric) 2-crossed module $C_{*}$ yields a strict braided (resp. symmetric) categorical group $\Gamma C_{*}$. Moreover, it can also be applied to an ordinary crossed module, producing a categorical group with no braiding. Furthermore, it is also defined on morphisms and homotopies as indicated there. Thus we obtain 2-functors

(reduced, stable 2-)crossed modules $\stackrel{\Gamma}{\longrightarrow}$ strict (braided, symmetric)

cat. groups.

Recall that a strong equivalence of 2-categories is a 2-functor which is fully faithful on 1-morphisms and 2-morphisms, and essentially surjective on objects in the classical sense, i.e. any object in the target is isomorphic, not just equivalent, to an object in the image. 
Proposition 3.3.1. The three 2-functors called $\Gamma$ above are strong equivalences of 2-categories.

Proof: The result is essentially due to Verdier, who discovered the construction $\Gamma$, see $[\mathbf{B S}]$ for some history. To recover a crossed module from a strict categorical group $\mathscr{G}$ is straightforward: $C_{0}$ is the object group, $C_{1}$ is the kernel of target homomorphism, and

$$
\partial(x \stackrel{a}{\longrightarrow} I)=x, \quad(x \stackrel{a}{\longrightarrow} I)^{y}=y^{*} \otimes(x \stackrel{a}{\longrightarrow} I) \otimes y .
$$

A braiding or symmetry also defines a bracket on this crossed module,

$$
\langle x, y\rangle=y^{*} \otimes x^{*} \otimes(y \otimes x \stackrel{\text { comm }}{\longrightarrow} x \otimes y) .
$$

The morphism defined by a strict functor is the obvious one, and a tensor natural transformation $\alpha: f \Rightarrow g$ between strict (symmetric) tensor functors $f, g: \mathscr{G} \rightarrow \mathscr{H}$ yields a homotopy defined by the map $x \mapsto g(x)^{*} \otimes(\alpha(x): f(x) \rightarrow g(x))$.

3.4. Strictifying tensor functors. A strict (braided, symmetric) categorical group is called 0 -free if the group of objects is free. A (reduced, stable 2-)crossed module $C_{*}$ is 0 -free if $C_{0}$ is a free group.

A weak equivalence of 2-categories is a 2-functor which is fully faithful on 2-morphisms, essentially surjective on 1-morphisms, and such that any object in the target is equivalent to an object in the image (in the 2-categorical sense).

In this section we shall prove the following result.

Theorem 3.4.1. The inclusion 2-functor induces a weak equivalence between the 2-categories of:

- 0-free strict (braided, symmetric) categorical groups, strict (symmetric) tensor functors and tensor natural transformations,

- (braided, symmetric) categorical groups, (symmetric) tensor functors and tensor natural transformations.

Obviously the former is a sub-2-category of the latter, full in dimension 2. We give some details of the (folklore) results that (braided, symmetric) categorical groups can be strictified, and that one can replace a strict categorical group by a 0 -free one.

Lemma 3.4.2. Any (braided, symmetric) categorical group is (symmetric) tensor equivalent to a 0 -free strict one. 
Proof: We know that tensor equivalence classes of categorical groups $\mathscr{G}$ with fixed isomorphisms $\pi_{0}(\mathscr{G}) \cong G, \pi_{1}(\mathscr{G}) \cong M$ of groups and $G$-modules, respectively, are in bijection with cohomology classes $H^{3}(G, M)$ [Sin, Chapitre $1 \S 1$, Proposition 10]. We also know that any such class can be represented by a crossed module [Mac1], therefore any categorical group is equivalent to a strict one. In addition a crossed module $C_{*}$ can be replaced by a 0 -free one $D_{*}$ via the pull-back construction

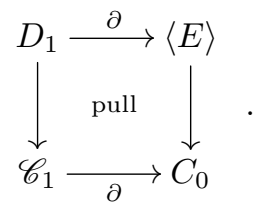

Here $E \subset C_{0}$ is a set of generators of $\pi_{0}\left(C_{*}\right),\langle E\rangle$ is the free group with basis $E$, and $D_{0}=\langle E\rangle \rightarrow C_{0}$ is induced by the inclusion. This commutative square is a morphism of crossed modules which induces isomorphisms on homotopy groups, compare [BM, Proposition 4.15], and therefore an equivalence between the corresponding categorical groups. Notice however that the inverse equivalence need not be strict.

The braided and symmetric case go along the same lines. If $\mathscr{G}$ is braided or symmetric, we can strictify the underlying categorical group and then transfer the symmetry constraint along the equivalence. In this way we obtain an equivalent (braided, symmetric) strict categorical group. The pull-back construction allows us again to replace any reduced or stable 2-module by a 0-free one, compare [BM, Proposition 4.15].

When the source is 0-free, (symmetric) tensor functors can also be strictified. We have not found any reference for the following lemma in the literature.

Lemma 3.4.3. Let $\mathscr{G}$ and $\mathscr{H}$ be strict categorical groups where $\mathscr{G}$ is 0 -free. Then for any tensor functor $\phi: \mathscr{G} \rightarrow \mathscr{H}$ there exists a strict tensor functor $\phi^{s}: \mathscr{G} \rightarrow \mathscr{H}$ together with a tensor natural transformation $\alpha: \phi^{s} \Rightarrow \phi$.

Moreover, if $\phi$ is a symmetric tensor functor between braided or symmetric categorical groups $\mathscr{G}$ and $\mathscr{H}$, then $\phi^{s}$ can be taken to be symmetric.

Proof: Suppose $\mathrm{Ob}(\mathscr{G})$ is free on a set $B$, and define $\phi^{s}: \mathrm{Ob}(\mathscr{G}) \rightarrow$ $\mathrm{Ob}(\mathscr{H})$ to be the unique group homomorphism with $\phi^{s}(b)=\phi(b)$ for $b \in$ $B$. The transformation $\alpha: \phi^{s} \Rightarrow \phi$ is defined on the neutral element $I_{\mathscr{G}}$, 
elements $b \in B$ and products $b \otimes b^{\prime}$, for $b, b^{\prime} \in B$, as follows:

$$
\begin{aligned}
\alpha\left(I_{\mathscr{G}}\right) & =I_{\mathscr{H}} \stackrel{\text { unit }}{\longrightarrow} \phi\left(I_{\mathscr{G}}\right), \\
\alpha(b) & =\phi(b) \stackrel{1}{\longrightarrow} \phi(b), \\
\alpha\left(b \otimes b^{\prime}\right) & =\phi(b) \otimes \phi\left(b^{\prime}\right) \stackrel{\text { mult }_{b, b^{\prime}}}{\longrightarrow} \phi\left(b \otimes b^{\prime}\right) .
\end{aligned}
$$

In general $\alpha$ is defined on objects by induction on the reduced word length in the free group, by the following commutative diagram

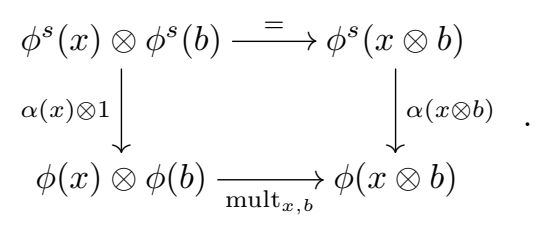

This diagram defines $\alpha(x \otimes b)$ from $\alpha(x)$ provided the last letter in the reduced word $x$ is not $b^{-1}$. At the same, if $x=y \otimes b^{-1}$ is a reduced word, it defines $\alpha\left(y \otimes b^{-1}\right)$ from $\alpha(y)=\alpha(x \otimes b)$. Notice also that this diagram is one case of the condition that $\alpha$ is a tensor natural transformation. The condition is verified in general using induction (on word length of $y$ ) and the following commutative diagram

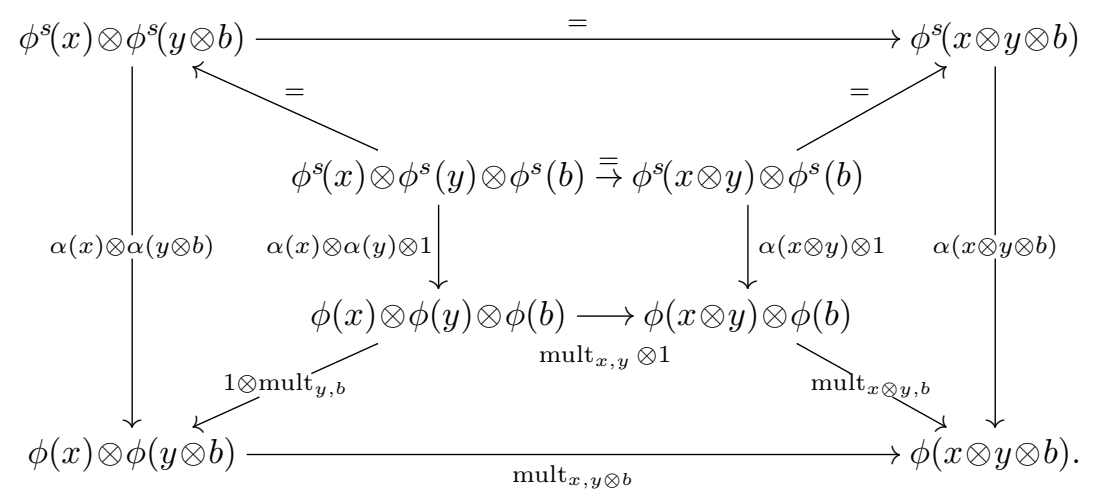

Now $\phi^{s}$ is defined on morphisms $f: x \rightarrow y$ by the following commutative diagram:

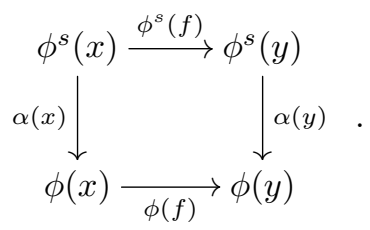

This is just the naturality condition for $\alpha$. 
The following diagram shows the functor $\phi^{s}$ so defined is a tensor functor:

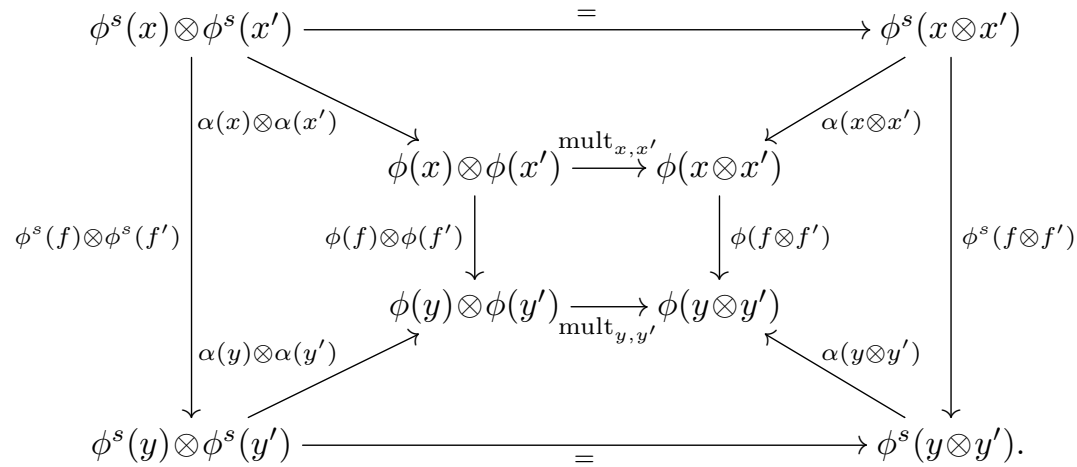

Finally, we note that if $\phi$ is symmetric then so is $\phi^{s}$, by the following commutative diagram:

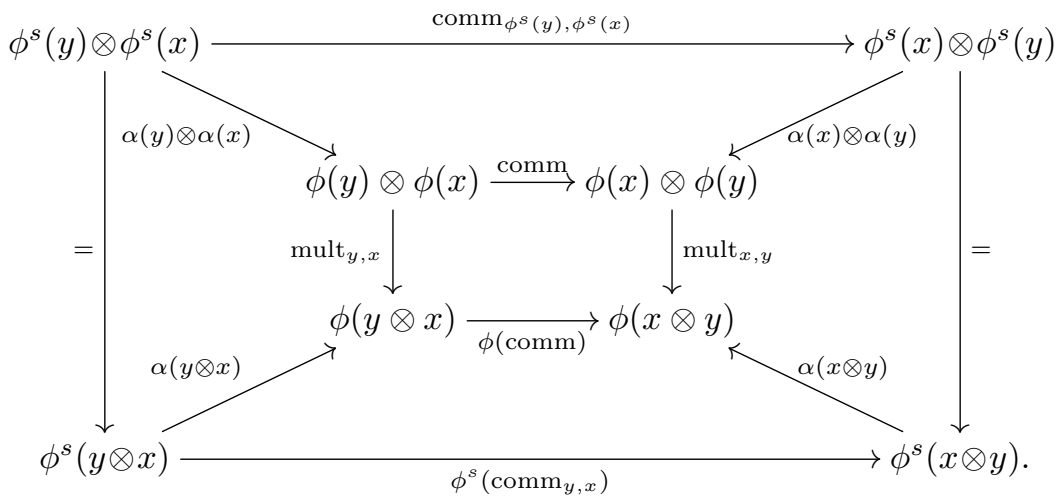

Now Theorem 3.4.1 follows from Lemmas 3.4.2 and 3.4.3.

Proposition 3.4.4. The reflection 2-functor in [MT1, Lemma 4.18] induces a weak equivalence between the 2-categories of 0-free stable 2-modules and 0-free stable quadratic modules.

This follows from [MT1, Remark 4.21]. The following result is a combination of this last result, Proposition 3.3.1, and Theorem 3.4.1.

Corollary 3.4.5. The 2-functor $\Gamma$ in Definition 1.4 .4 induces a weak equivalence between the 2-categories of 0-free stable quadratic modules and Picard groupoids. 


\section{A unified approach to determinant functors}

The motto of category theory is 'one proof replaces many'. With this philosophy in mind, in this section we develop an abstract theory of determinant functors encapsulating all examples in Section 1. In this context, we show the existence of universal determinant functors. We explicitly construct the target, i.e. the category of virtual objects, as the Picard groupoid associated to a stable quadratic module defined by a presentation. These results give our main theorems, stated in Section 1, as corollaries.

The techniques of this section are simplicial. In fact, we only need the low-dimensional part of certain simplicial categories. The strictification results of the previous section are crucial to make the proof of our main results as short as possible. Another advantage is our simple construction of the category of virtual objects.

4.1. Determinant functors for $\boldsymbol{S}_{\bullet}$-categories. Most $K$-theories are defined via a simplicial category, similar to Waldhausen's $S$ • construction. We now define determinant functors for such simplicial categories. This definition generalizes all notions of determinant functors introduced in Section 1.

Definition 4.1.1. An $S_{\bullet}$-category $\mathscr{C}_{\bullet}$ is a simplicial category

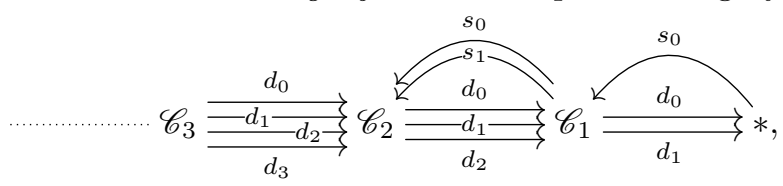

such that $\mathscr{C}_{0}=*$ is the terminal category, with only one object $*$ and one morphism (the identity), $\mathscr{C}_{n}$ has finite coproducts for all $n \geq 0$, and faces and degeneracies preserve coproducts. In particular $s_{0}^{n}(*)$ is initial in $\mathscr{C}_{n}$ for all $n \geq 0$. Moreover, $\mathscr{C}_{\bullet}$ is endowed with a simplicial subcategory we $\mathscr{C}_{\bullet}$ containing all isomorphisms iso $\mathscr{C}_{\bullet} \subset$ we $\mathscr{C}_{\bullet}$, whose morphisms are called weak equivalences. Finite coproducts of weak equivalences are required to be weak equivalences.

Let $\mathscr{P}$ be a Picard groupoid. A determinant functor $\operatorname{det}: \mathscr{C}_{\bullet} \rightarrow \mathscr{P}$ consists of a functor,

$$
\text { det: we } \mathscr{C}_{1} \longrightarrow \mathscr{P} \text {, }
$$

together with additivity data: for any object $\Delta$ in $\mathscr{C}_{2}$, a morphism in $\mathscr{P}$,

$$
\operatorname{det}(\Delta): \operatorname{det}\left(d_{0} \Delta\right) \otimes \operatorname{det}\left(d_{2} \Delta\right) \longrightarrow \operatorname{det}\left(d_{1} \Delta\right),
$$

natural with respect to morphisms in we $\mathscr{C}_{2}$. The following two axioms must be satisfied. 
(1) Associativity: Let $\Theta$ be an object in $\mathscr{C}_{3}$. The following diagram in $\mathscr{P}$ commutes,

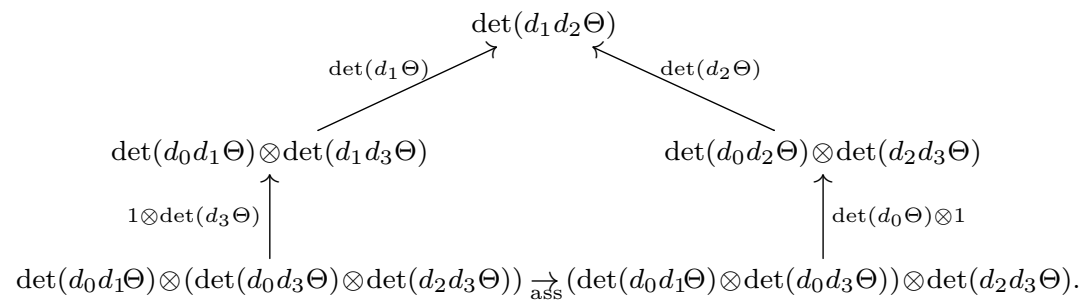

(2) Commutativity: given a coproduct $X \sqcup Y$ of two objects $X$ and $Y$ in $\mathscr{C}_{1}$ the following triangle commutes,

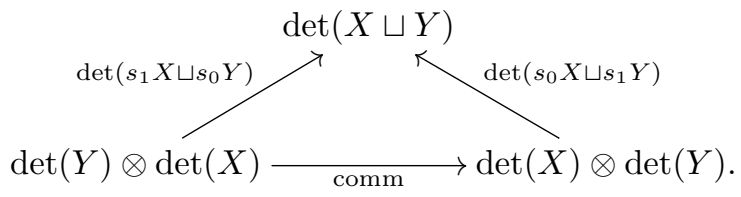

Remark 4.1.2. Notice that in the previous definition we do not use all the structure of $\mathscr{C}_{\bullet}$ but only its 3 -truncation. Actually even less, only the piece of $\mathscr{C}_{\bullet}$ depicted in the diagram at the beginning of Definition 4.1.1. Moreover, we do not use all the structure of that diagram, but just the coproduct operation in we $\mathscr{C}_{1}$ and we $\mathscr{C}_{2}$, the category structure of we $\mathscr{C}_{1}$, the underlying graph of we $\mathscr{C}_{2}$, and the set of objects of $\mathscr{C}_{3}$. This can be illustrated by the following diagram,

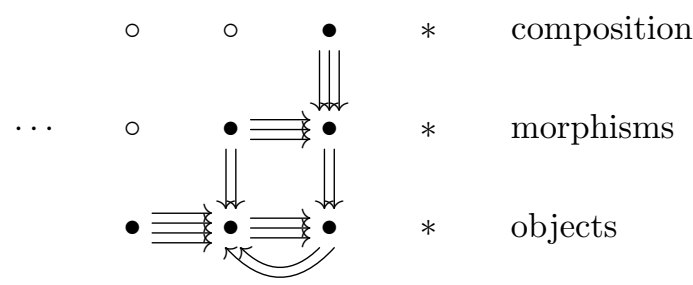

Example 4.1.3. We now see how the determinant functors presented in Section 1 are covered by our unified approach. Weak equivalences are isomorphisms in all $S_{\bullet}$-categories defined below, except for the first one. We need a distinguished zero object for the definition of degeneracies. This is not a real problem, since all zero objects are canonically isomorphic. 
(1) Determinant functors on a Waldhausen category $\mathscr{W}$ coincide with determinant functors on Waldhausen's $S_{\bullet}(\mathscr{W})$ [Wal1, Wal2]. This follows from the fact that $S_{1}(\mathscr{W})$ is just $\mathscr{W}, S_{2}(\mathscr{W})$ is the category of cofiber sequences in $\mathscr{W}, S_{3}(\mathscr{W})$ is the category of staircase diagrams in $\mathscr{W}$, such as (1.2.5), and the non-trivial faces and degeneracies in low dimensions are,

$$
\begin{gathered}
d_{i}\left(X \longleftrightarrow Y \longrightarrow C^{f}\right)= \begin{cases}C^{f}, & i=0 ; \\
Y, & i=1 ; \\
X, & i=2 ;\end{cases} \\
s_{i}(X)= \begin{cases}0 \longleftrightarrow X \stackrel{1}{X} X, \quad i=0 ; \\
X \longleftrightarrow X \longrightarrow 0, \quad i=1 ;\end{cases} \\
d_{i}(1.2 .5)= \begin{cases}C \longmapsto \\
Y \longleftrightarrow C^{f} \longrightarrow C^{g f}, & i=0 ; \\
X \longleftrightarrow C^{g}, & i=1 ; \\
X \longleftrightarrow Z \longrightarrow C^{g f}, & i=2 ;\end{cases}
\end{gathered}
$$

(2) From this description of the low-dimensional part of $S_{\bullet}(\mathscr{W})$, it also follows that derived determinant functors on a Waldhausen category $\mathscr{W}$ coincide with determinant functors on $H_{0} S_{\bullet}(\mathscr{W})$.

(3) Given a triangulated category $\mathscr{T}$ we can consider the 3-truncated $S_{\bullet}$-category $\bar{S}_{\leq 3}\left({ }^{b} \mathscr{T}\right)$,

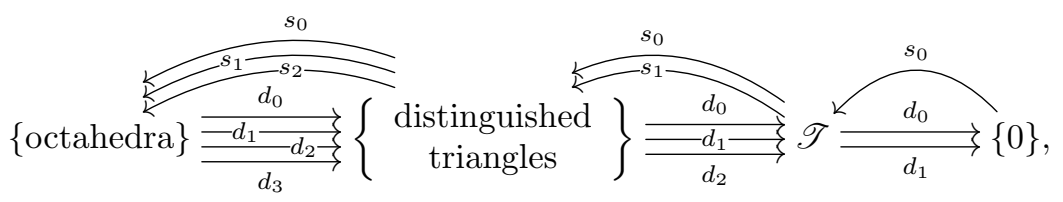

with faces and degeneracies

$$
\begin{gathered}
d_{i}\left(X \stackrel{f}{\longrightarrow} Y \longrightarrow C^{f} \longrightarrow \Sigma X\right)= \begin{cases}C^{f}, & i=0 ; \\
Y, & i=1 ; \\
X, & i=2 ;\end{cases} \\
s_{i}(X)= \begin{cases}0 \longrightarrow X \stackrel{1}{\longrightarrow} X \longrightarrow 0, & i=0 ; \\
X \stackrel{1}{\longrightarrow} X \longrightarrow 0 \longrightarrow \Sigma X, & i=1 ;\end{cases}
\end{gathered}
$$




$$
d_{i}(1.3 .2)= \begin{cases}C^{f} \longrightarrow C^{g f} \longrightarrow C^{g} \longrightarrow \Sigma C^{f}, & i=0 ; \\ Y \stackrel{g}{\longrightarrow} Z \longrightarrow C^{g} \longrightarrow \Sigma Y, & i=1 ; \\ X \stackrel{g f}{\longrightarrow} Z \longrightarrow C^{g f} \longrightarrow \Sigma X, & i=2 ; \\ X \stackrel{f}{\longrightarrow} Y \longrightarrow C^{f} \longrightarrow \Sigma X, & i=3 .\end{cases}
$$

The degeneracies $s_{i}\left(X \stackrel{f}{\rightarrow} Y \rightarrow C^{f} \rightarrow \Sigma X\right), i=0,1,2$, are defined as the unique octahedra with the faces imposed by the simplicial identities.

Breuning determinant functors on $\mathscr{T}$ are the same as determinant functors on $\bar{S}_{\leq 3}\left({ }^{b} \mathscr{T}\right)$.

(4) We can restrict ourselves to special octahedra,

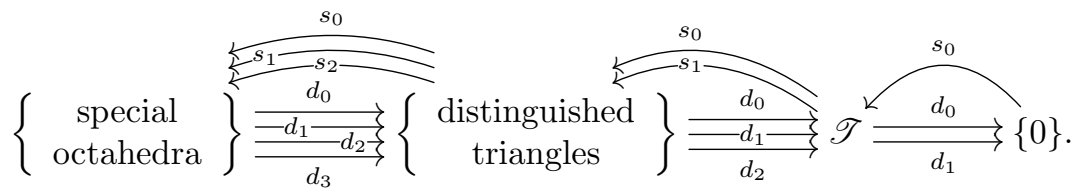

Neeman's $S_{\bullet}\left({ }^{d} \mathscr{T}\right)[$ Nee2] is the simplicial set of objects of an $S_{\bullet}$-category $\bar{S}_{\bullet}(d \mathscr{T})$ whose 3 -truncation is this one. Determinant functors on $\bar{S}_{\bullet}\left({ }^{d} \mathscr{T}\right)$ are special determinant functors on $\mathscr{T}$.

(5) We can also consider virtual triangles and octahedra instead,

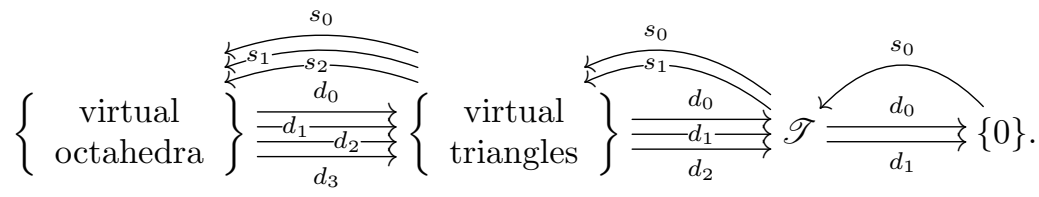

Neeman's $S_{\bullet}(v \mathscr{T})[$ Nee2] is the simplicial set of objects of an $S_{\bullet}$-category $\bar{S}_{\bullet}\left({ }^{v} \mathscr{T}\right)$ whose 3 -truncation is this one. Determinant functors on $\bar{S}_{\bullet}\left({ }^{v} \mathscr{T}\right)$ are virtual determinant functors on $\mathscr{T}$.

(6) Given a strongly triangulated category $\mathscr{T}_{\infty}$ we consider

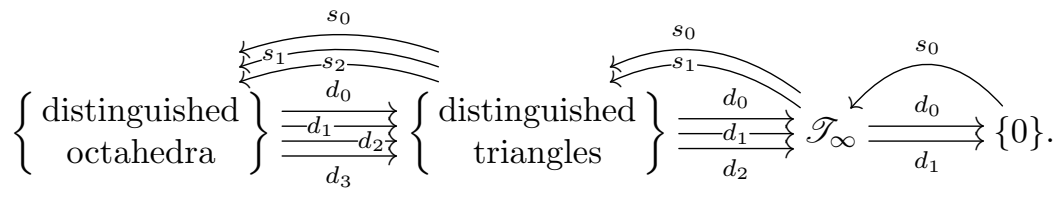

Maltsiniotis's simplicial set $Q_{\bullet}\left(\mathscr{T}_{\infty}\right)[$ Mal1] is the simplicial set of objects of an $S_{\bullet}$-category $\bar{Q}_{\bullet}\left(\mathscr{T}_{\infty}\right)$ whose 3 -truncation is this one. Determinant functors on $\bar{Q} \bullet\left(\mathscr{T}_{\infty}\right)$ are determinant functors on $\mathscr{T}_{\infty}$. 
(7) For an abelian category $\mathscr{A}$, Neeman defined a simplicial set $S_{\bullet}\left(\mathrm{Gr}^{b} \mathscr{A}\right)$ which can be thickened to an $S_{\bullet}$-category $\bar{S}_{\bullet}\left(\mathrm{Gr}^{b} \mathscr{A}\right)$ as in the previous examples, see [Nee2]. The 3-truncation of $\bar{S} \cdot\left(\operatorname{Gr}^{b} \mathscr{A}\right)$ looks as follows:

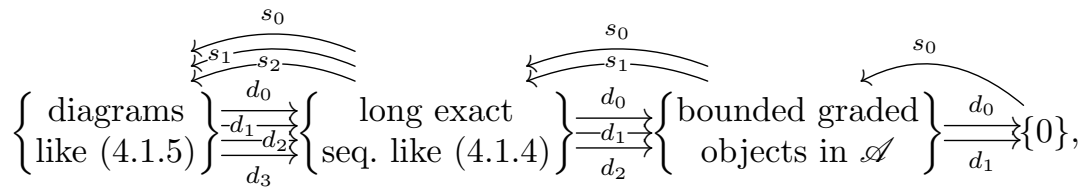

where

$$
\cdots \longrightarrow X_{n} \stackrel{f_{n}}{\longrightarrow} Y_{n} \stackrel{i_{n}}{\longrightarrow} C_{n}^{f} \stackrel{q_{n}}{\longrightarrow} X_{n+1} \longrightarrow \cdots .
$$

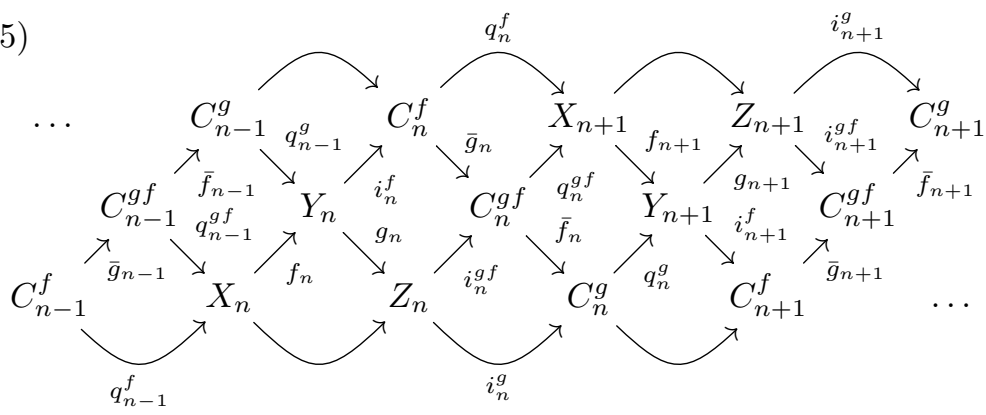

Faces and degeneracies are defined by,

$$
\begin{aligned}
& d_{i}(4.1 .4)= \begin{cases}C_{*}^{f}, & i=0 \\
Y_{*}, & i=1 \\
X_{*}, & i=2\end{cases} \\
& s_{i}(X)= \begin{cases}\cdots \longrightarrow 0 \longrightarrow X_{n} \stackrel{1}{\cdots} X_{n} \longrightarrow 0 \longrightarrow \cdots, & i=0 ; \\
\cdots \longrightarrow X_{n} \stackrel{1}{\longrightarrow} X_{n} \longrightarrow 0 \longrightarrow X_{n+1} \longrightarrow \cdots, & i=1 ;\end{cases} \\
& d_{i}(4.1 .5)= \begin{cases}\cdots \longrightarrow C_{n}^{f} \stackrel{\bar{g}_{n}}{\longrightarrow} C_{n}^{g f} \stackrel{\bar{f}_{n}}{\longrightarrow} C_{n}^{i_{n+1}^{f}} \stackrel{q_{n}^{g}}{\longrightarrow} C_{n+1}^{f} \longrightarrow \cdots, & i=0 ; \\
\cdots \longrightarrow Y_{n} \stackrel{g_{n}}{\longrightarrow} Z_{n} \stackrel{i_{n}^{g}}{\longrightarrow} C_{n}^{g} \stackrel{q_{n}^{g}}{\longrightarrow} Y_{n+1} \longrightarrow \cdots, & i=1 ; \\
\cdots \longrightarrow X_{n} \stackrel{g_{n} f_{n}}{\longrightarrow} Z_{n} \stackrel{i_{n}^{g f}}{\longrightarrow} C_{n}^{g f} \stackrel{q_{n}^{g f}}{\longrightarrow} X_{n+1} \longrightarrow \cdots, & i=2 ; \\
\cdots \longrightarrow X_{n} \stackrel{f_{n}}{\longrightarrow} Y_{n} \stackrel{i_{n}^{f}}{\longrightarrow} C_{n}^{f} \stackrel{q_{n}^{f}}{\longrightarrow} X_{n+1} \longrightarrow \cdots, & i=3 .\end{cases}
\end{aligned}
$$


A graded determinant functor on $\mathscr{A}$ is a determinant functor on $\bar{S}_{\bullet}\left(\operatorname{Gr}^{b} \mathscr{A}\right)$.

(8) The unified approach to determinant functors in Definition 4.1.1 allows us to define determinant functors for triangulated derivators, and more generally for right pointed derivators, using the terminology of [Cis2]. Notice that these are called left pointed derivators in [Gar2, Gar1].

Let Cat be the 2-category of small categories and $\operatorname{Dir}_{f} \subset$ Cat the full sub-2-category of directed finite categories, i.e. those categories whose nerve has a finite number of non-degenerate simplices, e.g. finite posets. The canonical example of derivator is defined from a Waldhausen category $\mathscr{W}$ with cylinders whose weak equivalences satisfy the two-out-of-three axiom, for instance $\mathscr{W}=C^{b}(\mathscr{E})$. It is the contravariant 2-functor

$$
\begin{aligned}
\mathbb{D}_{\mathscr{W}}: \mathbf{D i r}_{f}^{\mathrm{op}} & \longrightarrow \text { Cat }, \\
J & \longmapsto \operatorname{Ho}\left(\mathscr{W}^{J}\right),
\end{aligned}
$$

which takes a directed finite category $J$ to the homotopy category of $J$-indexed diagrams in $\mathscr{W}$. This derivator is regarded as an enhancement of the homotopy category, which arises as a special value of this 2 -functor $\mathbb{D}_{\mathscr{W}}(*)=\operatorname{Ho}(\mathscr{W})$.

For $\mathscr{W}=C^{b}(\mathscr{E}), \mathbb{D}^{b}(\mathscr{E})=\mathbb{D}_{C^{b}(\mathscr{E})}$ is

$$
\begin{aligned}
\mathbb{D}^{b}(\mathscr{E}): \mathbf{D i r}_{f}^{\text {op }} & \longrightarrow \text { Cat, } \\
J & \longmapsto D^{b}\left(\mathscr{E}^{J}\right) .
\end{aligned}
$$

In this case, $\mathbb{D}^{b}(\mathscr{E})(*)=D^{b}(\mathscr{E})$ is the bounded derived category of $\mathscr{E}$.

In general, a right pointed derivator is a 2 -functor $\mathbb{D}: \mathbf{D i r}_{f}^{\mathrm{op}} \rightarrow$ Cat satisfying the formal properties of $\mathbb{D}_{\mathscr{W}}$. Triangulated derivators are modeled after $\mathbb{D}^{b}(\mathscr{E})$.

Garkusha defined in $\left[\right.$ Gar2] a simplicial category $S_{\bullet} \mathbb{D}$. It is only reduced up to the existence of distinct zero objects. We can of course identify different zero objects through the unique isomorphisms between them. This transforms $S_{\bullet} \mathbb{D}$ into an $S_{\bullet}$-category. We define a determinant functor on $\mathbb{D}$ to be a determinant functor on $S_{\bullet}(\mathbb{D})$. The interested reader may work out the explicit definition of determinant functors for right pointed derivators along the lines of Section 1.

Definition 4.1.6. An $S_{\bullet}$-functor is a simplicial functor $f_{\bullet}: \mathscr{C}_{\bullet} \rightarrow \mathscr{C}_{\bullet}^{\prime}$ between $S_{\bullet}$-categories preserving weak equivalences and coproducts. 
Remark 4.1.7. Let $f_{\bullet}: \mathscr{C}_{\bullet} \rightarrow \mathscr{C}_{\bullet}^{\prime}$ be an $S_{\bullet}$-functor and $\operatorname{det}^{\prime}: \mathscr{C}_{\bullet}^{\prime} \rightarrow \mathscr{P}$ a determinant functor. The composite

$$
\operatorname{det}=\operatorname{det}^{\prime} \circ f_{1}: \mathscr{C}_{\bullet} \longrightarrow \mathscr{P}
$$

is a determinant functor on $\mathscr{C}_{\bullet}$ with $\operatorname{det}(\Delta)=\operatorname{det}^{\prime}\left(f_{2}(\Delta)\right)$ for any object $\Delta$ in $\mathscr{C}_{2}$. Actually, it is enough to have a 3 -truncated $S_{\bullet}$-functor $f_{\leq 3}: \mathscr{C}_{\leq 3} \rightarrow \mathscr{C}_{\leq 3}^{\prime}$, and even less, compare Remark 4.1.2.

Example 4.1.8. The following are examples of (3-truncated) $S_{\bullet}$-functors.

(1) Weak equivalences in a Waldhausen category $\mathscr{W}$ project to isomorphisms in the homotopy category, so we have an $S_{\bullet}$-functor

$$
S_{\bullet}(\mathscr{W}) \longrightarrow \operatorname{Ho} S_{\bullet}(\mathscr{W}) \text {. }
$$

(2) In a triangulated category $\mathscr{T}$, any distinguished triangle is virtual, any special octahedron is an ordinary octahedron, and any ordinary octahedron is virtual. This gives rise to 3 -truncated $S_{\bullet}$-functors

$$
\bar{S}_{\leq 3}\left({ }^{d} \mathscr{T}\right) \longrightarrow \bar{S}_{\leq 3}\left({ }^{b} \mathscr{T}\right) \longrightarrow \bar{S}_{\leq 3}\left({ }^{v} \mathscr{T}\right),
$$

which are fully faithful and injective on objects. Actually, the composite is the truncation of an honest $S_{\bullet}$-functor defined in [Nee2],

$$
\bar{S}_{\bullet}\left({ }^{d} \mathscr{T}\right) \longrightarrow \bar{S}_{\bullet}(v \mathscr{T}),
$$

also fully faithful and injective on objects.

(3) If $\mathscr{T}$ is a triangulated category with a $t$-structure, the inclusion of the heart $\mathscr{A} \subset \mathscr{T}$ induces an $S_{\bullet}$-functor $S_{\bullet}(\mathscr{A}) \longrightarrow \bar{S}_{\bullet}(d \mathscr{T})$ fully faithful and injective on objects, see [Nee2].

(4) A strongly triangulated category $\mathscr{T}_{\infty}$ has an underlying triangulated structure. Distinguished octahedra in $\mathscr{T}_{\infty}$ are also ordinary octahedra. Therefore we have a 3 -truncated $S_{\bullet}$-functor which is fully faithful and injective on objects,

$$
\bar{Q}_{\leq 3}\left(\mathscr{T}_{\infty}\right) \longrightarrow \bar{S}_{\leq 3}\left({ }^{b} \mathscr{T}_{\infty}\right) .
$$

(5) If $\mathscr{W}$ is a Waldhausen category with cylinders satisfying the two out of three axiom, there is an $S_{\bullet}$-functor [Gar1, Mur],

$$
\mathrm{Ho}_{\bullet}(\mathscr{W}) \longrightarrow S_{\bullet}\left(\mathbb{D}_{\mathscr{W}}\right) \text {. }
$$

(6) Maltsiniotis indicated in [Mal1] how a triangulated derivator $\mathbb{D}$ induces a strongly triangulated structure on $\mathbb{D}(*)$. There is an $S_{\bullet}$-functor

$$
S_{\bullet}(\mathbb{D}) \longrightarrow \bar{Q} \bullet(\mathbb{D}(*))
$$

defined by using the canonical evaluation functors from $\mathbb{D}(J)$ to the category of functors $J \rightarrow \mathbb{D}(*)$. 
(7) If $\mathscr{E}$ is an exact category, the inclusion of complexes concentrated in degree $0, \mathscr{E} \subset C^{b}(\mathscr{E})$, induces an $S_{\bullet}$-functor

$$
S_{\bullet}(\mathscr{E}) \longrightarrow S_{\bullet}\left(C^{b}(\mathscr{E})\right) \text {. }
$$

(8) Let $\mathscr{E}$ be again an exact category. The $S_{\bullet}$-functor

$$
S_{\bullet}\left(C^{b}(\mathscr{E})\right) \longrightarrow \bar{Q}_{\bullet}\left(D^{b}(\mathscr{E})\right)
$$

given by (1), (5), and (6) can be directly described in terms of the canonical functor $C^{b}(\mathscr{E}) \rightarrow D^{b}(\mathscr{E})$, compare [Mal1].

(9) Let $\mathscr{A}$ be an abelian category, the inclusion of objects concentrated in degree 0 is an $S_{\bullet}$-functor $S_{\bullet}(\mathscr{A}) \rightarrow \bar{S}_{\bullet}\left(\operatorname{Gr}^{b} \mathscr{A}\right)$.

4.2. The groupoid of determinant functors. In this section we introduce universal determinant functor for $S_{\bullet}$-categories.

Definition 4.2.1. Let $\mathscr{C}_{\bullet}$ be an $S_{\bullet}$-category and $\mathscr{P}$ a Picard groupoid. Given determinant functors det, $\operatorname{det}^{\prime}: \mathscr{C}_{\bullet} \rightarrow \mathscr{P}$, a morphism $f: \operatorname{det} \rightarrow$ $\operatorname{det}^{\prime}$ is a natural transformation between the underlying functors det, det $^{\prime}:$ we $\mathscr{C}_{1} \rightarrow \mathscr{P}$ compatible with the additivity data, i.e. given an object $\Delta$ in $\mathscr{C}_{2}$, the following diagram commutes:

$$
\begin{aligned}
\operatorname{det}\left(d_{0} \Delta\right) & \otimes \operatorname{det}\left(d_{2} \Delta\right) \stackrel{\operatorname{det}(\Delta)}{\longrightarrow} \operatorname{det}\left(d_{1} \Delta\right) \\
f\left(d_{0} \Delta\right) \otimes f\left(d_{2} \Delta\right) & \downarrow \\
\operatorname{det}^{\prime}\left(d_{0} \Delta\right) & \otimes \operatorname{det}^{\prime}\left(d_{2} \Delta\right) \underset{f\left(d_{1} \Delta\right)}{\stackrel{\downarrow}{\operatorname{det}^{\prime}(\Delta)} \operatorname{det}^{\prime}\left(d_{1} \Delta\right)} .
\end{aligned}
$$

Notice that all these morphisms are invertible since $\mathscr{P}$ is a groupoid. The resulting groupoid of determinant functors is denoted by $\operatorname{Det}\left(\mathscr{C}_{\bullet}, \mathscr{P}\right)$.

Remark 4.2.2. The category $\operatorname{Det}\left(\mathscr{C}_{\bullet}, \mathscr{P}\right)$ is itself a Picard groupoid. The tensor structure is given as follows. For any determinant functors det,

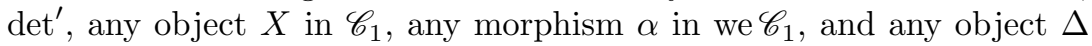
in $\mathscr{C}_{2}$, we define:

$$
\begin{aligned}
\left(\operatorname{det} \otimes \operatorname{det}^{\prime}\right)(X) & =\operatorname{det}(X) \otimes \operatorname{det}^{\prime}(X), \\
\left(\operatorname{det} \otimes \operatorname{det}^{\prime}\right)(\alpha) & =\operatorname{det}(\alpha) \otimes \operatorname{det}^{\prime}(\alpha), \\
\left(\operatorname{det} \otimes \operatorname{det}^{\prime}\right)(\Delta) & =\left(\operatorname{det}(\Delta) \otimes \operatorname{det}^{\prime}(\Delta)\right) \circ(1 \otimes \operatorname{comm} \otimes 1) .
\end{aligned}
$$

The unit object is the constant determinant functor, which sends all objects in $\mathscr{C}_{1}$ to the tensor unit $I$ of $\mathscr{P}$, all weak equivalences in $\mathscr{C}_{1}$ to the identity on $I$, and all objects in $\mathscr{C}_{2}$ to the isomorphism $I \otimes I \cong I$. 
Associativity, commutativity, and unit constraints are pointwise defined by the corresponding constraints in $\mathscr{P}$. This structure has already been considered in [Knu1, Proposition 1.13] for determinant functors on exact categories.

Definition 4.2 .3 . Let $\mathscr{C}_{\bullet}$ be an $S_{\bullet}$-category. The composition of a determinant functor and a symmetric tensor functor is again a determinant functor,

$$
\mathscr{C}_{\bullet} \stackrel{\operatorname{det}}{\longrightarrow} \mathscr{P} \stackrel{f}{\longrightarrow} \mathscr{P}^{\prime} \text {. }
$$

The underlying functor $f \circ \operatorname{det}:$ we $\mathscr{C}_{1} \rightarrow \mathscr{P}^{\prime}$ is the usual composition, and additivity data $(f \circ \operatorname{det})(\Delta)$ are defined by

$$
\begin{aligned}
& f\left(\operatorname{det}\left(d_{0} \Delta\right)\right) \otimes f\left(\operatorname{det}\left(d_{2} \Delta\right)\right) \stackrel{\text { mult }}{\longrightarrow} f\left(\operatorname{det}\left(d_{0} \Delta\right) \otimes \operatorname{det}\left(d_{2} \Delta\right)\right) \\
& \stackrel{f(\operatorname{det}(\Delta))}{\longrightarrow} f\left(\operatorname{det}\left(d_{1} \Delta\right)\right) \text {. }
\end{aligned}
$$

Moreover, a tensor natural transformation $\alpha: f \Rightarrow g$ as in the following diagram

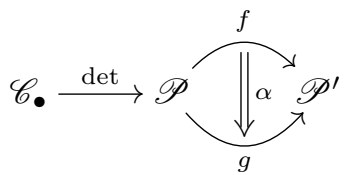

induces a morphism of determinant functors $\alpha \circ \operatorname{det}: f \circ \operatorname{det} \Rightarrow g \circ$ det defined as the usual horizontal composition of functors and natural transformations. This composite is compatible with additivity data in the sense of Definition 4.2.1.

All this shows that the Picard groupoid $\operatorname{Det}\left(\mathscr{C}_{\bullet}, \mathscr{P}\right)$ is 2 -functorial in $\mathscr{P}$, i.e. it defines a 2-functor from the 2-category of Picard groupoids to the 2-category of groupoids. Actually, the target can be taken to be the 2-category of Picard groupoids again, along the lines of the previous remark. In particular, a determinant functor det: $\mathscr{C}_{\bullet} \rightarrow \mathscr{P}$ gives rise to a functor

$$
\text { - o det: } \operatorname{Hom}_{c}^{\otimes}\left(\mathscr{P}, \mathscr{P}^{\prime}\right) \longrightarrow \operatorname{Det}\left(\mathscr{C}_{\bullet}, \mathscr{P}^{\prime}\right) .
$$

A determinant functor det: $\mathscr{C}_{\bullet} \rightarrow V\left(\mathscr{C}_{\bullet}\right)$ is universal if

$$
\text { - o det: } \operatorname{Hom}_{c}^{\otimes}\left(V\left(\mathscr{C}_{\bullet}\right), \mathscr{P}\right) \longrightarrow \operatorname{Det}\left(\mathscr{C}_{\bullet}, \mathscr{P}\right)
$$

is an equivalence of categories for any Picard groupoid $\mathscr{P}$. In this situation, we say that $V\left(\mathscr{C}_{\bullet}\right)$ is the category of virtual objects of $\mathscr{C}$. This Picard groupoid is well defined up to equivalence, since it represents the 2-functor $\operatorname{Det}\left(\mathscr{C}_{\bullet},-\right)$. 
Remark 4.2.5. Universal determinant functors on $S_{\bullet}$-categories can also be characterized by a 2-categorical universal property along the lines of Definition 1.2.9. In particular, the various notions of universal determinant functor introduced in Section 1 are consistent with Definition 4.2.3 and Example 4.1.3. The more abstract approach in Definition 4.2 .3 will be helpful in the proof of the existence of universal determinant functors.

For determinant functors with values in strict Picard groupoids it is convenient to introduce also the following notion.

Definition 4.2.6. A determinant functor det: $\mathscr{C}_{\bullet} \rightarrow \mathscr{P}$ with values in a strict Picard groupoid $\mathscr{P}$ is strict if it satisfies

$$
\operatorname{det}\left(s_{0}(*)\right)=I, \quad \operatorname{det}\left(s_{0}^{2}(*)\right)=1_{I} .
$$

We denote by $\operatorname{Det}_{s}\left(\mathscr{C}_{\bullet}, \mathscr{P}\right)$ the full subcategory of $\operatorname{Det}\left(\mathscr{C}_{\bullet}, \mathscr{P}\right)$ whose objects are the strict determinant functors.

Lemma 4.2.7. The inclusion $\operatorname{Det}_{s}\left(\mathscr{C}_{\bullet}, \mathscr{P}\right) \subset \operatorname{Det}\left(\mathscr{C}_{\bullet}, \mathscr{P}\right)$ is an equivalence, natural in the strict Picard groupoid $\mathscr{P}$.

Proof: Let det: $\mathscr{C}_{\bullet} \rightarrow \mathscr{P}$ be a determinant functor. Then we can define a strict determinant functor $\operatorname{det}^{\prime}$ by

$$
\operatorname{det}^{\prime}=\operatorname{det} \otimes \operatorname{det}\left(s_{0}(*)\right)^{-1}: \text { we } \mathscr{C}_{1} \longrightarrow \mathscr{P}
$$

and by

$\operatorname{det}^{\prime}(\Delta)=\left(\operatorname{det}(\Delta) \otimes 1_{\operatorname{det}\left(s_{0}(*)\right)^{-1}}\right) \circ\left(\operatorname{det}\left(s_{0} d_{0} \Delta\right)^{-1} \otimes 1_{\operatorname{det}\left(s_{0}(*)\right)^{-1} \otimes \operatorname{det}^{\prime}\left(d_{2} \Delta\right)}\right)$ for objects $\Delta$ in $\mathscr{C}_{2}$. Moreover, $X \mapsto \operatorname{det}\left(s_{0} X\right) \otimes 1_{\operatorname{det}\left(s_{0}(*)\right)^{-1}}$ defines a morphism of determinant functors det $\rightarrow$ det $^{\prime}$.

4.3. The existence of universal determinant functors. In this section we will show that universal determinant functors always exist, which is our main result. We will actually construct universal determinant functors by using presentations of stable quadratic modules.

Definition 4.3.1. Let $\mathscr{C}_{\bullet}$ be an $S_{\bullet}$-category. We define the stable quadratic module $\mathcal{D}_{*}\left(\mathscr{C}_{\bullet}\right)$ by generators,

(G1) $[X]$ for any object in $\mathscr{C}_{1}$, in dimension 0 ,

(G2) $\left[X \stackrel{\sim}{\rightarrow} X^{\prime}\right]$ for any weak equivalence in $\mathscr{C}_{1}$, in dimension 1 ,

(G3) $[\Delta]$ for any object in $\mathscr{C}_{2}$, in dimension 1 ,

and relations,

(R1) $\partial\left[X \stackrel{\sim}{\rightarrow} X^{\prime}\right]=-\left[X^{\prime}\right]+[X]$,

(R2) $\partial[\Delta]=-\left[d_{1} \Delta\right]+\left[d_{0} \Delta\right]+\left[d_{2} \Delta\right]$, 
(R3) $\left[s_{0}(*)\right]=0$ for the degenerate object of $\mathscr{C}_{1}$,

(R4) $[X \stackrel{1}{\rightarrow} X]=0$, for all identity morphisms in $\mathscr{C}_{1}$,

(R5) $\left[s_{0} X\right]=0=\left[s_{1} X\right]$ for any object $X$ in $\mathscr{C}_{1}$,

(R6) for any pair of composable weak equivalences $X \stackrel{\sim}{\rightarrow} Y \stackrel{\sim}{\rightarrow} Z$ in $\mathscr{C}_{1}$,

$$
[X \stackrel{\sim}{\longrightarrow} Z]=[Y \stackrel{\sim}{\longrightarrow} Z]+[X \stackrel{\sim}{\longrightarrow} Y],
$$

(R7) for any weak equivalence $\Phi: \Delta \stackrel{\sim}{\rightarrow} \Delta^{\prime}$ in $\mathscr{C}_{2}$,

$$
\left[d_{2} \Phi\right]+\left[d_{0} \Phi\right]^{\left[d_{2} \Delta\right]}=-\left[\Delta^{\prime}\right]+\left[d_{1} \Phi\right]+[\Delta]
$$

(R8) for any object $\Theta$ in $\mathscr{C}_{3}$,

$$
\left[d_{1} \Theta\right]+\left[d_{3} \Theta\right]=\left[d_{2} \Theta\right]+\left[d_{0} \Theta\right]^{\left[d_{2} d_{3} \Theta\right]},
$$

(R9) for any two objects $X$ and $Y$ in $\mathscr{C}_{1}$,

$$
\langle[X],[Y]\rangle=-\left[s_{0} X \sqcup s_{1} Y\right]+\left[s_{1} X \sqcup s_{0} Y\right] .
$$

Remark 4.3.2. This is not a minimal presentation, compare [MT2, Remark 1.4], but it is the most intuitive. Relation (R4) follows from (R6). Relation (R3) follows from (R5),

$$
0=\partial\left[s_{1} X\right]=-[X]+[X]+\left[s_{0}(*)\right]=\left[s_{0}(*)\right] .
$$

Relation (R5) is equivalent to imposing $\left[s_{0}^{2}(*)\right]=0$ for the degenerate object of $\mathscr{C}_{2}$. Indeed, applying (R8) to $s_{0}^{2} X$ and $s_{1}^{2} X$, respectively, we obtain,

$$
\begin{aligned}
{\left[s_{0} X\right]+\left[s_{0}^{2}(*)\right] } & =\left[s_{0} X\right]+\left[s_{0} X\right]^{\left[s_{0}(*)\right]}, \\
{\left[s_{1} X\right]+\left[s_{1} X\right] } & =\left[s_{1} X\right]+\left[s_{0}^{2}(*)\right]^{[X]} .
\end{aligned}
$$

Remark 4.3.3. The stable quadratic module $\mathcal{D}_{*}\left(\mathscr{C}_{\bullet}\right)$ is functorial with respect to $S_{\bullet}$-functors,

$$
\begin{aligned}
& \mathcal{D}_{0}\left(f_{\bullet}\right): \mathcal{D}_{0}\left(\mathscr{C}_{\bullet}\right) \longrightarrow \mathcal{D}_{0}\left(\mathscr{C}_{\bullet}^{\prime}\right), \\
& {[X] \longmapsto\left[f_{1}(X)\right],} \\
& \mathcal{D}_{1}\left(f_{\bullet}\right): \mathcal{D}_{1}\left(\mathscr{C}_{\bullet}\right) \longrightarrow \mathcal{D}_{1}\left(\mathscr{C}_{\bullet}^{\prime}\right), \\
& {\left[\phi: X \stackrel{\sim}{\longrightarrow} X^{\prime}\right] \longmapsto\left[f_{1}(\phi): f_{1}(X) \stackrel{\sim}{\longrightarrow} f_{1}\left(X^{\prime}\right)\right],} \\
& {[\Delta] \longmapsto\left[f_{2}(\Delta)\right] .}
\end{aligned}
$$

Moreover, it is 2-functorial with respect to simplicial natural weak equivalences $\alpha_{\bullet}: f_{\bullet} \Rightarrow g_{\bullet}$ between $S_{\bullet}$-functors $f_{\bullet}, g_{\bullet}: \mathscr{C}_{\bullet} \rightarrow \mathscr{C}_{\bullet}$, i.e. simplicial 
natural transformations taking values in the subcategories of weak equivalences,

$$
\begin{aligned}
\mathcal{D}_{*}\left(\alpha_{\bullet}\right): \mathcal{D}_{0}\left(\mathscr{C}_{\bullet}\right) & \longrightarrow \mathcal{D}_{1}\left(\mathscr{C}_{\bullet}^{\prime}\right), \\
{[X] \longmapsto } & {\left[\alpha_{1}(X): f_{1}(X) \stackrel{\sim}{\longrightarrow} g_{1}(X)\right] . }
\end{aligned}
$$

Actually, it is 2-functorial at the 3 -truncated level.

If we evaluate $\mathcal{D}_{*}\left(\mathscr{C}_{\bullet}\right)$ at the $S_{\bullet}$-categories in Example $4.1 .3(1-6)$ we obtain the stable quadratic modules in Definition 1.5.1.

Theorem 4.3.4. There is a universal determinant functor det: $\mathscr{C}_{\bullet} \rightarrow$ $\Gamma \mathcal{D}_{*}\left(\mathscr{C}_{\bullet}\right)$ defined by

- $\operatorname{det}(X)=[X]$ for any object in $\mathscr{C}_{1}$,

- $\operatorname{det}\left(X \stackrel{\sim}{\rightarrow} X^{\prime}\right)=\left(\left[X^{\prime}\right],\left[X \stackrel{\sim}{\rightarrow} X^{\prime}\right]\right)$ for any weak equivalence in $\mathscr{C}_{1}$,

- $\operatorname{det}(\Delta)=\left(\left[d_{1} \Delta\right],[\Delta]\right)$ for any object in $\mathscr{C}_{2}$.

This determinant functor is strict. Moreover, for any stable quadratic module $C_{*}$, the functor

$$
\begin{aligned}
\operatorname{hom}\left(\mathcal{D}_{*}\left(\mathscr{C}_{\bullet}\right), C_{*}\right) & \longrightarrow \operatorname{Det}_{s}\left(\mathscr{C}_{\bullet}, \Gamma C_{*}\right) \\
\varphi & \longmapsto(\Gamma \varphi) \circ \operatorname{det}
\end{aligned}
$$

is an isomorphism of groupoids. Here the source is a morphism groupoid in the 2-category of stable quadratic modules.

Proof: A strict determinant functor det: $\mathscr{C}_{\bullet} \rightarrow \Gamma C_{*}$ sends an object $X$ in $\mathscr{C}_{1}$, a weak equivalence $f: X \stackrel{\sim}{\rightarrow} X^{\prime}$ in $\mathscr{C}_{1}$, and an object $\Delta$ in $\mathscr{C}_{2}$ to elements

$$
\begin{gathered}
\operatorname{det}(X) \in C_{0}, \\
\operatorname{det}(f)=\left(\operatorname{det}\left(X^{\prime}\right),\{f\}\right) \in C_{0} \ltimes C_{1}, \\
\operatorname{det}(\Delta)=\left(\operatorname{det}\left(d_{1} \Delta\right),\{\Delta\}\right) \in C_{0} \ltimes C_{1} .
\end{gathered}
$$

The first coordinate of $\operatorname{det}(f)$ is $\operatorname{det}\left(X^{\prime}\right)$ since it is the target. The source is $\operatorname{det}\left(X^{\prime}\right)+\partial\{f\}=\operatorname{det}(X)$, i.e.

$$
\partial\{f\}=-\operatorname{det}\left(X^{\prime}\right)+\operatorname{det}(X) .
$$

For the same reasons, the first coordinate of $\operatorname{det}(\Delta)$ is $\operatorname{det}\left(d_{1} \Delta\right)$ and

$$
\partial\{\Delta\}=-\operatorname{det}\left(d_{1} \Delta\right)+\operatorname{det}\left(d_{0} \Delta\right)+\operatorname{det}\left(d_{2} \Delta\right) .
$$

The functor det preserves identities, $\operatorname{det}\left(1_{X}\right)=1_{\operatorname{det}(X)}=(\operatorname{det}(X), 0)$, i.e.

$$
\left\{1_{X}\right\}=0 .
$$


Moreover, it also preserves compositions. Since

$$
\begin{aligned}
\operatorname{det}(g f: X \stackrel{f}{\longrightarrow} Y \stackrel{g}{\longrightarrow} Z) & =(\operatorname{det}(Z),\{g f\}), \\
\operatorname{det}(g) \circ \operatorname{det}(f) & =(\operatorname{det}(Z),\{g\}) \circ(\operatorname{det}(Y),\{f\}) \\
& =(\operatorname{det}(Z),\{g\}+\{f\}),
\end{aligned}
$$

then

$$
\{g f\}=\{g\}+\{f\} .
$$

Naturality of additivity data with respect to weak equivalences $\Phi: \Delta \stackrel{\sim}{\rightarrow}$ $\Delta^{\prime}$ in $\mathscr{C}_{2}$ says that the following diagram in $\Gamma C_{*}$ must commute,

$$
\begin{aligned}
& \operatorname{det}\left(d_{0} \Delta\right)+\operatorname{det}\left(d_{2} \Delta\right) \stackrel{\operatorname{det}(\Delta)=\left(\operatorname{det}\left(d_{1} \Delta\right),\{\Delta\}\right)}{\longrightarrow} \operatorname{det}\left(d_{1} \Delta\right) \\
& \qquad \begin{array}{l}
\operatorname{det}\left(d_{0} \Phi\right)+\operatorname{det}\left(d_{2} \Phi\right) \\
=\left(\operatorname{det}\left(d_{0} \Delta^{\prime}\right),\left\{d_{0} \Phi\right\}\right)+\left(\operatorname{det}\left(d_{2} \Delta^{\prime}\right),\left\{d_{2} \Phi\right\}\right) \\
=\left(\operatorname{det}\left(d_{0} \Delta^{\prime}\right)+\operatorname{det}\left(d_{2} \Delta^{\prime}\right),\left\{d_{0} \Phi\right\}^{\operatorname{det}\left(d_{2} \Delta^{\prime}\right)}+\left\{d_{2} \Phi\right\}\right)
\end{array} \\
& \downarrow \\
& \operatorname{det}\left(d_{0} \Delta^{\prime}\right)+\operatorname{det}\left(d_{2} \Delta^{\prime}\right) \underset{\operatorname{det}\left(d_{1} \Phi\right)}{=\left(\operatorname{det}\left(d_{1} \Delta^{\prime}\right),\left\{d_{1} \Phi\right\}\right)}
\end{aligned}
$$

i.e.

$$
\begin{aligned}
\left\{d_{1} \Phi\right\}+\{\Delta\} & =\left\{\Delta^{\prime}\right\}+\left\{d_{0} \Phi\right\}^{\operatorname{det}\left(d_{2} \Delta^{\prime}\right)}+\left\{d_{2} \Phi\right\} \\
& =\left\{\Delta^{\prime}\right\}+\left\{d_{2} \Phi\right\}+\left\{d_{0} \Phi\right\}^{\operatorname{det}\left(d_{2} \Delta^{\prime}\right)+\partial\left\{d_{2} \Phi\right\}} \\
& =\left\{\Delta^{\prime}\right\}+\left\{d_{2} \Phi\right\}+\left\{d_{0} \Phi\right\}^{\operatorname{det}\left(d_{2} \Delta\right)} .
\end{aligned}
$$

The Picard groupoid $\Gamma C_{*}$ is strict, in particular associativity constraints are identities. Hence the associativity axiom says that, for any object $\Theta$ in $\mathscr{C}_{3}$, the following diagram commutes

$$
\begin{aligned}
& \operatorname{det}\left(d_{0} d_{1} \Theta\right)+\operatorname{det}\left(d_{1} d_{3} \Theta\right) \stackrel{\operatorname{det}\left(d_{1} \Theta\right)}{=\left(\operatorname{det}\left(d_{1} d_{2} \Theta\right),\left\{d_{1} \Theta\right\}\right)} \longrightarrow \operatorname{det}\left(d_{1} d_{2} \Theta\right)
\end{aligned}
$$

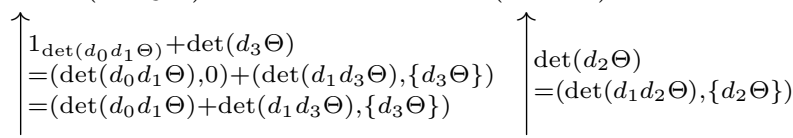

$$
\begin{aligned}
& \operatorname{det}\left(d_{0} d_{1} \Theta\right)+\operatorname{det}\left(d_{0} d_{3} \Theta\right)+\operatorname{det}\left(d_{2} d_{3} \Theta\right) \rightarrow \operatorname{det}\left(d_{0} d_{2} \Theta\right)+\operatorname{det}\left(d_{2} d_{3} \Theta\right) \\
& \operatorname{det}\left(d_{0} \Theta\right)+1_{\operatorname{det}\left(d_{2} d_{3} \Theta\right)} \\
& =\left(\operatorname{det}\left(d_{0} d_{2} \Theta\right),\left\{d_{0} \Theta\right\}\right)+\left(\operatorname{det}\left(d_{2} d_{3} \Theta\right), 0\right) \\
& =\left(\operatorname{det}\left(d_{0} d_{2} \Theta\right)+\operatorname{det}\left(d_{2} d_{3} \Theta\right),\left\{d_{0} \Theta\right\}^{\operatorname{det}\left(d_{2} d_{3} \Theta\right)}\right)
\end{aligned}
$$

i.e.

$$
\left\{d_{1} \Theta\right\}+\left\{d_{3} \Theta\right\}=\left\{d_{2} \Theta\right\}+\left\{d_{0} \Theta\right\}^{\operatorname{det}\left(d_{2} d_{3} \Theta\right)} .
$$


The commutativity axiom says that the following diagram commutes for any pair of objects $X$ and $Y$ in $\mathscr{C}_{1}$,

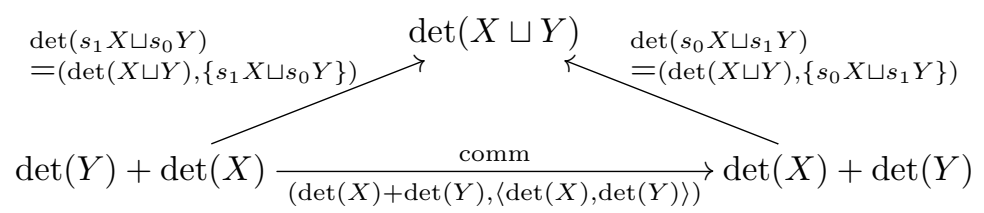

i.e.

$$
\left\{s_{0} X \sqcup s_{1} Y\right\}+\langle\operatorname{det}(X), \operatorname{det}(Y)\rangle=\left\{s_{1} X \sqcup s_{0} Y\right\} .
$$

Being strict means that $\operatorname{det}\left(s_{0}(*)\right)=I=0$ and $\operatorname{det}\left(s_{0}^{2}(*)\right)=1_{I}=$ $(0,0)$, i.e.

$$
\operatorname{det}\left(s_{0}(*)\right)=0, \quad\left\{s_{0}^{2}(*)\right\}=0 .
$$

Conversely, any choice of elements

$$
\operatorname{det}(X) \in C_{0}, \quad\{f\} \in C_{1}, \quad\{\Delta\} \in C_{1},
$$

satisfying the previous equations yields a strict determinant functor $\operatorname{det}: \mathscr{C}_{\bullet} \rightarrow \Gamma C_{*}$ defined by (4.3.5).

This, combined with the presentation of $\mathcal{D}_{*}\left(\mathscr{C}_{\bullet}\right)$ in Definition 4.3.1 and the alternative set of relations in Remark 4.3.2, has two important consequences: the formulas in the statement define a strict determinant functor det: $\mathscr{C}_{\bullet} \rightarrow \Gamma \mathcal{D}_{*}\left(\mathscr{C}_{\bullet}\right)$, and any strict determinant functor $\operatorname{det}^{\prime}: \mathscr{C}_{\bullet} \rightarrow \Gamma C_{*}$ factors uniquely as

$$
\operatorname{det}^{\prime}: \mathscr{C}_{\bullet} \stackrel{\operatorname{det}}{\longrightarrow} \Gamma \mathcal{D}_{*}\left(\mathscr{C}_{\bullet}\right) \stackrel{\Gamma \varphi}{\longrightarrow} \Gamma C_{*},
$$

where $\varphi: \mathcal{D}_{*}\left(\mathscr{C}_{\bullet}\right) \rightarrow C_{*}$ is a morphism of stable quadratic modules. Hence, it is only left to check that det: $\mathscr{C}_{\bullet} \rightarrow \Gamma \mathcal{D}_{*}\left(\mathscr{C}_{\bullet}\right)$ is universal.

Let $\mathscr{P}$ be any Picard groupoid. By Corollary 3.4.5, there exists a 0 -free stable quadratic module $C_{*}$ and an equivalence $f: \Gamma C_{*} \stackrel{\sim}{\rightarrow} \mathscr{P}$ in the 2-category of Picard groupoids. Consider the following commutative diagram

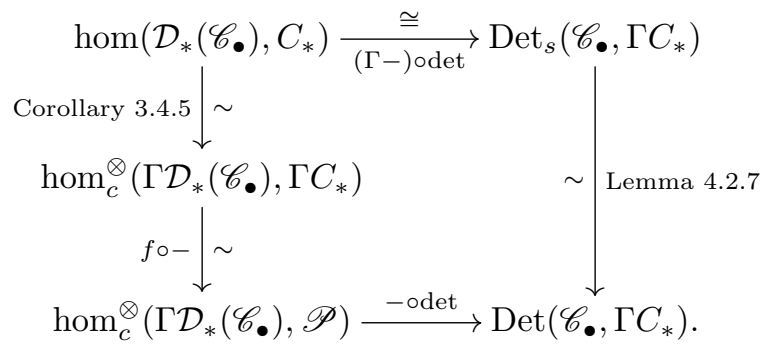


The upper horizontal arrow is an isomorphism. This is the last part of the statement, that we have just checked. Two vertical arrows are equivalences by the results indicated in the labels. Moreover, $f \circ-$ is an equivalence of categories since $f$ is an equivalence of Picard groupoids. Thus the lower horizontal arrow is also an equivalence.

4.4. Non-commutative determinant functors. In [Del], Deligne also considers determinant functors into categorical groups that are not necessarily symmetric. Of course, one has to omit the commutativity axiom in Definition 4.1.1 if one chooses to work in this context. We will call those determinant functors non-commutative determinant functors. However, as Deligne already noticed, it turns out that this notion is not essentially more general that the theory of commutative determinant functors considered above.

We consider the left adjoint of the functor sending a crossed module $C_{*}$ to the pair of sets $\left(C_{0}, C_{1}\right)$. Objects in the image of this left adjoint are said to be free.

Let $\langle E\rangle$ denote the free group on a set $E$. The free crossed module $F_{*}^{c}\left(E_{0}, E_{1}\right)$ on a pair of sets $\left(E_{0}, E_{1}\right)$ is defined as follows: $F_{0}^{c}\left(E_{0}, E_{1}\right)=$ $\left\langle E_{0} \sqcup E_{1}\right\rangle$ is a free group, $F_{1}^{c}\left(E_{0}, E_{1}\right)=\operatorname{ker} p$ is the kernel of the homomorphism,

$$
\left\langle E_{0} \sqcup E_{1}\right\rangle \stackrel{p}{\longrightarrow}\left\langle E_{0}\right\rangle, \quad E_{0} \ni e_{0} \longmapsto e_{0}, \quad E_{1} \ni e_{1} \longmapsto 0,
$$

the homomorphism $\partial: F_{1}^{c}\left(E_{0}, E_{1}\right) \hookrightarrow F_{0}^{c}\left(E_{0}, E_{1}\right)$ is the inclusion, and $F_{0}^{c}\left(E_{0}, E_{1}\right)$ acts on $F_{1}^{c}\left(E_{0}, E_{1}\right)$ by conjugation. The universal property of a free crossed module holds since $F_{1}^{c}\left(E_{0}, E_{1}\right)$ is freely generated as a group by the conjugates,

$$
e_{1}^{c_{0}}=-c_{0}+e_{1}+c_{0}, \quad e_{1} \in E_{1}, c_{0} \in\left\langle E_{0}\right\rangle .
$$

Given two sets of relations $R_{i} \subset F_{i}^{c}\left(E_{0}, E_{1}\right), i=0,1$, the crossed module $C_{*}$ with generators $\left(E_{0}, E_{1}\right)$ and relations $\left(R_{0}, R_{1}\right)$ is defined as follows: $C_{0}$ is the quotient of $F_{0}^{c}\left(E_{0}, E_{1}\right)$ by the normal subgroup $N_{0}$ generated by $R_{0} \cup \partial R_{1}$, and $C_{1}$ is the quotient of $F_{1}^{c}\left(E_{0}, E_{1}\right)$ by the normal subgroup generated by,

$$
r_{1}^{c_{0}}, \quad r_{1} \in R_{1}, \quad c_{0} \in C_{0} ; \quad-c_{1}+c_{1}^{n_{0}}, \quad c_{1} \in C_{1}, n_{0} \in N_{0} .
$$

The action of $C_{0}$ on $C_{1}$ and the homomorphism $\partial: C_{1} \rightarrow C_{0}$ are defined so that the natural projection $F_{*}^{c}\left(E_{0}, E_{1}\right) \rightarrow C_{*}$ is a morphism of crossed modules.

Crossed modules defined by a presentation satisfy the obvious universal property. 
Definition 4.4.1. Given an $S_{\bullet}$-category $\mathscr{C}_{\bullet}$ we define $\mathcal{D}_{*}^{\prime}\left(\mathscr{C}_{\bullet}\right)$ as the crossed module presented by generators (G1)-(G3) and relations (R1)(R8) as in Definition 4.3.1.

This crossed module is 0 -free. Indeed, $\mathcal{D}_{0}^{\prime}\left(\mathscr{C}_{\bullet}\right)$ is the free group generated by the objects of $\mathscr{C}_{1}$ different from $s_{0}(*)$.

Universal non-commutative determinant functors are defined as in Definition 4.2.3, taking values in categorical groups instead.

Theorem 4.4.2. There exists a universal non-commutative determinant functor det: $\mathscr{C}_{\bullet} \rightarrow \Gamma \mathcal{D}_{*}^{\prime}\left(\mathscr{C}_{\bullet}\right)$ defined by

- $\operatorname{det}(X)=[X]$ for any object in $\mathscr{C}_{1}$,

- $\operatorname{det}\left(X \stackrel{\sim}{\rightarrow} X^{\prime}\right)=\left(\left[X^{\prime}\right],\left[X \stackrel{\sim}{\rightarrow} X^{\prime}\right]\right)$ for any weak equivalence in $\mathscr{C}_{1}$,

- $\operatorname{det}(\Delta)=\left(\left[d_{1} \Delta\right],[\Delta]\right)$ for any object in $\mathscr{C}_{2}$.

This non-commutative determinant functor is strict. Moreover, for any crossed module $C_{*}$, the functor

$$
\begin{aligned}
\operatorname{hom}\left(\mathcal{D}_{*}^{\prime}\left(\mathscr{C}_{\bullet}\right), C_{*}\right) & \longrightarrow \operatorname{Det}_{s}\left(\mathscr{C}_{\bullet}, \Gamma C_{*}\right) \\
\varphi & \longmapsto(\Gamma \varphi) \circ \operatorname{det}
\end{aligned}
$$

is an isomorphism of groupoids. Here the source is a morphism groupoid in the 2-category of crossed modules.

This theorem can be proved as Theorem 4.3.4, mutatis mutandis.

Proposition 4.4.3. There exists a unique map

$$
\langle\cdot, \cdot\rangle: \mathcal{D}_{*}^{\prime}\left(\mathscr{C}_{\bullet}\right) \times \mathcal{D}_{*}^{\prime}\left(\mathscr{C}_{\bullet}\right) \longrightarrow \mathcal{D}_{*}^{\prime}\left(\mathscr{C}_{\bullet}\right)
$$

such that

(1) $\langle[X],[Y]\rangle=-\left[s_{0} X \sqcup s_{1} Y\right]+\left[s_{1} X \sqcup s_{0} Y\right]$ for any two objects $X, Y$ in $\mathscr{C}_{1}$.

(2) $\left(\mathcal{D}_{*}^{\prime}\left(\mathscr{C}_{\bullet}\right),\langle\cdot, \cdot\rangle\right)$ is a reduced 2 -module.

Moreover, this map satisfies

$$
\langle a, b\rangle+\langle b, a\rangle=0
$$

for any $a, b \in \mathcal{D}_{0}^{\prime}\left(\mathscr{C}_{\bullet}\right)$, i.e. $\left(\mathcal{D}_{*}^{\prime}\left(\mathscr{C}_{\bullet}\right),\langle\cdot, \cdot\rangle\right)$ is a stable 2 -module.

Proof: We use the same argument as in [Wit1, Lemma 2.2.3]. The relations for the objects $s_{i} s_{j}(X)$ in $\mathscr{C}_{3}$ imply $\left\langle[X],\left[s_{0}(*)\right]\right\rangle=\left\langle\left[s_{0}(*)\right],[X]\right\rangle=0$ for any object in $\mathscr{C}_{1}$. Recall that $\left[s_{0}(*)\right]=0$. Since the group $\mathcal{D}_{0}^{\prime}\left(\mathscr{C}_{\bullet}\right)$ is the free group over the set $E$ of objects of $\mathscr{C}_{1}$ minus the degenerate object $s_{0}(*)$, an induction over the reduced word length of the two arguments shows that the map

$$
E \times E \longrightarrow \mathcal{D}_{1}^{\prime}\left(\mathscr{C}_{\bullet}\right), \quad(X, Y) \longmapsto-\left[s_{0} X \sqcup s_{1} Y\right]+\left[s_{1} X \sqcup s_{0} Y\right]
$$


extends in a unique way to a map

$$
\langle\cdot, \cdot\rangle: \mathcal{D}_{*}^{\prime}\left(\mathscr{C}_{\bullet}\right) \times \mathcal{D}_{*}^{\prime}\left(\mathscr{C}_{\bullet}\right) \longrightarrow \mathcal{D}_{*}^{\prime}\left(\mathscr{C}_{\bullet}\right)
$$

satisfying

(1) $\left\langle c, c^{\prime}+c^{\prime \prime}\right\rangle=\left\langle c, c^{\prime}\right\rangle^{c^{\prime \prime}}+\left\langle c, c^{\prime \prime}\right\rangle$,

(2) $\left\langle c+c^{\prime}, c^{\prime \prime}\right\rangle=\left\langle c^{\prime}, c^{\prime \prime}\right\rangle+\left\langle c, c^{\prime \prime}\right\rangle^{c^{\prime}}$.

It remains to show that $\left(\mathcal{D}_{*}^{\prime}\left(\mathscr{C}_{\bullet}\right),\langle\cdot, \cdot\rangle\right)$ is a stable 2 -module. For this, it suffices to check the axioms (3), (4), (6), and (8) in Subsection 3.2. Axioms (3) and (6) are immediate from the definition of $\langle\cdot, \cdot\rangle$.

We verify axiom (8). Let $X$ and $Y$ be objects of $\mathscr{C}_{1}$. Given two coproducts $s_{1} X \sqcup s_{0} Y$ and $s_{0} Y \sqcup s_{1} X$ their universal property yields a unique isomorphism fitting into the following commutative diagram,

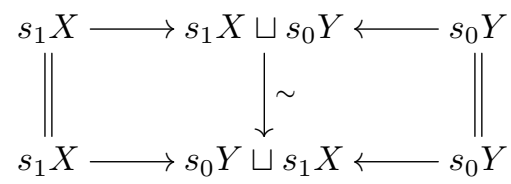

where the horizontal arrows are the inclusions of the factors. This isomorphism and the corresponding one after exchanging $X$ and $Y$ yield the following relations,

$$
\begin{gathered}
{\left[d_{2}\left(s_{1} X \sqcup s_{0} Y \stackrel{\sim}{\longrightarrow} s_{0} Y \sqcup s_{1} X\right)\right]+\left[d_{0}\left(s_{1} X \sqcup s_{0} Y \stackrel{\sim}{\longrightarrow} s_{0} Y \sqcup s_{1} X\right)\right]^{[X]}} \\
=-\left[s_{0} Y \sqcup s_{1} X\right]+\left[d_{1}\left(s_{1} X \sqcup s_{0} Y \stackrel{\sim}{\longrightarrow} s_{0} Y \sqcup s_{1} X\right)\right]+\left[s_{1} X \sqcup s_{0} Y\right], \\
{\left[d_{2}\left(s_{1} Y \sqcup s_{0} X \stackrel{\sim}{\longrightarrow} s_{0} X \sqcup s_{1} Y\right)\right]+\left[d_{0}\left(s_{1} Y \sqcup s_{0} X \stackrel{\sim}{\longrightarrow} s_{0} X \sqcup s_{1} Y\right)\right]^{[Y]}} \\
=-\left[s_{0} X \sqcup s_{1} Y\right]+\left[d_{1}\left(s_{1} Y \sqcup s_{0} X \stackrel{\sim}{\longrightarrow} s_{0} X \sqcup s_{1} Y\right)\right]+\left[s_{1} Y \sqcup s_{0} X\right] .
\end{gathered}
$$

Moreover, we have

$$
\begin{aligned}
& d_{i}\left(s_{1} X \sqcup s_{0} Y \stackrel{\sim}{\longrightarrow} s_{0} Y \sqcup s_{1} X\right)= \begin{cases}1_{Y}, & i=0 ; \\
X \sqcup Y \stackrel{\sim}{\longrightarrow} Y \sqcup X, & i=1 ; \\
1_{X}, & i=2 ;\end{cases} \\
& d_{i}\left(s_{1} Y \sqcup s_{0} X \stackrel{\sim}{\longrightarrow} s_{0} X \sqcup s_{1} Y\right)= \begin{cases}1_{X}, & i=0 ; \\
Y \sqcup X \stackrel{\sim}{\longrightarrow} X \sqcup Y, & i=1 ; \\
1_{Y}, & i=2 ;\end{cases}
\end{aligned}
$$

and hence,

$$
\begin{aligned}
\langle[X],[Y]\rangle+\langle[Y],[X]\rangle & =-([X \sqcup Y \stackrel{\sim}{\longrightarrow} Y \sqcup X]+[Y \sqcup X \stackrel{\sim}{\longrightarrow} X \sqcup Y])^{\partial\left[s_{1} Y \sqcup s_{0} X\right]} \\
& =-\left[1_{Y \sqcup X}\right]^{\partial\left[s_{1} Y \sqcup s_{0} X\right]}=0 .
\end{aligned}
$$


By induction it follows that $\left\langle c, c^{\prime}\right\rangle+\left\langle c^{\prime}, c\right\rangle=0$ for any pair of elements $c, c^{\prime}$ in $\mathcal{D}_{0}^{\prime}\left(\mathscr{C}_{\bullet}\right)$.

Finally, we verify axiom (4). Since both sides of the axiom define operations of $\mathcal{D}_{0}^{\prime}(\mathscr{C})$ on $\mathcal{D}_{1}^{\prime}(\mathscr{C})$, it suffices to check the relation for the action of an object $U$ of $\mathscr{C}_{1}$ on a weak equivalence $\alpha: X \rightarrow X^{\prime}$ in $\mathscr{C}_{1}$ and on an object $\Delta$ in $\mathscr{C}_{2}$, respectively. The weak equivalences $s_{1} \alpha \sqcup s_{0} 1_{U}$ and $s_{0} \alpha \sqcup s_{1} 1_{U}$ in $\mathscr{C}_{2}$ imply

$$
\begin{aligned}
{\left[s_{1} X^{\prime} \sqcup s_{0} U\right]+[\alpha] } & =\left[\alpha \sqcup 1_{U}\right]+\left[s_{1} X \sqcup s_{0} U\right], \\
{\left[s_{0} X^{\prime} \sqcup s_{1} U\right]+[\alpha]^{[U]} } & =\left[\alpha \sqcup 1_{U}\right]+\left[s_{0} X \sqcup s_{1} U\right],
\end{aligned}
$$

and hence,

$$
\begin{aligned}
{[\alpha]^{[U]} } & =\left\langle\left[X^{\prime}\right],[U]\right\rangle+[\alpha]+\langle[U],[X]\rangle \\
& =[\alpha]+\left\langle[U],-\left[X^{\prime}\right]\right\rangle^{[X]}+\langle[U],[X]\rangle=[\alpha]+\langle[U], \partial[\alpha]\rangle .
\end{aligned}
$$

The objects $s_{0}(\Delta) \sqcup s_{1} s_{1}(U), s_{1}(\Delta) \sqcup s_{0} s_{1}(U)$, and $s_{2}(\Delta) \sqcup s_{1} s_{0}(U)$ in $\mathscr{C}_{3}$ imply the relations

$$
\begin{aligned}
{\left[\Delta \sqcup s_{1} U\right]+\left[s_{0} d_{2} \Delta \sqcup s_{1} U\right] } & =\left[s_{0} d_{1} \Delta \sqcup s_{1} U\right]+[\Delta]^{[U]}, \\
{\left[\Delta \sqcup s_{1} U\right]+\left[s_{1} d_{2} \Delta \sqcup s_{0} U\right] } & =\left[\Delta \sqcup s_{0} U\right]+\left[s_{0} d_{0} \Delta \sqcup s_{1} U\right]^{\left[d_{2} \Delta\right]}, \\
{\left[s_{1} d_{1} \Delta \sqcup s_{0} U\right]+[\Delta] } & =\left[\Delta \sqcup s_{0} U\right]+\left[s_{1} d_{0} \Delta \sqcup s_{0} U\right]^{\left[d_{2} \Delta\right]} .
\end{aligned}
$$

From these, one deduces easily the relation

$$
[\Delta]^{[U]}=[\Delta]+\langle[U], \partial[\Delta]\rangle .
$$

Corollary 4.4.4. The morphism of stable 2-modules

$$
\begin{aligned}
\mathcal{D}_{*}^{\prime}\left(\mathscr{C}_{\bullet}\right) & \longrightarrow \mathcal{D}_{*}\left(\mathscr{C}_{\bullet}\right), \\
{[X] } & \longmapsto[X], \\
{\left[X \stackrel{\sim}{\longrightarrow} X^{\prime}\right] } & \longmapsto\left[X \stackrel{\sim}{\longrightarrow} X^{\prime}\right], \\
{[\Delta] } & \longmapsto[\Delta],
\end{aligned}
$$

is a quasi-isomorphism.

Proof: Let $C_{*}$ be a stable quadratic module. A morphism of stable 2-modules $\varphi: \mathcal{D}_{*}^{\prime}\left(\mathscr{C}_{\bullet}\right) \rightarrow C_{*}$ is the same as a morphism between the underlying crossed modules such that $\langle\varphi[X], \varphi[Y]\rangle=-\varphi\left[s_{0} X \sqcup s_{1} Y\right]+$ $\varphi\left[s_{1} X \sqcup s_{0} Y\right]$ for any two objects $X$ and $Y$ in $\mathscr{C}_{1}$. This, together with the crossed module presentation of $\mathcal{D}_{*}^{\prime}\left(\mathscr{C}_{\bullet}\right)$, shows that the morphism in the statement is well defined, and moreover, any morphism of stable 2-modules $\varphi: \mathcal{D}_{*}^{\prime}\left(\mathscr{C}_{\bullet}\right) \rightarrow C_{*}$ factors uniquely through the morphism in the statement. Therefore, the stable quadratic module obtained 
as the reflection of the stable 2 -module $\mathcal{D}_{*}^{\prime}\left(\mathscr{C}_{\bullet}\right)$ in the sense of [MT1, Lemma 4.18$]$ is $\mathcal{D}_{*}\left(\mathscr{C}_{\bullet}\right)$, and the unit of the reflection is the morphism in the statement. Hence this morphism is a quasi-isomorphism by [MT1, Remark 4.21].

Corollary 4.4.5. The determinant functor det: $\mathscr{C}_{\bullet} \rightarrow \Gamma \mathcal{D}_{*}\left(\mathscr{C}_{\bullet}\right)$ in Theorem 4.3 .4 is also universal among non-commutative determinant functors.

4.5. The connection with homotopy theory. In this section we consider the homotopy type of $\mid$ we $\mathscr{C}_{\bullet} \mid$, the geometric realization of the simplicial subcategory of weak equivalences in an $S_{\bullet}$-category $\mathscr{C}_{\bullet}$. This space is the geometric realization of a simplicial set: the diagonal of the bisimplicial nerve of we $\mathscr{C}_{\bullet}$. The bisimplicial nerve of a simplicial category is obtained by applying degreewise the nerve functor from categories to simplicial sets.

Notice that the space $\mid$ we $\mathscr{C}_{\bullet} \mid$ is connected. Moreover, it is reduced as a $C W$-complex, i.e. it has only one vertex, since we $\mathscr{C}_{0}=\mathscr{C}_{0}=*$ is the terminal category.

The categories $\mathscr{C}_{n}$ have finite coproducts, which are preserved by face and degeneracy operators, and finite coproducts of weak equivalences are weak equivalences. Hence, we can enhance $\mid$ we $\mathscr{C}_{\bullet} \mid$ to a $\Gamma$-space $A$ with $A(\mathbf{1})=\mid$ we $\mathscr{C}_{\bullet} \mid$, compare $[\mathbf{S e g}, \S 2]$. This $\Gamma$-space yields an $\Omega$-spectrum $K\left(\mathscr{C}_{\bullet}\right)$, with underlying sequence of spaces

$$
\Omega A(\mathbf{1}), A(\mathbf{1}), B A(\mathbf{1}), B^{2} A(\mathbf{1}), \ldots
$$

Indeed, it is enough to observe that $A(\mathbf{1})$ is the loop space of $B A(\mathbf{1})$, since $A(\mathbf{1})=\mid$ we $\mathscr{C}_{\bullet} \mid$ is connected, see [Seg, Proposition 1.4 and the following note].

Example 4.5.1. The spectra $K\left(\mathscr{C}_{\bullet}\right)$ of the $S_{\bullet}$-categories in Example 4.1.3 are:

(1) The $K$-theory spectrum $K(\mathscr{W})$ [Wal1] of a Waldhausen category $\mathscr{W}$.

(2) The derived $K$-theory spectrum of $\mathscr{W}, D K(\mathscr{W})$ [Gar1, Mur].

(4) Neeman's special $K$-theory spectrum $K\left({ }^{d} \mathscr{T}\right)$ of a triangulated category $\mathscr{T}[\mathbf{N e e 2}]$. This follows from the fact the inclusion of objects $S_{\bullet}\left({ }^{d} \mathscr{T}\right) \subset \operatorname{iso}\left(\bar{S}_{\bullet}\left({ }^{d} \mathscr{T}\right)\right)$ induces a homotopy equivalence on geometric realizations, compare [Wal2, corollary to Lemma 1.4.1].

(5) Neeman's virtual $K$-theory spectrum $K\left({ }^{v} \mathscr{T}\right)[\mathbf{N e e 2}]$, by the same reason as above. 
(6) Maltsiniotis's $K$-theory spectrum $K\left({ }^{s} \mathscr{T}_{\infty}\right)$ of a strongly triangulated category $\mathscr{T}_{\infty}[$ Mal1], again by the argument of the two previous cases.

(7) Neeman's graded $K$-theory spectrum $K\left(\mathrm{Gr}^{b} \mathscr{A}\right)$ of an abelian category $\mathscr{A}[\mathrm{Nee} 2]$.

(8) Garkusha's $K$-theory spectrum $K(\mathbb{D})$ of a right pointed derivator $\mathbb{D}[$ Gar2, §5], see also [Mal2].

In some of the previous references, the authors only care about the $K$-theory space of the corresponding $S_{\bullet}$-category $\mathscr{C}_{\bullet}$, i.e. $\Omega \mid$ we $\mathscr{C}_{\bullet} \mid$. We can always enhance them to spectra as indicated above.

Consider the functor $\lambda_{0}$ in Lemma 1.6.1.

Theorem 4.5.2. For any $S_{\bullet}$-category $\mathscr{C}_{\bullet}$, there is a natural isomorphism $\mathcal{D}_{*}\left(\mathscr{C}_{\bullet}\right) \cong \lambda_{0} K\left(\mathscr{C}_{\bullet}\right)$ in Hosquad.

This theorem is a straightforward generalization of [MT1, Theorem 1.7]. Exactly the same proof works with the appropriate changes in notation.

Example 4.5.3. The $S_{\bullet}$-functors in Example 4.1 .8 induce comparison maps of $K$-theory spectra:

(1) $K(\mathscr{W}) \rightarrow D K(\mathscr{W})$ for any Waldhausen category $\mathscr{W}[$ Gar1, Mur].

(2) $K\left({ }^{d} \mathscr{T}\right) \rightarrow K\left({ }^{v} \mathscr{T}\right)$ for any triangulated category $\mathscr{T}[\mathbf{N e e 2}$.

(3) $K(\mathscr{A}) \rightarrow K\left({ }^{d} \mathscr{T}\right)$ for any triangulated category $\mathscr{T}$ with a $t$-structure with heart $\mathscr{A}[\mathbf{N e e 2}]$.

(5) An equivalence $D K(\mathscr{W}) \stackrel{\sim}{\rightarrow} K\left(\mathbb{D}_{\mathscr{W}}\right)$ for any Waldhausen category $\mathscr{W}$ with cylinders satisfying the two-out-of-three axiom [Gar1, Mur].

(6) $K(\mathbb{D}) \rightarrow K\left({ }^{s} \mathbb{D}(*)\right)$ for any triangulated derivator $\mathbb{D}$ [Mal2].

(7) An equivalence $K(\mathscr{E}) \stackrel{\sim}{\rightarrow} K\left(C^{b}(\mathscr{E})\right)$ for any exact category $\mathscr{E}$ [Cis1].

(8) $K\left(C^{b}(\mathscr{E})\right) \rightarrow K\left({ }^{s} D^{b}(\mathscr{E})\right)$ for any exact category $\mathscr{E}$, compare [Mal1].

(9) An equivalence $K(\mathscr{A}) \stackrel{\sim}{\rightarrow} K\left(\mathrm{Gr}^{b} \mathscr{A}\right)$ for any abelian category $\mathscr{A}$ [Nee2].

Applying $\lambda_{0}$ to these maps we obtain some stable quadratic module morphisms described in Subsection 1.7. The equivalence (9) together with Theorems 4.3.4 and 4.5.2 shows that determinant functors on an abelian category $\mathscr{A}$ coincide essentially with graded determinant functors.

4.6. Generators and (some) relations for $\boldsymbol{\pi}_{\mathbf{1}}$. In this section we extend the results in [MT2] to the unified context introduced in this paper. We fix an $S_{\bullet}$-category $\mathscr{C}_{\bullet}$ satisfying the following additional property: 
- The functor sending an $(n+1)$-simplex to its $n+2$ faces,

$$
\phi: \mathscr{C}_{n+1} \longrightarrow \mathscr{C}_{n} \times \mathscr{C}_{n-1} \stackrel{n+2}{ }{ }^{+} \times \mathscr{C}_{n-1} \mathscr{C}_{n}, \quad n \geq 0,
$$

is a fibration of categories, i.e. it satisfies the isomorphism lifting property: any isomorphism $\phi(x) \rightarrow y$ in the target is the image by $\phi$ of an isomorphism $x \rightarrow x^{\prime}$ in the source, in particular $y=$ $\phi\left(x^{\prime}\right)$ is in the image of $\phi$.

This property is satisfied by Example 4.1.3(1), (3)-(7), but it need not be satisfied by Example 4.1.3(8) on derivators.

Definition 4.6.1. A triangle $\Delta$ in $\mathscr{C}_{\bullet}$ is just an object of $\mathscr{C}_{2}$. A weak triangle $(\Delta, f)$ in $\mathscr{C}_{\bullet}$ consists of a triangle $\Delta$ and a morphism $f: C \stackrel{\sim}{\rightarrow}$ $d_{0} \Delta$ in we $\left(\mathscr{C}_{1}\right)$. We denote,

$$
[\Delta, f]=[\Delta]+[f]^{\left[d_{2} \Delta\right]} \in \mathcal{D}_{1}\left(\mathscr{C}_{\bullet}\right) .
$$

A pair of triangles $\left(\Delta_{1} ; \Delta_{2}\right)$ consists of two triangles, $\Delta_{1}$ and $\Delta_{2}$, with the same faces $d_{i} \Delta_{1}=d_{i} \Delta_{2}, i=0,1,2$. A pair of triangles yields an element,

$$
\left[\Delta_{1} ; \Delta_{2}\right]=-\left[\Delta_{1}\right]+\left[\Delta_{2}\right] \in \pi_{1} \mathcal{D}_{*}\left(\mathscr{C}_{\bullet}\right) .
$$

A pair of weak triangles $\left(\Delta_{1}, f_{1} ; \Delta_{2}, f_{2}\right)$ consists of two weak triangles, $\left(\Delta_{1}, f_{1}\right)$ and $\left(\Delta_{2}, f_{2}\right)$, such that $\Delta_{1}$ and $\Delta_{2}$ have the same two last edges, $d_{1} \Delta_{1}=d_{1} \Delta_{2}, d_{2} \Delta_{1}=d_{2} \Delta_{2}$, and $f_{1}$ and $f_{2}$ have the same source,

$$
d_{0} \Delta_{1} \stackrel{f_{1}}{\longleftarrow} C \stackrel{f_{2}}{\longrightarrow} d_{0} \Delta_{2} .
$$

Any pair of weak triangles yields an element,

$$
\left[\Delta_{1}, f_{1} ; \Delta_{2}, f_{2}\right]=-\left[\Delta_{1}, f_{1}\right]+\left[\Delta_{2}, f_{2}\right] \in \pi_{1} \mathcal{D}_{*}\left(\mathscr{C}_{\bullet}\right) .
$$

(Pairs of) triangles are regarded as (pairs of) weak triangles, $\Delta=$ $\left(\Delta, 1_{d_{0} \Delta}\right)$ and $\left(\Delta_{1} ; \Delta_{2}\right)=\left(\Delta_{1}, 1_{d_{0} \Delta_{1}} ; \Delta_{2}, 1_{d_{0} \Delta_{2}}\right)$.

Notice that a trivial pair of weak triangles is trivial, i.e.

$$
[\Delta, f ; \Delta, f]=0 \in \pi_{1} \mathcal{D}_{*}\left(\mathscr{C}_{\bullet}\right) .
$$

Theorem 4.6.2. Any element in $\pi_{1} \mathcal{D}_{*}\left(\mathscr{C}_{\bullet}\right)$ is a pair of weak triangles.

We now extend to our unified framework enough results from [MT2] to ensure that the proof of [MT2, Theorem 2.1] generalizes to prove Theorem 4.6.2. 
A $3 \times 3$ diagram in an exact category $\mathscr{E}$ is a commutative diagram of short exact sequences,

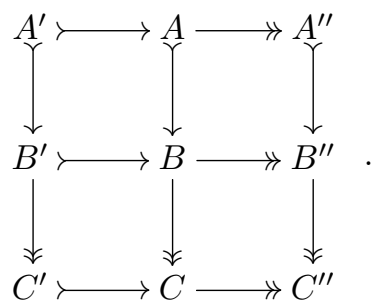

Such a diagram yields four objects in $S_{3} \mathscr{E}$,
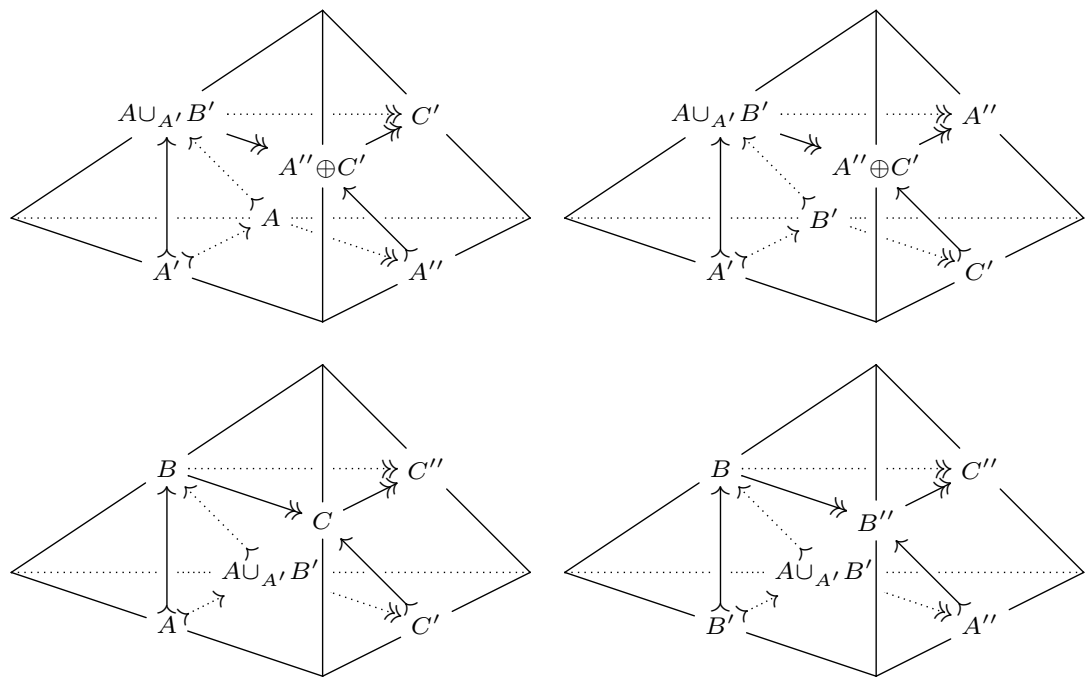

Based on this idea, we introduce the following notion of a $3 \times 3$ diagram in the $S_{\bullet}$-category $\mathscr{C}_{\bullet}$.

Definition 4.6.3. A $3 \times 3$ diagram in $\mathscr{C}_{\bullet}$ consists of four objects $\Theta_{1}$, $\Theta_{2}, \Theta_{3}, \Theta_{4}$ in $\mathscr{C}_{3}$ such that,

$$
\begin{aligned}
d_{2} \Theta_{1} & =d_{2} \Theta_{2}, & d_{1} \Theta_{3} & =d_{1} \Theta_{4}, \\
d_{1} \Theta_{1} & =d_{3} \Theta_{3}, & d_{1} \Theta_{2} & =d_{3} \Theta_{4}, \\
d_{0} d_{3} \Theta_{1} & =d_{0} d_{1} \Theta_{2}, & d_{0} d_{1} \Theta_{1} & =d_{0} d_{3} \Theta_{2}, \\
d_{0} \Theta_{1} & =s_{1} d_{0} d_{3} \Theta_{1} \sqcup s_{0} d_{0} d_{1} \Theta_{1}, & d_{0} \Theta_{2} & =s_{0} d_{0} d_{1} \Theta_{2} \sqcup s_{1} d_{0} d_{3} \Theta_{2} .
\end{aligned}
$$


The terminology ' $3 \times 3$ diagram' arises from:

Proposition 4.6.4. Given a $3 \times 3$ diagram in $\mathscr{C}_{\bullet}$ the following equation holds in $\mathcal{D}_{1}\left(\mathscr{C}_{\bullet}\right)$,

$$
\begin{aligned}
\left\langle\left[d_{0} d_{1} \Theta_{1}\right],\left[d_{0} d_{3} \Theta_{1}\right]\right\rangle= & -\left[d_{3} \Theta_{1}\right]-\left[d_{0} \Theta_{3}\right]^{\left[d_{2} d_{3} \Theta_{3}\right]}-\left[d_{2} \Theta_{3}\right] \\
& +\left[d_{2} \Theta_{4}\right]+\left[d_{0} \Theta_{4}\right]^{\left[d_{2} d_{3} \Theta_{4}\right]}+\left[d_{3} \Theta_{2}\right] .
\end{aligned}
$$

This result follows straightforwardly from (R8) and (R9).

Definition 4.6.5. A pair of $3 \times 3$ diagrams consists of two $3 \times 3$ diagrams, $\Theta_{1}, \Theta_{2}, \Theta_{3}, \Theta_{4}$ and $\Theta_{1}^{\prime}, \Theta_{2}^{\prime}, \Theta_{3}^{\prime}, \Theta_{4}^{\prime}$, such that for $i=0,1,2$,

$$
\begin{array}{lll}
d_{i} d_{3} \Theta_{1}=d_{i} d_{3} \Theta_{1}^{\prime}, & d_{i} d_{3} \Theta_{2}=d_{i} d_{3} \Theta_{2}^{\prime}, & d_{i} d_{0} \Theta_{3}=d_{i} d_{0} \Theta_{3}^{\prime}, \\
d_{i} d_{2} \Theta_{3}=d_{i} d_{2} \Theta_{3}^{\prime}, & d_{i} d_{0} \Theta_{4}=d_{i} d_{0} \Theta_{4}^{\prime}, & d_{i} d_{2} \Theta_{4}=d_{i} d_{2} \Theta_{4}^{\prime} .
\end{array}
$$

Corollary 4.6.6. For any pair of $3 \times 3$ diagrams in $\mathscr{C}_{\bullet}, \Theta_{1}, \Theta_{2}, \Theta_{3}$, $\Theta_{4}$ and $\Theta_{1}^{\prime}, \Theta_{2}^{\prime}, \Theta_{3}^{\prime}, \Theta_{4}^{\prime}$, the following relation between pairs of triangles in $\pi_{1} \mathcal{D}_{*}\left(\mathscr{C}_{\bullet}\right)$ holds,

$$
\begin{aligned}
& {\left[d_{3} \Theta_{1} ; d_{3} \Theta_{1}^{\prime}\right]-\left[d_{2} \Theta_{4} ; d_{2} \Theta_{4}^{\prime}\right]+\left[d_{0} \Theta_{3} ; d_{0} \Theta_{3}^{\prime}\right]} \\
& =\left[d_{3} \Theta_{2} ; d_{3} \Theta_{2}^{\prime}\right]-\left[d_{2} \Theta_{3} ; d_{2} \Theta_{3}^{\prime}\right]+\left[d_{0} \Theta_{4} ; d_{0} \Theta_{4}^{\prime}\right] .
\end{aligned}
$$

This is an easy consequence of Proposition 4.6.4, compare [MT2, Theorem 3.1].

Definition 4.6.7. A weak $3 \times 3$ diagram in $\mathscr{C}_{\bullet}$ consists of a $3 \times 3$ diagram $\Theta_{1}, \Theta_{2}, \Theta_{3}, \Theta_{4}$; two objects $\Delta_{1}, \Delta_{2}$ in $\mathscr{C}_{2}$ together with morphisms,

$$
w_{1}: \Delta_{1} \stackrel{\sim}{\longrightarrow} d_{0} \Theta_{3}, \quad w_{2}: \Delta_{2} \stackrel{\sim}{\longrightarrow} d_{0} \Theta_{4} ;
$$

and a commutative diagram in we $\left(\mathscr{C}_{1}\right)$,

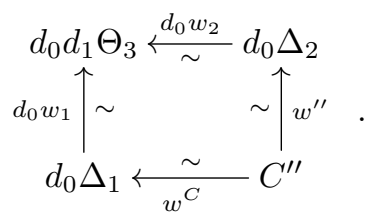

A pair of weak $3 \times 3$ diagrams consists of two weak $3 \times 3$ diagrams, the first one as before and the second one given by $\Theta_{1}^{\prime}, \Theta_{2}^{\prime}, \Theta_{3}^{\prime}, \Theta_{4}^{\prime}$,

$$
w_{1}^{\prime}: \Delta_{1}^{\prime} \stackrel{\sim}{\longrightarrow} d_{0} \Theta_{3}^{\prime}, \quad w_{2}^{\prime}: \Delta_{2}^{\prime} \stackrel{\sim}{\longrightarrow} d_{0} \Theta_{4}^{\prime} ; \quad \begin{gathered}
d_{0} d_{1} \Theta_{3}^{\prime} \stackrel{d_{0} w_{2}^{\prime}}{\sim} d_{0} \Delta_{2}^{\prime} \\
d_{1}^{\prime} \sim \underset{\sim}{\sim} \sim w^{\prime \prime \prime} \\
d_{0} \Delta_{1}^{\prime} \underset{\left(w^{C}\right)^{\prime}}{\sim} C^{\prime \prime}
\end{gathered}
$$


such that, for $i=1,2$,

$$
\begin{array}{ll}
d_{i} d_{3} \Theta_{1}=d_{i} d_{3} \Theta_{1}^{\prime}, & d_{i} d_{3} \Theta_{2}=d_{i} d_{3} \Theta_{2}^{\prime}, \\
d_{i} d_{2} \Theta_{4}=d_{i} d_{2} \Theta_{4}^{\prime}, & d_{i} d_{2} \Theta_{3}=d_{i} d_{2} \Theta_{3}^{\prime}, \\
\Delta_{1} & =d_{i} \Delta_{1}^{\prime},
\end{array}
$$

Proposition 4.6.8. Given a weak $3 \times 3$ diagram in $\mathscr{C}_{\bullet}$ as above, the following equation holds in $\mathcal{D}_{1}\left(\mathscr{C}_{\bullet}\right)$,

$$
\begin{aligned}
\left\langle\left[d_{2} \Delta_{1}\right],\left[d_{2} \Delta_{2}\right]\right\rangle= & -\left[d_{3} \Theta_{1}, d_{2} \Delta_{2}\right]-\left[\Delta_{1}, w^{C}\right]^{\left[d_{2} d_{3} \Theta_{3}\right]}-\left[d_{2} \Theta_{3}, d_{1} w_{1}\right] \\
& +\left[d_{2} \Theta_{4}, d_{1} w_{2}\right]+\left[\Delta_{2}, w^{\prime \prime}\right]^{\left[d_{2} d_{3} \Theta_{4}\right]}+\left[d_{3} \Theta_{2}, d_{2} w_{1}\right] .
\end{aligned}
$$

The proof of [MT2, Proposition 1.6] also works in this case.

Corollary 4.6.9. For any pair of weak $3 \times 3$ diagrams in $\mathscr{C}_{\bullet}$ as in the previous definition, the following relation between pairs of weak triangles in $\pi_{1} \mathcal{D}_{*}\left(\mathscr{C}_{\bullet}\right)$ holds,

$$
\begin{aligned}
& {\left[d_{3} \Theta_{1}, d_{2} \Delta_{2} ; d_{3} \Theta_{1}^{\prime}, d_{2} \Delta_{2}^{\prime}\right]-} {\left[d_{2} \Theta_{4}, d_{1} w_{2} ; d_{2} \Theta_{4}^{\prime}, d_{1} w_{2}^{\prime}\right] } \\
&+ {\left[\Delta_{1}, w^{C} ; \Delta_{1}^{\prime},\left(w^{C}\right)^{\prime}\right] } \\
&=\left[d_{3} \Theta_{2}, d_{2} w_{1} ; d_{3} \Theta_{2}^{\prime}, d_{2} w_{1}^{\prime}\right]-\left[d_{2} \Theta_{3}, d_{1} w_{1} ; d_{2} \Theta_{3}^{\prime}, d_{1} w_{1}^{\prime}\right] \\
&+\left[\Delta_{2}, w^{\prime \prime} ; \Delta_{2}^{\prime}, w^{\prime \prime \prime}\right] .
\end{aligned}
$$

This is an easy consequence of Proposition 4.6.8, compare [MT2, Theorem 3.1].

Corollary 4.6.10. Given two pairs of weak triangles in $\mathscr{C}_{\bullet},\left(\Delta_{1}, f_{1} ; \Delta_{1}^{\prime}, f_{1}^{\prime}\right)$ and $\left(\Delta_{2}, f_{2} ; \Delta_{2}^{\prime}, f_{2}^{\prime}\right)$, the following relation holds in $\pi_{1} \mathcal{D}_{*}\left(\mathscr{C}_{\bullet}\right)$,

$$
\left[\Delta_{1} \sqcup \Delta_{2}, f_{1} \sqcup f_{2} ; \Delta_{1}^{\prime} \sqcup \Delta_{2}^{\prime}, f_{1}^{\prime} \sqcup f_{2}^{\prime}\right]=\left[\Delta_{1}, f_{1} ; \Delta_{1}^{\prime}, f_{1}^{\prime}\right]+\left[\Delta_{2}, f_{2} ; \Delta_{2}^{\prime}, f_{2}^{\prime}\right] .
$$

Proof: Denote by $C$ the source of $f_{1}$ and $f_{1}^{\prime}$, and by $C^{\prime}$ the source of $f_{2}$ and $f_{2}^{\prime}$. This corollary follows by applying the previous one to the following pair of weak $3 \times 3$ diagrams:

$$
\begin{array}{ll}
\Theta_{1}^{i}=s_{0}^{2} d_{2} \Delta_{i} \sqcup s_{2} \Delta_{i}^{\prime}, & \Theta_{2}^{i}=s_{0} s_{1} d_{2} \Delta_{i} \sqcup s_{1} \Delta_{i}^{\prime}, \\
\Theta_{3}=s_{0} \Delta_{i} \sqcup s_{1}^{2} d_{1} \Delta_{i}^{\prime}, & \Theta_{4}=s_{1} \Delta_{i} \sqcup s_{2} \Delta_{i}^{\prime}, \\
\Delta_{1}^{i}=d_{0} \Theta_{3}^{i}, & w_{1}^{i}=1_{d_{0} \Theta_{3}^{i}}, \\
\Delta_{2}^{i}=s_{0} C \sqcup s_{1} C^{\prime}, & w_{2}=s_{0} f_{i} \sqcup s_{1} f_{i}^{\prime}, \\
w_{i}^{\prime \prime}=1_{C}, & w_{i}^{C}=f_{i} .
\end{array}
$$

The following result is completely new. It yields a smaller presentation of $\mathcal{D}_{*}\left(\mathscr{C}_{\bullet}\right)$ which can be applied in some important situations. 
Proposition 4.6.11. If weak equivalences in $\mathscr{C}_{\bullet}$ are isomorphisms, then $\mathcal{D}_{*}\left(\mathscr{C}_{\bullet}\right)$ has a presentation with generators $(\mathrm{G} 1)$ and (G3) and relations (R2), (R8), (R9), and $\left[s_{0}^{2}(*)\right]=0$.

Proof: This proof consists of an intensive use of the isomorphism lifting property in $\mathscr{C}_{\bullet}$ assumed at the beginning of this section. Any isomorphism $f: X \stackrel{\sim}{\rightarrow} X^{\prime}$ in $\mathscr{C}_{1}$ can be lifted to an isomorphism $\Phi(f): s_{1}(X) \stackrel{\sim}{\rightarrow}$ $\Delta(f)$ in $\mathscr{C}_{2}$ such that $d_{0} \Phi(f)$ is degenerate,

$$
d_{1} \Phi(f)=f, \quad d_{2} \Phi(f)=1_{X} .
$$

By (R7), $[f]=[\Delta(f)]$, therefore $\mathcal{D}_{*}(\mathscr{C})$ is generated by (G1) and (G3). By Remark 4.3.2, we now just have to check that (R6) and (R7) are redundant.

Given two composable isomorphisms in $\mathscr{C}_{1}$,

$$
X \stackrel{f}{\longrightarrow} Y \stackrel{g}{\longrightarrow} Z
$$

we can take an isomorphism $X i^{f, g}: s_{2} s_{1}(X) \stackrel{\sim}{\rightarrow} \Theta(f, g)$ in $\mathscr{C}_{3}$ such that, $d_{0} \Xi^{f, g}$ is degenerate,

$$
d_{1} \Xi^{f, g}=\Phi(g), \quad d_{2} \Xi^{f, g}=\Phi(g f), \quad d_{3} \Xi^{f, g}=\Phi(f) .
$$

If we apply (R8) to $\Theta(f, g)$ we obtain (R6).

Suppose now that $\Phi: \Delta_{1} \rightarrow \Delta_{2}$ is an isomorphism in $\mathscr{C}_{2}$. We choose two isomorphisms in $\mathscr{C}_{2}$,

$$
\Delta_{1} \stackrel{\Psi^{1}}{\longrightarrow} \Delta^{\prime} \stackrel{\Psi^{2}}{\longrightarrow} \Delta^{\prime \prime},
$$

with,

$$
\begin{array}{lll}
d_{0}\left(\Psi^{1}\right)=1_{d_{0} \Delta_{1}}, & d_{1}\left(\Psi^{1}\right)=d_{1} \Phi, & d_{2}\left(\Psi^{1}\right)=1_{d_{2} \Delta_{1}}, \\
d_{0}\left(\Psi^{2}\right)=d_{2} \Phi, & d_{1}\left(\Psi^{2}\right)=1_{d_{1} \Delta_{2}}, & d_{2}\left(\Psi^{2}\right)=1_{d_{2} \Delta_{1}},
\end{array}
$$

and two isomorphisms in $\mathscr{C}_{3}$,

$$
\Theta_{1}(\Phi) \stackrel{\Xi^{1}}{\longleftarrow} s_{1}(\Delta) \stackrel{\Xi^{2}}{\longrightarrow} \Theta_{2}(\Phi),
$$

with,

$$
\begin{aligned}
& d_{0} \Xi^{1}=1_{s_{1} d_{0} \Delta_{1}}, \quad d_{1} \Xi^{1}=\Phi\left(d_{1} \Phi\right), \quad d_{2} \Xi^{1}=\Psi^{1}, \quad d_{3} \Xi^{1}=1_{\Delta_{1}}, \\
& d_{0} \Xi^{2}=1_{d_{0} \Delta_{1}}, \quad d_{1} \Xi^{2}=\Phi, \quad d_{2} \Xi^{2}=\Psi^{2} \Psi^{1}, \quad d_{3} \Xi^{2}=\Phi\left(d_{2} \Phi\right) .
\end{aligned}
$$

We also consider $\Theta_{3}(\Phi)=s_{2} \Delta\left(d_{1} \Phi\right)$ and $\Theta_{4}(\Phi)=s_{2} \Delta_{2}$.

Now (R7) follows from Proposition 4.6.4 applied to the $3 \times 3$ diagram $\Theta_{1}(\Phi), \Theta_{2}(\Phi), \Theta_{3}(\Phi), \Theta_{4}(\Phi)$. Recall that Proposition 4.6.4 only uses (R8) and (R9), hence we are done. 
Definition 4.6.12. An $S_{\bullet}$-category $\mathscr{C}_{\bullet}$ has functorial coproducts if $\mathscr{C}_{n}$, $n \geq 0$, is endowed with a monoidal structure + , strictly compatible with face and degeneracy functors, which is strictly associative,

$$
(X+Y)+Z=X+(Y+Z),
$$

strictly unital with unit object $s_{0}^{n}(*)$,

$$
s_{0}^{n}(*)+X=X=X+s_{0}^{n}(*),
$$

and such that

$$
X=X+s_{0}^{n}(*) \longrightarrow X+Y \longleftarrow s_{0}^{n}(*)+Y=Y
$$

is always a coproduct diagram. Recall that $s_{0}^{n}(*)$ is an initial object in $\mathscr{C}_{n}, n \geq 0$.

We define the stable quadratic module $\mathcal{D}_{*}^{+}\left(\mathscr{C}_{\bullet}\right)$ as the quotient of $\mathcal{D}_{*}\left(\mathscr{C}_{\bullet}\right)$ by the following extra relation,

(R10) $\left[s_{0}(X)+s_{1}(Y)\right]=0$ for any pair of objects $X$ and $Y$ in $\mathscr{C}_{1}$.

Proposition 4.6.13. Let $\mathscr{C}_{\bullet}$ be an $S_{\bullet}$-category with functorial coproducts. If the set of objects of $\mathscr{C}_{1}$ is free as a monoid under + , then the natural projection,

$$
\mathcal{D}_{*}\left(\mathscr{C}_{\bullet}\right) \longrightarrow \mathcal{D}_{*}^{+}\left(\mathscr{C}_{\bullet}\right)
$$

is a weak equivalence. It actually forms part of a strong deformation retraction.

The proof is the same as the proof of [MT2, Theorem 4.2] with the obvious change of terminology. The hypothesis is not very strong.

Proposition 4.6.14. For any $S_{\bullet}$-category $\mathscr{C}_{\bullet}$ there is another one $\mathscr{C}_{\bullet}$ with functorial coproducts whose simplicial monoid of objects is freely generated by the simplicial set of objects in $\mathscr{C}_{\bullet} \bmod *$ and its degeneracies, and such that the natural simplicial functor $\mathscr{C}_{\bullet} \rightarrow \mathscr{C}_{\bullet}^{\prime}$ is an equivalence levelwise and restricts to a levelwise equivalence we $\left(\mathscr{C}_{\bullet}\right) \rightarrow \operatorname{we}\left(\mathscr{C}_{\bullet}^{\prime}\right)$.

For the proof of this proposition one applies levelwise the $\operatorname{Sum}(-)$ construction in [MT2, Proposition 4.3].

Lemma 4.6.15. Given two weak triangles $(\Delta, f)$ and $\left(\Delta^{\prime}, f^{\prime}\right)$ in an $S_{\bullet}-$ category with functorial coproducts $\mathscr{C}_{\bullet}$, if we denote $C$ and $C^{\prime}$ the source of $f$ and $f^{\prime}$, respectively, then the following relation holds in $\mathcal{D}_{1}^{+}\left(\mathscr{C}_{\bullet}\right)$,

$$
\left[\Delta+\Delta^{\prime}, f+f^{\prime}\right]=[\Delta, f]^{\left[d_{1} \Delta^{\prime}\right]}+\left[\Delta^{\prime}, f^{\prime}\right]+\left\langle\left[d_{2} \Delta\right],\left[C^{\prime}\right]\right\rangle .
$$


Proof: Apply Proposition 4.6.8 to

$$
\begin{array}{ll}
\Theta_{1}=s_{0}^{2} d_{2} \Delta+s_{2} \Delta^{\prime}, & \Theta_{2}=s_{0} s_{1} d_{2} \Delta+s_{1} \Delta^{\prime}, \\
\Theta_{3}=s_{0} \Delta+s_{1}^{2} d_{1} \Delta^{\prime}, & \Theta_{4}=s_{1} \Delta+s_{2} \Delta^{\prime}, \\
\Delta_{1}=d_{0} \Theta_{3}, & w_{1}=1_{d_{0} \Theta_{3}}, \\
\Delta_{2}=s_{0} C+s_{1} C^{\prime}, & w_{2}=s_{0} f+s_{1} f^{\prime}, \\
w^{\prime \prime}=1_{C}, & w^{C}=f .
\end{array}
$$

Corollary 4.6.16. Given two triangles $\Delta, \Delta^{\prime}$ in an $S_{\bullet}$-category with functorial coproducts $\mathscr{C}_{\bullet}$ and two weak equivalences $f: X \stackrel{\sim}{\rightarrow} Y, f^{\prime}: X^{\prime} \stackrel{\sim}{\rightarrow}$ $Y^{\prime}$ in $\mathscr{C}_{1}$, the following relations hold in $\mathcal{D}_{1}^{+}\left(\mathscr{C}_{\bullet}\right)$,

$$
\begin{aligned}
{\left[\Delta+\Delta^{\prime}\right] } & =[\Delta]^{\left[d_{1} \Delta^{\prime}\right]}+\left[\Delta^{\prime}\right]+\left\langle\left[d_{2} \Delta\right],\left[d_{0} \Delta^{\prime}\right]\right\rangle, \\
{\left[f+f^{\prime}\right] } & =[f]^{\left[Y^{\prime}\right]}+\left[f^{\prime}\right] .
\end{aligned}
$$

Lemma 4.6.17. Let $\mathscr{C}_{\bullet}$ be an $S_{\bullet}$-category with functorial coproducts, and $X_{1}, \ldots, X_{n}$ objects in $\mathscr{C}_{1}$. Given a permutation of $n$ elements, $\sigma \in$ $\operatorname{Sym}(n)$, we denote

$$
\sigma_{X_{1}, \ldots, X_{n}}: X_{\sigma_{1}}+\cdots+X_{\sigma_{n}} \longrightarrow X_{1}+\cdots X_{n}
$$

the isomorphism permuting the factors of the coproduct. The following formula holds in $\mathcal{D}_{1}^{+}(\mathscr{C})$,

$$
\left[\sigma_{X_{1}, \ldots, X_{n}}\right]=\sum_{\substack{i>j \\ \sigma_{i}<\sigma_{j}}}\left\langle\left[X_{\sigma_{i}}\right],\left[X_{\sigma_{j}}\right]\right\rangle .
$$

This lemma can be proved as [MT2, Lemma 4.9].

Proof of Theorem 4.6.2: In this proof we translate the argument in the proof of [MT2, Theorem 2.1] to our unified framework. By Proposition 4.6 .14 we can suppose that $\mathscr{C}_{\bullet}$ has functorial coproducts in such a way that the monoid of objects of $\mathscr{C}_{1}$ is freely generated by a set $\mathbb{S}$ of nondegenerate objects, so we can work with $\mathcal{D}_{1}^{+}\left(\mathscr{C}_{\bullet}\right)$ by Proposition 4.6.13.

Any $x \in \mathcal{D}_{1}^{+}\left(\mathscr{C}_{\bullet}\right)$ is a sum of triangles and weak equivalences in $\mathscr{C}_{1}$ with coefficients \pm 1 . Therefore, by Corollary 4.6.16, the following equation holds,

$$
\begin{aligned}
x= & -[f: X \stackrel{\sim}{\longrightarrow} Y]-[\Delta]+\left[\Delta^{\prime}\right]+\left[f^{\prime}: X^{\prime} \stackrel{\sim}{\longrightarrow} Y^{\prime}\right] & \bmod \langle\cdot, \cdot\rangle \\
= & -\left[f+1_{X^{\prime}}\right]-\left[\Delta+s_{0} d_{0} \Delta^{\prime}+s_{1} d_{2} \Delta^{\prime}\right] & \\
& +\left[s_{0} d_{0} \Delta+s_{1} d_{2} \Delta+\Delta^{\prime}\right]+\left[1_{X}+f^{\prime}\right] & \bmod \langle\cdot, \cdot\rangle .
\end{aligned}
$$


If $\partial(x)=0$ modulo commutators then,

$$
\begin{aligned}
0= & -\left[X+X^{\prime}\right]+\left[Y+X^{\prime}\right]-\left[d_{2} \Delta+d_{2} \Delta^{\prime}\right] \\
& -\left[d_{0} \Delta+d_{0} \Delta^{\prime}\right]+\left[d_{1} \Delta+d_{0} \Delta^{\prime}+d_{2} \Delta^{\prime}\right] \\
& -\left[d_{0} \Delta+d_{2} \Delta+d_{1} \Delta^{\prime}\right]+\left[d_{0} \Delta+d_{0} \Delta^{\prime}\right]+\left[d_{2} \Delta+d_{2} \Delta^{\prime}\right] \\
& -\left[X+Y^{\prime}\right]+\left[X+X^{\prime}\right]
\end{aligned}
$$

$\bmod [\cdot, \cdot]$

and therefore,

$\left[Y+X^{\prime}+d_{1} \Delta+d_{0} \Delta^{\prime}+d_{2} \Delta^{\prime}\right]=\left[X+Y^{\prime}+d_{0} \Delta+d_{2} \Delta+d_{1} \Delta^{\prime}\right] \quad \bmod [\cdot, \cdot]$.

The quotient of $\mathcal{D}_{0}^{+}\left(\mathscr{C}_{\bullet}\right)$ by the commutator subgroup is the free abelian group with basis $\mathbb{S}$, hence there are objects $S_{1}, \ldots, S_{n} \in \mathbb{S}$ and a permutation $\sigma \in \operatorname{Sym}(n)$ with,

$$
\begin{aligned}
Y+X^{\prime}+d_{1} \Delta+d_{0} \Delta^{\prime}+d_{2} \Delta^{\prime} & =S_{1}+\cdots+S_{n}, \\
X+Y^{\prime}+d_{0} \Delta+d_{2} \Delta+d_{1} \Delta^{\prime} & =S_{\sigma_{1}}+\cdots+S_{\sigma_{n}} .
\end{aligned}
$$

In particular, there is an isomorphism,

$$
\sigma_{S_{1}, \ldots, S_{n}}: X+Y^{\prime}+d_{0} \Delta+d_{2} \Delta+d_{1} \Delta^{\prime} \longrightarrow Y+X^{\prime}+d_{1} \Delta+d_{0} \Delta^{\prime}+d_{2} \Delta^{\prime} .
$$

By the isomorphism lifting property, there exists an isomorphism in $\mathscr{C}_{2}$,

$$
\Phi: s_{0} X+s_{0} Y^{\prime}+s_{0} d_{0} \Delta+s_{1} d_{2} \Delta+\Delta^{\prime} \stackrel{\sim}{\longrightarrow} \Delta_{2},
$$

such that $d_{0} \Phi$ and $d_{2} \Phi$ are identity morphisms and $d_{1} \Phi=\sigma_{S_{1}, \ldots, S_{n}}$.

By Corollaries 4.6.16 and 4.6.17, modulo the image of $\langle\cdot, \cdot\rangle$,

$$
\begin{aligned}
& x=-\left[f+1_{X^{\prime}}+1_{d_{0} \Delta+d_{0} \Delta^{\prime}}\right]-\left[s_{0} Y+s_{0} X^{\prime}+\Delta+s_{0} d_{0} \Delta^{\prime}+s_{1} d_{2} \Delta^{\prime}\right] \\
& +\left[s_{0} X+s_{0} Y^{\prime}+s_{0} d_{0} \Delta+s_{1} d_{2} \Delta+\Delta^{\prime}\right]+\left[1_{X}+f^{\prime}+1_{d_{0} \Delta+d_{0} \Delta^{\prime}}\right] \\
& =-\left[f+1_{X^{\prime}}+1_{d_{0} \Delta+d_{0} \Delta^{\prime}}\right]-\left[s_{0} Y+s_{0} X^{\prime}+\Delta+s_{0} d_{0} \Delta^{\prime}+s_{1} d_{2} \Delta^{\prime}\right] \\
& +\left[\sigma_{S_{1}, \ldots, S_{n}}\right] \\
& +\left[s_{0} X+s_{0} Y^{\prime}+s_{0} d_{0} \Delta+s_{1} d_{2} \Delta+\Delta^{\prime}\right]+\left[1_{X}+f^{\prime}+1_{d_{0} \Delta+d_{0} \Delta^{\prime}}\right] \\
& =-\left[f+1_{X^{\prime}}+1_{d_{0} \Delta+d_{0} \Delta^{\prime}}\right]-\left[s_{0} Y+s_{0} X^{\prime}+\Delta+s_{0} d_{0} \Delta^{\prime}+s_{1} d_{2} \Delta^{\prime}\right] \\
& +\left[\Delta_{2}\right]+\left[1_{X}+f^{\prime}+1_{d_{0} \Delta+d_{0} \Delta^{\prime}}\right] \\
& =-\left[f+1_{X^{\prime}}+1_{d_{0} \Delta+d_{0} \Delta^{\prime}}\right]^{\left[d_{2} \Delta+d_{2} \Delta^{\prime}\right]} \\
& -\left[s_{0} Y+s_{0} X^{\prime}+\Delta+s_{0} d_{0} \Delta^{\prime}+s_{1} d_{2} \Delta^{\prime}\right] \\
& +\left[\Delta_{2}\right]+\left[1_{X}+f^{\prime}+1_{d_{0} \Delta+d_{0} \Delta^{\prime}}\right]^{\left[d_{2} \Delta+d_{2} \Delta^{\prime}\right]} \\
& =\left[s_{0} Y+s_{0} X^{\prime}+\Delta+s_{0} d_{0} \Delta^{\prime}+s_{1} d_{2} \Delta^{\prime}, f+1_{X^{\prime}}+1_{d_{0} \Delta+d_{0} \Delta^{\prime}}\right. \text {; } \\
& \left.\Delta_{2}, 1_{X}+f^{\prime}+1_{d_{0} \Delta+d_{0} \Delta^{\prime}}\right],
\end{aligned}
$$


i.e. $x$ is represented by a pair of weak triangles modulo the image of $\langle\cdot, \cdot\rangle$,

$$
x=\left[\Delta_{1}, f_{1} ; \Delta_{2}, f_{2}\right]+y, \quad y \text { in the image of }\langle\cdot, \cdot\rangle .
$$

Assume now that $\partial(x)=0$. Then $\partial(y)=0$ as well, therefore by [MT2, Lemma 5.1] $y=\langle a, a\rangle$ for some $a \in \mathcal{D}_{0}\left(\mathscr{C}_{\bullet}\right)$, which is the free group of nilpotency class 2 with basis $\mathbb{S}$. Since $y$ only depends on $a$ mod 2 , we can suppose that $a=\left[S_{1}^{\prime}\right]+\cdots+\left[S_{m}^{\prime}\right]=[M], M=S_{1}^{\prime}+\cdots+S_{m}^{\prime}$, $S_{i}^{\prime} \in \mathbb{S}$, therefore,

$$
y=\langle[M],[M]\rangle=\left[s_{0} M+s_{1} M ; s_{1} M+s_{0} M\right],
$$

is a pair of triangles, in particular a pair of weak triangles, so $x$ is also a pair of weak triangles by Corollary 4.6.10.

\section{References}

[Bau] H. J. BAues, "Combinatorial homotopy and 4-dimensional complexes", With a preface by Ronald Brown, de Gruyter Expositions in Mathematics 2, Walter de Gruyter \& Co., Berlin, 1991. DOI: $10.1515 / 9783110854480$.

[BM] H.-J. Baues And F. Muro, Secondary homotopy groups, Forum Math. 20(4) (2008), 631-677. DOI: 10.1515/FORUM. 2008. 032.

[BBD] A. A. BeǏlinson, J. Bernstein, And P. Deligne, Faisceaux pervers, in: "Analysis and topology on singular spaces, I" (Luminy, 1981), Astérisque 100, Soc. Math. France, Paris, 1982, pp. $5-171$.

[Boy] M. Boyarchenko, Picard groupoids and spectra, Seminar notes, http://www.math.uchicago.edu/ ${ }^{\text {mitya. }}$

[Bre1] M. Breuning, Determinant functors on triangulated categories, J. K-Theory 8(2) (2011), 251-291. DOI: 10.1017/ is010006009jkt120.

[Bre2] M. Breuning, Determinants of perfect complexes and Euler characteristics in relative $K_{0}$-groups, Preprint (2008), arXiv:0812.1556v1.

[BS] R. Brown and C. B. Spencer, $G$-groupoids, crossed modules and the fundamental groupoid of a topological group, Nederl. Akad. Wetensch. Proc. Ser. A $79=$ Indag. Math. 38(4) (1976), 296-302.

[Cis1] D.-C. Cisinski, Théorèmes de cofinalité en $K$-théorie (d'après Thomason), Seminar notes (2002), http://www.math.univtoulouse.fr $/ \sim$ dcisinsk. 
[Cis2] D.-C. Cisinski, Catégories dérivables, Bull. Soc. Math. France 138(3) (2010), 317-393.

[Del] P. Deligne, Le déterminant de la cohomologie, in: "Current trends in arithmetical algebraic geometry" (Arcata, Calif., 1985), Contemp. Math. 67, Amer. Math. Soc., Providence, RI, 1987, pp. 93-177. DOI : 10.1090/conm/067/902592.

[Eps] D. B. A. Epstein, Functors between tensored categories, Invent. Math. 1 (1966), 221-228. DOI: 10.1007/BF01452242.

[Eri] D. ERIKsson, A Deligne-Riemann-Roch isomorphism I: Preliminaries on virtual categories, Preprint (2009), arXiv: 0904.4059v1.

[Fer] D. FERRAND, On the non additivity of the trace in derived categories, Preprint (2005), arXiv:math/0506589.

[FK] T. FukAYA AND K. KATO, A formulation of conjectures on $p$ adic zeta functions in noncommutative Iwasawa theory, in: "Proceedings of the St. Petersburg Mathematical Society", Vol. XII, Amer. Math. Soc. Transl. Ser. 2 219, Amer. Math. Soc., Providence, RI, 2006, pp. 1-85.

[Gar1] G. Garkusha, Systems of diagram categories and K-theory. II, Math. Z. 249(3) (2005), 641-682. DOI: 10.1007/s00209-0040726-0.

[Gar2] G. Garkusha, Systems of diagram categories and $K$-theory. I, (Russian), Algebra $i$ Analiz 18(6) (2006), 131-186; translation in St. Petersburg Math. J. 18(6) (2007), 957-996. DOI : 10.1090/ S1061-0022-07-00978-8.

[GM] S. I. Gelfand And Yuri I. Manin, "Methods of Homological Algebra", Second edition, Springer Monographs in Mathematics, Springer-Verlag, Berlin, 2003. DOI : 10.1007/978-3-662-12492-5.

[JS] A. Joyal And R. Street, Braided tensor categories, $A d v$. Math. 102(1) (1993), 20-78. DOI: 10.1006/aima.1993.1055.

[Kel] B. Keller, Appendice: Le dérivateur triangulé associé à une catégorie exacte, in: "Categories in algebra, geometry and mathematical physics", Contemp. Math. 431, Amer. Math. Soc., Providence, RI, 2007, pp. 369-373. DOI: 10.1090/conm/431/ 08355.

[KN] B. Keller And A. Neeman, The connection between May's axioms for a triangulated tensor product and Happel's description of the derived category of the quiver $D_{4}$, Doc. Math. 7 (2002), 535-560 (electronic).

[Knu1] F. F. KnUDsen, Determinant functors on exact categories and their extensions to categories of bounded complexes, Michigan Math. J. 50(2) (2002), 407-444. DOI: 10.1307/mmj/1028575741. 
[Knu2] F. F. Knudsen, Erratum: "Determinant functors on exact categories and their extensions to categories of bounded complexes" [Michigan Math. J. 50(2) (2002), 407-444] by F. F. Knudsen, Michigan Math. J. 50(3) (2002), 665.

[KM] F. F. KNUDSEn AND D. MumFord, The projectivity of the moduli space of stable curves. I. Preliminaries on "det" and "Div", Math. Scand. 39(1) (1976), 19-55.

[Kün] M. KünZER, Nonisomorphic Verdier octahedra on the same base, J. Homotopy Relat. Struct. 4(1) (2009), 7-38.

[Lap] M. L. LAPLAZA, Coherence for categories with group structure: an alternative approach, J. Algebra 84(2) (1983), 305-323. DOI: 10.1016/0021-8693(83)90081-9.

[Mac1] S. MACLANE, Cohomology theory in abstract groups. III. Operator homomorphisms of kernels, Ann. of Math. (2) 50 (1949), 736-761. DOI: 10.2307/1969561.

[Mac2] S. MACLANE, "Categories for the working mathematician", Graduate Texts in Mathematics 5, Springer-Verlag, New YorkBerlin, 1971.

[Mal1] G. Maltsiniotis, Catégories triangulées supérieures, Preprint (2006), http://www.math.jussieu.fr/〜maltsin.

[Mal2] G. Maltsiniotis, La $K$-théorie d'un dérivateur triangulé, in: "Categories in algebra, geometry and mathematical physics", Contemp. Math. 431, Amer. Math. Soc., Providence, RI, 2007, pp. 341-368. DOI : 10.1090/conm/431/08280.

[Mur] F. Muro, Maltsiniotis's first conjecture for $K_{1}$, Int. Math. Res. Not. IMRN 2008(4) (2008), 31 pp.

[MSS] F. Muro, S. Schwede, And N. Strickland, Triangulated categories without models, Invent. Math. 170(2) (2007), 231-241. DOI: 10.1007/s00222-007-0061-2.

[MT1] F. Muro And A. Tonks, The 1-type of a Waldhausen $K$ theory spectrum, Adv. Math. 216(1) (2007), 178-211. DOI: 10.1016/j . aim.2007.05.008.

[MT2] F. Muro AND A. TONKS, On $K_{1}$ of a Waldhausen category, in: "K-theory and noncommutative geometry", EMS Ser. Congr. Rep., Eur. Math. Soc., Zürich, 2008, pp. 91-115. DOI: 10.4171/ 060-1/4.

[Nee1] A. NeEman, "Triangulated categories", Annals of Mathematics Studies 148, Princeton University Press, Princeton, NJ, 2001.

[Nee2] A. NeEman, The $K$-theory of triangulated categories, in: "Handbook of K-theory", Vol. 1, 2, Springer, Berlin, 2005, pp. 1011-1078. DOI : 10.1007/978-3-540-27855-9_20. 
[Nen] A. Nenashev, $K_{1}$ by generators and relations, J. Pure Appl. Algebra 131(2) (1998), 195-212. DOI: 10.1016/S0022-4049(97) 00056-X.

[Qui] D. Quillen, Higher algebraic $K$-theory. I, in: "Algebraic K-theory, I: Higher K-theories "(Proc. Conf., Battelle Memorial Inst., Seattle, Wash., 1972), Lecture Notes in Math. 341, Springer, Berlin, 1973, pp. 85-147.

[Ran] A. RANicki, The algebraic theory of torsion. I. Foundations, in: "Algebraic and geometric topology" (New Brunswick, N.J., 1983), Lecture Notes in Math. 1126, Springer, Berlin, 1985, pp. 199-237. DOI : 10.1007/BFb0074445.

[Sch] M. Schlichting, A note on $K$-theory and triangulated categories, Invent. Math. 150(1) (2002), 111-116. DOI: 10.1007/ s00222-002-0231-1.

[Seg] G. Segal, Categories and cohomology theories, Topology 13 (1974), 293-312.

[Sin] H. X. Sinh, Gr-catégories, Ph.D. Thesis, Université Paris 7 (1975).

[Vak1] A. VAKnin, Determinants in triangulated categories, $K$-Theory 24(1) (2001), 57-68. DOI: 10.1023/A:1012284502759.

[Vak2] A. VAKnIN, $K_{1}$ for triangulated categories, $K$-Theory 24(1) (2001), 1-56. DOI: 10.1023/A:1012244700846.

[Vak3] A. VAKnin, Virtual triangles, K-Theory 22(1-2) (2001), 161198. DOI: $10.1023 / \mathrm{A}: 1011109407704$.

[Wal1] F. Waldhausen, Algebraic $K$-theory of topological spaces. I, in: "Algebraic and geometric topology" (Proc. Sympos. Pure Math., Stanford Univ., Stanford, Calif., 1976), Part 1, Proc. Sympos. Pure Math. XXXII, Amer. Math. Soc., Providence, R.I., 1978, pp. 35-60.

[Wal2] F. Waldhausen, Algebraic $K$-theory of spaces, in: "Algebraic and geometric topology" (New Brunswick, N.J., 1983), Lecture Notes in Math. 1126, Springer, Berlin, 1985, pp. 318-419. DOI: 10.1007/BFb0074449.

[Wei] M. WeIss, Hammock localization in Waldhausen categories, $J$. Pure Appl. Algebra 138(2) (1999), 185-195. DOI: 10.1016/ S0022-4049 (98) 00009-7.

[Wit1] M. WitTe, Noncommutative Iwasawa main conjectures for varieties over finite fields, Ph.D. Thesis, Fakultät für Mathematik und Informatik der Universität Leipzig (2008), http://d-nb.info/995008124. 
[Wit2] M. WiTte, On a noncommutative Iwasawa main conjecture for varieties over finite fields, J. Eur. Math. Soc. (JEMS) 16(2) (2014), 289-325. DOI : 10.4171/JEMS/434.

Fernando Muro:

Universidad de Sevilla

Facultad de Matemáticas

Departamento de Álgebra

Avda. Reina Mercedes s/n

41012 Sevilla

Spain

E-mail address: fmuro@us.es

Andrew Tonks:

Department of Mathematics

University of Leicester

University Road

Leicester LE1 7RH

United Kingdom

E-mail address: a.tonks@gmail.com

Malte Witte:

Ruprecht-Karls-Universität Heidelberg

Mathematisches Institut

Im Neuenheimer Feld 288

69120 Heidelberg

Germany

E-mail address: witte@mathi.uni-heidelberg.de

Primera versió rebuda el 15 d'octubre de 2013, darrera versió rebuda el 4 d'abril de 2014. 